\title{
Solving direct and inverse heat conduction problems in functionally graded materials using an accurate and robust numerical method
}

\author{
Farzad Mohebbi ${ }^{*}$, Ben Evans ${ }^{1}$, Timon Rabczuk ${ }^{2}$ \\ ${ }^{1}$ Zienkiewicz Centre for Computational Engineering, College of Engineering, Swansea \\ University, Bay Campus, Fabian Way, Crymlyn Burrows, Swansea SA1 8EN, UK \\ ${ }^{2}$ Institute of Structural Mechanics, Bauhaus-University Weimar, Marienstraße 1599423 Weimar, \\ Germany
}

\begin{abstract}
In this study we present a numerical approach to solve steady-state heat conduction problems in functionally graded materials (FGMs). Two different types of material gradations are considered for spatially varying thermal conductivity of FGM: (1) Quadratic material gradation; (2) Exponential material gradation. The proposed numerical procedure is based on finite-difference method and is developed to solve the steady-state heat conduction equation over a general two-dimensional (irregular) heat conducting body (FGM) with Dirichlet, Neumann, and Robin boundary conditions. In addition to presenting an accurate heat conduction equation solution considering an irregular shape and a variety of boundary conditions, the other novel aspect of this study is to identify the constant parameters in the material gradations accurately by an inverse analysis thereby determining the accurate form of gradation. The novelty of the inverse analysis lies in proposing an accurate and efficient explicit sensitivity analysis scheme. The main advantage of the sensitivity analysis scheme is that it is not involved with an adjoint equation and all the sensitivity coefficients can be explicitly computed in only one direct solution. The conjugate gradient method (CGM) is used to reduce the mismatch between the computed temperature on part of the boundary and the simulated measured temperature distribution. The accuracy, efficiency, and robustness of the proposed numerical approach are demonstrated through presenting two test cases.
\end{abstract}

\footnotetext{
* Corresponding author

Email address: farzad.mohebbi@swansea.ac.uk, farzadmohebbi@yahoo.com
} 


\section{Introduction}

Functionally graded materials (FGMs), initially proposed to be a solution for structures withstanding high surface temperatures in aerospace industry, are a relatively new class of composite materials with attractive thermo-mechanical properties and extensive uses in engineering applications subjected to, say, high temperature and high thermal stress arising in aerospace, pressure vessel, and nuclear reactors, to name a few. These materials are inhomogeneous composites which are composed of two or more constituents phases. By gradually varying the volume fraction of constituent materials of FGMs, their properties (such as thermal conductivity, modulus of elasticity, density, etc) vary smoothly and continuously with position [1-3]. Due to the ever-increasing importance of thermo-mechanical behavior of FGMs in engineering applications, these materials have been subject of intensive research during past decades and many analytical and computational methods are adopted to model and analyze such a behavior under different thermo-mechanical loading conditions.

In [4], an exact analytical solution based on Ferrari's method is derived for steady-state heat transfer in functionally graded sandwich slabs under convective-radiative boundary conditions. In [5] Green's Functions and boundary integral analysis is employed to obtain closed-form expressions for two and three-dimensional heat conduction equation in FGMs considering an exponential thermal conductivity gradation. In [6], a boundary element method is presented to solve transient heat conduction in FGMs with quadratic, exponential, and trigonometric gradation of thermal conductivity and specific heat. In [7], a numerical framework based on combination of generalized finite-difference method and Krylov deferred correction is presented to solve 3D transient heat conduction problems in FGMs. Four types of material gradations, namely, quadratic, exponential, logarithmic, and trigonometric, are considered for thermal conductivity gradation in the solid body.

Accurate determination of temperature distribution over heat conducting bodies requires that the thermo-physical properties of material and associated boundary conditions are precisely known. However, accurate knowledge of such quantities involves expensive experiments with sophisticated instruments. Hence, inverse methods, as inexpensive alternatives, may be employed to estimate the thermo-physical properties such as the 
thermal conductivity (constant, temperature-dependent, and spatially varying parameter) and the convection heat transfer coefficient [8-38], the heat flux [39-43], and the boundary shape of bodies [44-48] using temperature measurement taken at some points inside the body or on some part of the boundary. However, due to ill-posed nature, Inverse Heat Transfer Problems (IHTPs) are mathematically challenging problems because the ill-posed problems are inherently unstable and very sensitive to noise and special methods are required to solve these problems [49-51]. Due to the power of high-speed computers, the numerical treatment of IHTP in FGMs has also received much attention among engineers and mathematicians. In [52], Cauchy problem for steady-state heat conduction in two-dimensional anisotropic FGMs are addressed. The method of fundamental solution is employed to solve this inverse problem and since the obtained system of equations is ill-conditioned, the Tikhonov regularization method is employed to solve it. In [53], an inverse method is used to estimate the unknown spacedependent thermal conductivity of a functionally graded hollow cylinder. The method is based on the conjugate gradient method and the discrepancy principle and the success of the inverse method depends on the type of the boundary conditions. In [54], spatially varying properties of solid material such as thermal conductivity is determined using temperature measurement taken on the boundary of solid material. In [55], an inverse method based on the conjugate gradient method and the discrepancy principle is employed to simultaneously estimate the unknown time-dependent inner and outer boundary heat fluxes in a functionally graded hollow circular cylinder from the knowledge of temperature measurements taken within the cylinder. Then distribution of temperature and thermal stresses in the hollow cylinder is determined. In [56], an inverse analysis based on the conjugate gradient method and the discrepancy principle is employed to estimate the time and spatially varying pressure and heat flux in functionally graded cylindrical shells with finite length by using the measured displacements and temperatures on their outer surfaces. The governing equations of the direct, adjoint, and sensitivity problems under related boundary and initial conditions are solved by using differential quadrature method (as a numerical tool to analyze different problems of functionally graded shells). 
In the literature, however, there still exist some limitations on the methods proposed by different researchers to address spatially varying thermal conductivity. Some of these limitations can be summarized as follows:

- The heat conduction problem is concerned with regular bodies only (inability to address the irregular bodies) and the heat conduction equation is solved using the traditional finite-difference method.

- Most of the boundary conditions in the literature are either a constant temperature (Dirichlet boundary condition) or insulated case without addressing the Neumann, and Robin types.

- Many of the proposed methods to address the spatially variation of thermal conductivity are limited to one-dimensional problems.

Thus a general methodology for a general 2D domain and boundary conditions considering a spatially varying thermal conductivity as a FGM property with a high degree of accuracy is required. This paper deals with two-dimensional steady-state heat conduction problems in FGMs in which the thermal conductivity of FGM varies quadratically and exponentially with position in $x$ - and $y$ - directions, that is, $k=f(x, y)$. In addition to presenting an accurate heat conduction equation solution considering an irregular shape and a variety of boundary conditions, the other novel aspect of this study is to identify the constant parameters in the thermal conductivity gradations accurately by an inverse analysis thereby determining the accurate form of the gradation. To do so, elliptic grid generation technique is used to generate a mesh over the irregular body and then solve for the steady-state heat conduction equation by transforming the body shape (physical domain), the governing equation and the associated boundary conditions onto the computational domain. The discretization in the computational domain is based on the finite-difference method, a method chosen for its simplicity and ease of implementation. The most innovative aspect of the numerical approach is its very efficient and accurate sensitivity analysis scheme in which explicit expressions for the sensitivity coefficients are derived which allow for the computation of all sensitivity coefficients in one single solve only. The conjugate gradient method is employed to minimize the difference between the computed temperature on part of the boundary and the simulated measured temperature. 


\section{Governing equation}

The mathematical representation of the steady-state heat conduction problem of interest here can be expressed as below (see Fig. 1)

$$
\frac{\partial}{\partial x}\left(k(x, y) \frac{\partial T}{\partial x}\right)+\frac{\partial}{\partial y}\left(k(x, y) \frac{\partial T}{\partial y}\right)=0 \text { in physical domain } \Omega
$$

subject to the boundary conditions

$$
\begin{gathered}
\frac{\partial T}{\partial n}=\frac{\dot{q}}{k} \text { on boundary surface } \Gamma_{1} \\
\frac{\partial T}{\partial n}=-\frac{h_{i}}{k}\left(T_{\Gamma_{i}}-T_{\infty_{i}}\right) \text { on boundary surface } \Gamma_{i}, i=2,3,4
\end{gathered}
$$

Two different types are considered for the spatially varying thermal conductivity (material gradation type) which will be considered separately:

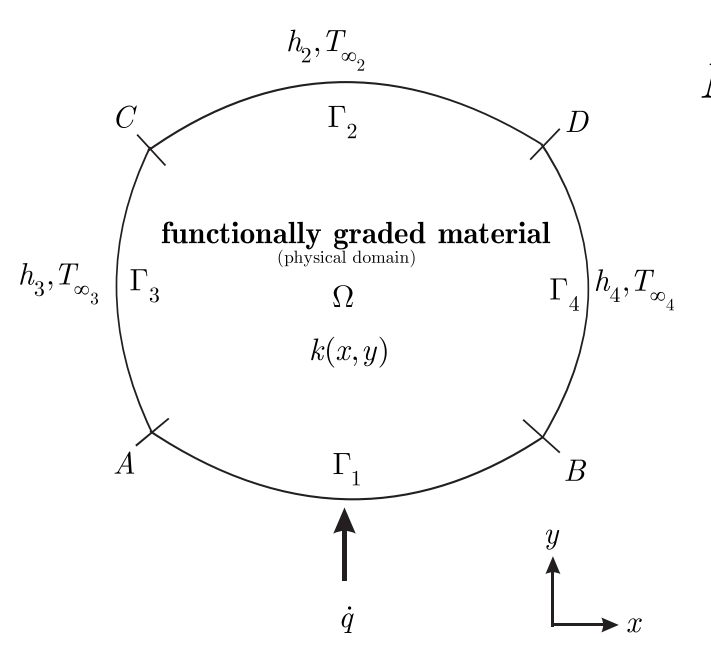

a)

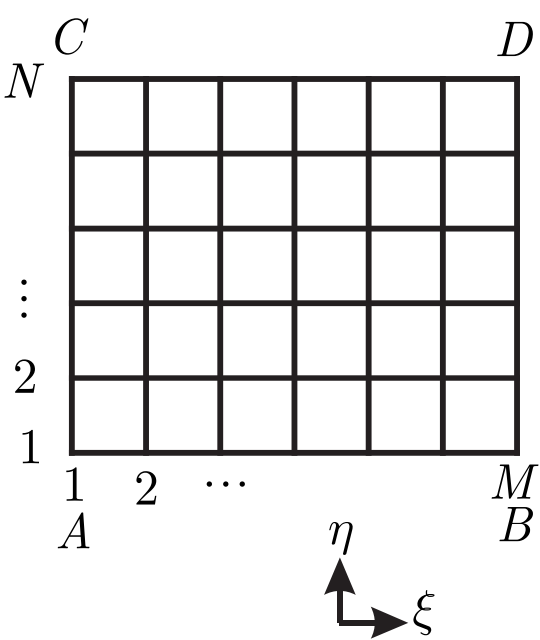

b)

Fig. 1 Arbitrarily shaped two-dimensional heat-conducting body (physical domain) subjected to convective heat transfer on surfaces $\Gamma_{i}, i=2,3,4$ and heat flux $\dot{q}$ on surface $\Gamma_{1}$ (a) and the corresponding computational domain (b). The spatially varying thermal conductivity of the body is $k(x, y)$. 
Type 1. Quadratic material gradation: $k=a_{1}\left(1+a_{2} x\right)^{2}\left(1+a_{3} y\right)^{2}$

Type 2. Exponential material gradation: $k=a_{1} e^{\left(1+a_{2} x\right)} e^{\left(1+a_{3} y\right)}$

In this study, the discretization of the physical domain is performed by the elliptic grid generation and the approximation of the derivatives of the field variable (temperature) at grid nodes by algebraic ones is performed by using finite-difference method. In this method, the irregular physical domain (Fig. 1a) is mapped from the $x$ and $y$ physical plane onto the $\xi$ and $\eta$ computational plane (Fig. 1b). Then the heat conduction equation and the boundary conditions (Eqs.(1) to (3)) are transformed from the $x$ and $y$ physical plane to the $\xi$ and $\eta$ computational plane [57]. Here since the thermal conductivity is not constant and is a spatially varying parameter, we can expand Eq. (1) as follows

$$
\frac{\partial k}{\partial x} \frac{\partial T}{\partial x}+k \frac{\partial^{2} T}{\partial x^{2}}+\frac{\partial k}{\partial y} \frac{\partial T}{\partial y}+k \frac{\partial^{2} T}{\partial y^{2}}=0
$$

by simplifying, we get

$$
k\left(\frac{\partial^{2} T}{\partial x^{2}}+\frac{\partial^{2} T}{\partial y^{2}}\right)+\frac{\partial k}{\partial x} \frac{\partial T}{\partial x}+\frac{\partial k}{\partial y} \frac{\partial T}{\partial y}=0
$$

by substituting for $T_{x}, T_{y}, T_{x x}$, and $T_{y y}$ using the transformation relationships and finite-difference expressions [57], Eq. (4) will be

$$
k\left[\frac{1}{J^{2}}\left(\alpha T_{\xi \xi}-2 \beta T_{\xi \eta}+\gamma T_{\eta \eta}\right)\right]+k_{x}\left[\frac{1}{J}\left(y_{\eta} T_{\xi}-y_{\xi} T_{\eta}\right)\right]+k_{y}\left[\frac{1}{J}\left(-x_{\eta} T_{\xi}+x_{\xi} T_{\eta}\right)\right]=0
$$

where for type 1 (quadratic material gradation):

$$
\begin{gathered}
k_{i, j}=a_{1}\left(1+a_{2} x_{i, j}\right)^{2}\left(1+a_{3} y_{i, j}\right)^{2} \\
k_{i, j_{x}}=\frac{\partial k_{i, j}}{\partial x}=2 a_{1} a_{2}\left(1+a_{2} x_{i, j}\right)\left(1+a_{3} y_{i, j}\right)^{2}
\end{gathered}
$$




$$
k_{i, j_{y}}=\frac{\partial k_{i, j}}{\partial y}=2 a_{1} a_{3}\left(1+a_{2} x_{i, j}\right)^{2}\left(1+a_{3} y_{i, j}\right)
$$

and for type 2 (exponential material gradation):

$$
\begin{gathered}
k_{i, j}=a_{1} e^{\left(1+a_{2} x_{i, j}\right)} e^{\left(1+a_{3} y_{i, j}\right)} \\
k_{i, j_{x}}=\frac{\partial k_{i, j}}{\partial x}=a_{2} a_{1} e^{\left(1+a_{2} x_{i, j}\right)} e^{\left(1+a_{3} y_{i, j}\right)} \\
k_{i, j_{y}}=\frac{\partial k_{i, j}}{\partial y}=a_{3} a_{1} e^{\left(1+a_{2} x_{i, j}\right)} e^{\left(1+a_{3} y_{i, j}\right)}
\end{gathered}
$$

and

$$
\begin{gathered}
f_{\xi}=\frac{1}{2}\left(f_{i+1, j}-f_{i-1, j}\right) \\
f_{\eta}=\frac{1}{2}\left(f_{i, j+1}-f_{i, j-1}\right) \\
f_{\xi \xi}=f_{i+1, j}-2 f_{i, j}+f_{i-1, j} \\
f_{\eta \eta}=f_{i, j+1}-2 f_{i, j}+f_{i, j-1} \\
f_{\xi \eta}=\frac{1}{4}\left(f_{i+1, j+1}-f_{i-1, j+1}-f_{i+1, j-1}+f_{i-1, j-1}\right)
\end{gathered}
$$

where $f \equiv x, y, T$. And

$$
\begin{gathered}
\alpha=x_{\eta}^{2}+y_{\eta}^{2} \\
\beta=x_{\xi} x_{\eta}+y_{\xi} y_{\eta} \\
\gamma=x_{\xi}^{2}+y_{\xi}^{2} \\
J=x_{\xi} y_{\eta}-x_{\eta} y_{\xi} \quad \text { (Jacobian of transformation) }
\end{gathered}
$$


are the coefficients obtained from the elliptic grid generation method $[57,58]$. By knowing the values for $a_{l}, l=1,2,3$ as well as $x_{i, j}$ and $y_{i, j}$ (nodal coordinates) from the elliptic grid generation step, the values for $k_{i, j}, k_{i, j_{x}}$, and $k_{i, j_{y}}$ are also known. Eq. (5) may be solved using an algebraic solver such as Maple to obtain an expression for $T_{i, j}$ in the body, as follows

$$
\begin{gathered}
T_{i, j}=\frac{-1}{k_{i, j}(\alpha+\gamma)}\left(0 . 5 \left(-k_{i, j} \alpha T_{i+1, j}-k_{i, j} \alpha T_{i-1, j}+2 k_{i, j} \beta T_{\xi \eta}-k_{i, j} \gamma T_{i, j+1}-k_{i, j} \gamma T_{i, j-1}-k_{i, j} J y_{\eta} T_{\xi}\right.\right. \\
\left.\left.+k_{i, j} J y_{\xi} T_{\eta}+k_{i, j} J x_{\eta} T_{\xi}-k_{i, j_{y}} J x_{\xi} T_{\eta}\right)\right)
\end{gathered}
$$

The term $\frac{\partial T}{\partial n}$ at a boundary surface in the physical domain is related to $\frac{\partial T}{\partial \xi}$ and/or $\frac{\partial T}{\partial \eta}$ at the corresponding transformed boundary surface in the computational domain. At boundary surfaces $\Gamma_{i}, i=1,2,3,4$, we have [57]

$$
\begin{aligned}
& \text { at surface } \Gamma_{1}: \frac{\partial T}{\partial n_{1}}=\frac{-1}{J \sqrt{\gamma}}\left(\gamma T_{\eta}-\beta T_{\xi}\right) \\
& \text { at surface } \Gamma_{2}: \frac{\partial T}{\partial n_{2}}=\frac{1}{J \sqrt{\gamma}}\left(\gamma T_{\eta}-\beta T_{\xi}\right) \\
& \text { at surface } \Gamma_{3}: \frac{\partial T}{\partial n_{3}}=\frac{-1}{J \sqrt{\alpha}}\left(\alpha T_{\xi}-\beta T_{\eta}\right) \\
& \text { at surface } \Gamma_{4}: \frac{\partial T}{\partial n_{4}}=\frac{1}{J \sqrt{\alpha}}\left(\alpha T_{\xi}-\beta T_{\eta}\right)
\end{aligned}
$$

Thus, the boundary condition equation at the surface, say, $\Gamma_{2}$ for the quadratic material gradation type is written as

$$
\left.\dot{q}_{\text {conduction }}\right|_{\Gamma_{2}}=\left.\dot{q}_{\text {convection }}\right|_{\Gamma_{2}}
$$




$$
\begin{gathered}
-k \frac{\partial T}{\partial n_{2}}=h_{2}\left(T_{\Gamma_{2}}-T_{\infty_{2}}\right) \\
-k\left[\frac{1}{J \sqrt{\gamma}}\left(\gamma T_{\eta}-\beta T_{\xi}\right)\right]=h_{2}\left(T_{\Gamma_{2}}-T_{\infty_{2}}\right)
\end{gathered}
$$

at the surface $\Gamma_{2}$, we have

$$
\begin{gathered}
T_{\xi}=\frac{1}{2}\left(T_{i+1, N}-T_{i-1, N}\right) \\
T_{\eta}=\frac{1}{2}\left(3 T_{i, N}-4 T_{i, N-1}+T_{i, N-2}\right)
\end{gathered}
$$

Therefore, Eq. (21) becomes

$$
-k_{i, N}\left[\frac{1}{J \sqrt{\gamma}}\left(\gamma \frac{3 T_{i, N}-4 T_{i, N-1}+T_{i, N-2}}{2}-\beta \frac{T_{i+1, N}-T_{i-1, N}}{2}\right)\right]=h_{2}\left(T_{i, N}-T_{\infty_{2}}\right)
$$

where the coefficients $J, \gamma$, and $\beta$ defined in Eq. (13) are computed using finitedifference coefficients associated with the surface $\Gamma_{2}$. Solving Eq. (24) for $T_{i, N}$ gives the temperature distribution on the boundary surface $\Gamma_{2}$ as follows

$$
T_{i, N}=\frac{k_{i, N}\left(4 \gamma T_{i, N-1}-\gamma T_{i, N-2}+\beta T_{i+1, N}-\beta T_{i-1, N}\right)+2 h_{2} J \sqrt{\gamma} T_{\infty_{2}}}{3 k_{i, N} \gamma+2 h_{2} J \sqrt{\gamma}}
$$

In a similar fashion, we can compute the temperature values on the surfaces $\Gamma_{i}$, $i=1,3,4$.

\section{The inverse analysis}

\subsection{Objective function}

The main objective of the study is to identify the spatially varying thermal conductivity of functionally graded materials. To do so, an inverse analysis is used to estimate the 
thermal conductivity $k=a_{1}\left(1+a_{2} x\right)^{2}\left(1+a_{3} y\right)^{2} \quad$ and $\quad k=a_{1} e^{\left(1+a_{2} x\right)} e^{\left(1+a_{3} y\right)} \quad$ (using the estimation of $a_{1}, a_{2}$, and $a_{3}$ ) separately so that the square of the difference between the computed temperature of the outer surface $\Gamma_{2}$ and the measured temperature of the same surface is minimized. This can be mathematically expressed as

$$
\min \left\{\underset{k(x, y) \text { in } \Omega}{\mathcal{J}}:=C\left\|\mathbf{T}_{\Gamma_{2}}-\mathbf{T}_{m}\right\|^{2}: \text { Eq.(1) in } \Omega \text {, BCs in Eqs.(2)-(3) }\right\}
$$

where $\mathbf{T}_{m}$ is the vector of the measured temperature and $C$ is a positive constant and can be considered as $C=10^{n}, n=0,1,2, \ldots$. The aim of the inverse analysis is to minimize the following objective function expression using optimization of the value of $a_{1}, a_{2}$, and $a_{3}$ :

$$
\mathfrak{J}=C \sum_{i=2}^{M-1}\left(T_{i, N}-T_{(i, N)_{m}}\right)^{2}
$$

\subsection{Sensitivity analysis}

In gradient-based optimization methods, the computation of derivative of the objective function with respect to the unknown variables is required. Thus, in order to compute the sensitivity of the objective function $\mathcal{J}$ defined by Eq. (27) to $a_{l}, l=1,2,3$ we can write

$$
\frac{\partial \mathcal{J}}{\partial a_{l}}=2 C \sum_{i=2}^{M-1}\left(T_{i, N}-T_{(i, N)_{m}}\right) \frac{\partial T_{i, N}}{\partial a_{l}}=2 \sum_{i=2}^{M-1}\left(T_{i, N}-T_{(i, N)_{m}}\right) C \frac{\partial T_{i, N}}{\partial a_{l}}
$$

where $l=1,2,3$. In Eq. (28), $C \frac{\partial T_{i, N}}{\partial a_{l}}(l=1,2,3)$ are called the sensitivity coefficients and may be explicitly expressed by taking derivative of Eq. (25) with respect to the variable thermal conductivity components $a_{l}, l=1,2,3$. For the case $k=a_{1}\left(1+a_{2} x\right)^{2}\left(1+a_{3} y\right)^{2}$ we can write Eq. (25) as 


$$
T_{i, N}=\frac{a_{1}\left(1+a_{2} x_{i, N}\right)^{2}\left(1+a_{3} y_{i, N}\right)^{2}\left(4 \gamma T_{i, N-1}-\gamma T_{i, N-2}+\beta T_{i+1, N}-\beta T_{i-1, N}\right)+2 h_{2} J \sqrt{\gamma} T_{\infty_{2}}}{3 a_{1}\left(1+a_{2} x_{i, N}\right)^{2}\left(1+a_{3} y_{i, N}\right)^{2} \gamma+2 h_{2} J \sqrt{\gamma}}
$$

Now the sensitivity coefficients $C \frac{\partial T_{i, N}}{\partial a_{l}}(l=1,2,3)$ can be explicitly expressed by differentiating the obtained expression for $T_{i, N}$ with respect to $a_{l}, l=1,2,3$, as follows

$$
\begin{aligned}
& \frac{\partial T_{i, N}}{\partial a_{1}}=2 h_{2} J \sqrt{\gamma}\left(-4 a_{2} x_{i, N} a_{3} y_{i, N} \gamma T_{i, N-2}+8 a_{2} x_{i, N} a_{3} y_{i, N} \beta T_{\xi}+8 a_{2} x_{i, N} a_{3}^{2} y_{i, N}^{2} \gamma T_{i, N-1}\right. \\
& -2 a_{2} x_{i, N} a_{3}^{2} y_{i, N}^{2} \gamma T_{i, N-2}+4 a_{2} x_{i, N} a_{3}^{2} y_{i, N}^{2} \beta T_{\xi}+8 a_{2}^{2} x_{i, N}^{2} a_{3} y_{i, N} \gamma T_{i, N-1}-2 a_{2}^{2} x_{i, N}^{2} a_{3} y_{i, N} \gamma T_{i, N-2} \\
& +2 \beta T_{\xi}+16 a_{2} x_{i, N} a_{3} y_{i, N} \gamma T_{i, N-1}-3 \gamma T_{\infty_{2}}+8 a_{3} y_{i, N} \gamma T_{i, N-1}-2 a_{3} y_{i, N} \gamma T_{i, N-2}+4 a_{3} y_{i, N} \beta T_{\xi} \\
& +4 a_{3}^{2} y_{i, N}^{2} \gamma T_{i, N-1}-a_{3}^{2} y_{i, N}^{2} \gamma T_{i, N-2}+2 a_{3}^{2} y_{i, N}^{2} \beta T_{\xi}+8 a_{2} x_{i, N} \gamma T_{i, N-1}-2 a_{2} x_{i, N} \gamma T_{i, N-2}+4 a_{2} x_{i, N} \beta T_{\xi} \\
& +4 a_{2}^{2} x_{i, N}^{2} \gamma T_{i, N-1}-a_{2}^{2} x_{i, N}^{2} \gamma T_{i, N-2}+2 a_{2}^{2} x_{i, N}^{2} \beta T_{\xi}+4 \gamma T_{i, N-1}-\gamma T_{i, N-2}+4 a_{2}^{2} x_{i, N}^{2} a_{3} y_{i, N} \beta T_{\xi} \\
& +4 a_{2}^{2} x_{i, N}^{2} a_{3}^{2} y_{i, N}^{2} \gamma T_{i, N-1}-a_{2}^{2} x_{i, N}^{2} a_{3}^{2} y_{i, N}^{2} \gamma T_{i, N-2}+2 a_{2}^{2} x_{i, N}^{2} a_{3}^{2} y_{i, N}^{2} \beta T_{\xi}-6 \gamma T_{\infty_{2}} a_{3} y_{i, N}-3 \gamma T_{\infty_{2}} a_{3}^{2} y_{i, N}^{2} \\
& -6 \gamma T_{\infty_{2}} a_{2} x_{i, N}-3 \gamma T_{\infty_{2}} a_{2}^{2} x_{i, N}^{2}-12 \gamma T_{\infty_{2}} a_{2} x_{i, N} a_{3} y_{i, N}-6 \gamma T_{\infty_{2}} a_{2} x_{i, N} a_{3}^{2} y_{i, N}^{2}-6 \gamma T_{\infty_{2}} a_{2}^{2} x_{i, N}^{2} a_{3} y_{i, N} \\
& \left.-3 \gamma T_{\infty_{2}} a_{2}^{2} x_{i, N}^{2} a_{3}^{2} y_{i, N}^{2}\right) /\left(12 a_{1} a_{2} x_{i, N} a_{3} y_{i, N} \gamma+6 a_{1} a_{2} x_{i, N} a_{3}^{2} y_{i, N}^{2} \gamma+6 a_{1} a_{2}^{2} x_{i, N}^{2} a_{3} y_{i, N} \gamma+3 a_{1} a_{2}^{2} x_{i, N}^{2} a_{3}^{2} y_{i, N}^{2} \gamma\right. \\
& \left.+6 a_{1} a_{3} y_{i, N} \gamma+3 a_{1} a_{3}^{2} y_{i, N}^{2} \gamma+6 a_{1} a_{2} x_{i, N} \gamma+3 a_{1} a_{2}^{2} x_{i, N}^{2} \gamma+3 a_{1} \gamma+2 h_{2} J \sqrt{\gamma}\right)^{2} \\
& \frac{\partial T_{i, N}}{\partial a_{2}}=4 a_{1} x_{i, N} h_{2} J \sqrt{\gamma}\left(2 \beta T_{\xi}+4 \gamma T_{i, N-1}-\gamma T_{i, N-2}-3 \gamma T_{\infty_{2}}+8 a_{3} y_{i, N} \gamma T_{i, N-1}-2 a_{3} y_{i, N} \gamma T_{i, N-2}\right. \\
& +4 a_{3} y_{i, N} \beta T_{\xi}+4 a_{3}^{2} y_{i, N}^{2} \gamma T_{i, N-1}-a_{3}^{2} y_{i, N}^{2} \gamma T_{i, N-2}+2 a_{3}^{2} y_{i, N}^{2} \beta T_{\xi}+4 a_{2} x_{i, N} \gamma T_{i, N-1}-a_{2} x_{i, N} \gamma T_{i, N-2} \\
& +2 a_{2} x_{i, N} \beta T_{\xi}-2 a_{2} x_{i, N} a_{3} y_{i, N} \gamma T_{i, N-2}+4 a_{2} x_{i, N} a_{3} y_{i, N} \beta T_{\xi}+4 a_{2} x_{i, N} a_{3}^{2} y_{i, N}^{2} \gamma T_{i, N-1}-a_{2} x_{i, N} a_{3}^{2} y_{i, N}^{2} \gamma T_{i, N-2} \\
& +2 a_{2} x_{i, N} a_{3}^{2} y_{i, N}^{2} \beta T_{\xi}+8 a_{2} x_{i, N} a_{3} y_{i, N} \gamma T_{i, N-1}-6 \gamma T_{\infty_{2}} a_{3} y_{i, N}-3 \gamma T_{\infty_{2}} a_{3}^{2} y_{i, N}^{2}-3 \gamma T_{\infty_{2}} a_{2} x_{i, N} \\
& \left.-6 \gamma T_{\infty_{2}} a_{2} x_{i, N} a_{3} y_{i, N}-3 \gamma T_{\infty_{2}} a_{2} x_{i, N} a_{3}^{2} y_{i, N}^{2}\right) /\left(12 a_{1} a_{2} x_{i, N} a_{3} y_{i, N} \gamma+6 a_{1} a_{2} x_{i, N} a_{3}^{2} y_{i, N}^{2} \gamma+6 a_{1} a_{2}^{2} x_{i, N}^{2} a_{3} y_{i, N} \gamma\right. \\
& \left.+3 a_{1} a_{2}^{2} x_{i, N}^{2} a_{3}^{2} y_{i, N}^{2} \gamma+6 a_{1} a_{3} y_{i, N} \gamma+3 a_{1} a_{3}^{2} y_{i, N}^{2} \gamma+6 a_{1} a_{2} x_{i, N} \gamma+3 a_{1} a_{2}^{2} x_{i, N}^{2} \gamma+3 a_{1} \gamma+2 h_{2} J \sqrt{\gamma}\right)^{2}
\end{aligned}
$$




$$
\begin{aligned}
& \frac{\partial T_{i, N}}{\partial a_{3}}=4 a_{1} y_{i, N} h_{2} J \sqrt{\gamma}\left(2 \beta T_{\xi}+4 \gamma T_{i, N-1}-\gamma T_{i, N-2}-3 \gamma T_{\infty_{2}}+4 a_{3} y_{i, N} \gamma T_{i, N-1}-a_{3} y_{i, N} \gamma T_{i, N-2}\right. \\
& +2 a_{3} y_{i, N} \beta T_{\xi}+8 a_{2} x_{i, N} \gamma T_{i, N-1}-2 a_{2} x_{i, N} \gamma T_{i, N-2}+4 a_{2} x_{i, N} \beta T_{\xi}+4 a_{2}^{2} x_{i, N}^{2} \gamma T_{i, N-1}-a_{2}^{2} x_{i, N}^{2} \gamma T_{i, N-2} \\
& +2 a_{2}^{2} x_{i, N}^{2} \beta T_{\xi}-2 a_{2} x_{i, N} a_{3} y_{i, N} \gamma T_{i, N-2}+4 a_{2} x_{i, N} a_{3} y_{i, N} \beta T_{\xi}+4 a_{2}^{2} x_{i, N}^{2} a_{3} y_{i, N} \gamma T_{i, N-1}-a_{2}^{2} x_{i, N}^{2} a_{3} y_{i, N} \gamma T_{i, N-2} \\
& +8 a_{2} x_{i, N} a_{3} y_{i, N} \gamma T_{i, N-1}+2 a_{2}^{2} x_{i, N}^{2} a_{3} y_{i, N} \beta T_{\xi}-3 \gamma T_{\infty_{2}} a_{3} y_{i, N}-6 \gamma T_{\infty_{2}} a_{2} x_{i, N}-3 \gamma T_{\infty_{2}} a_{2}^{2} x_{i, N}^{2} \\
& \left.-6 \gamma T_{\infty_{2}} a_{2} x_{i, N} a_{3} y_{i, N}-3 \gamma T_{\infty_{2}} a_{2}^{2} x_{i, N}^{2} a_{3} y_{i, N}\right) /\left(12 a_{1} a_{2} x_{i, N} a_{3} y_{i, N} \gamma+6 a_{1} a_{2} x_{i, N} a_{3}^{2} y_{i, N}^{2} \gamma+6 a_{1} a_{2}^{2} x_{i, N}^{2} a_{3} y_{i, N} \gamma\right. \\
& \left.+3 a_{1} a_{2}^{2} x_{i, N}^{2} a_{3}^{2} y_{i, N}^{2} \gamma+6 a_{1} a_{3} y_{i, N} \gamma+3 a_{1} a_{3}^{2} y_{i, N}^{2} \gamma+6 a_{1} a_{2} x_{i, N} \gamma+3 a_{1} a_{2}^{2} x_{i, N}^{2} \gamma+3 a_{1} \gamma+2 h_{2} J \sqrt{\gamma}\right)^{2} \text { (32) }
\end{aligned}
$$

For the case $k=a_{1} e^{\left(1+a_{2} x\right)} e^{\left(1+a_{3} y\right)}$ we can write Eq. (25) as

$$
T_{i, N}=\frac{a_{1} e^{\left(1+a_{2} x_{i, N}\right)} e^{\left(1+a_{3} y_{i, N}\right)}\left(4 \gamma T_{i, N-1}-\gamma T_{i, N-2}+\beta T_{i+1, N}-\beta T_{i-1, N}\right)+2 h_{2} J \sqrt{\gamma} T_{\infty_{2}}}{3 a_{1} e^{\left(1+a_{2} x_{i, N}\right)} e^{\left(1+a_{3} y_{i, N}\right)} \gamma+2 h_{2} J \sqrt{\gamma}}
$$

and the sensitivity coefficients $C \frac{\partial T_{i, N}}{\partial a_{l}}(l=1,2,3)$ can be written as

$$
\begin{gathered}
\frac{\partial T_{i, N}}{\partial a_{1}}=\frac{2 e^{\left(2+a_{2} x_{i, N}+a_{3} y_{i, N}\right)} h_{2} J \sqrt{\gamma}\left(4 \gamma T_{i, N-1}-\gamma T_{i, N-2}+\beta T_{i+1, N}-\beta T_{i-1, N}-3 \gamma T_{\infty_{2}}\right)}{\left(3 a_{1} e^{\left(2+a_{2} x_{i, N}+a_{3} y_{i, N}\right)} \gamma+2 h_{2} J \sqrt{\gamma}\right)^{2}} \\
\frac{\partial T_{i, N}}{\partial a_{2}}=\frac{2 a_{1} x_{i, N} e^{\left(2+a_{2} x_{i, N}+a_{3} y_{i, N}\right)} h_{2} J \sqrt{\gamma}\left(4 \gamma T_{i, N-1}-\gamma T_{i, N-2}+\beta T_{i+1, N}-\beta T_{i-1, N}-3 \gamma T_{\infty_{2}}\right)}{\left(3 a_{1} e^{\left(2+a_{2} x_{i, N}+a_{3} y_{i, N}\right)} \gamma+2 h_{2} J \sqrt{\gamma}\right)^{2}} \\
\frac{\partial T_{i, N}}{\partial a_{3}}=\frac{2 a_{1} y_{i, N} e^{\left(2+a_{2} x_{i, N}+a_{3} y_{i, N}\right)} h_{2} J \sqrt{\gamma}\left(4 \gamma T_{i, N-1}-\gamma T_{i, N-2}+\beta T_{i+1, N}-\beta T_{i-1, N}-3 \gamma T_{\infty_{2}}\right)}{\left(3 a_{1} e^{\left(2+a_{2} x_{i, N}+a_{3} y_{i, N}\right)} \gamma+2 h_{2} J \sqrt{\gamma}\right)^{2}}
\end{gathered}
$$

Therefore, the sensitivity coefficients $C \frac{\partial T_{i, N}}{\partial a_{l}}$ can be computed in only one single direct problem solution without the need for solving the adjoint equation. The sensitivity matrix Ja can be explicitly written as 


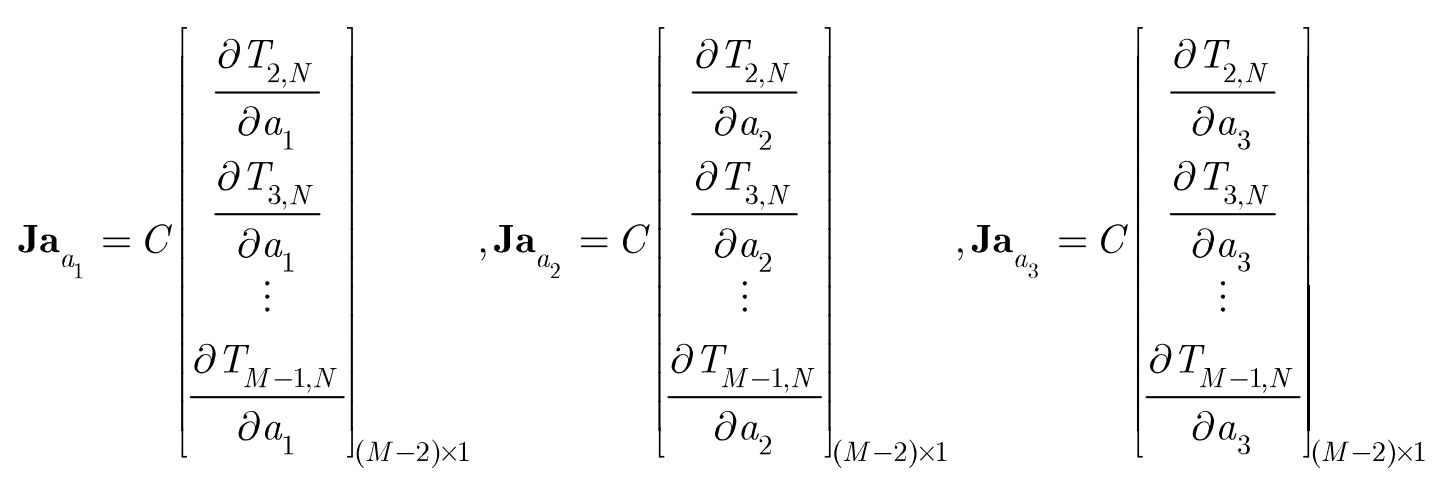

\subsection{The Conjugate Gradient Method (CGM)}

In this study, the inverse heat transfer problem is solved using the conjugate gradient optimization method. The objective function given by Eq. (27) is minimized by searching along the direction of descent $d^{(\mathrm{k})}$ using a search step length $\beta^{(\mathrm{k})}$.

$$
f^{(\mathrm{k}+1)}=f^{(\mathrm{k})}-\beta^{(\mathrm{k})} d^{(\mathrm{k})}
$$

where $f \equiv a_{1}, a_{2}, a_{3}$. The direction of descent of the current iteration is obtained as a linear combination of the direction of descent of the previous iteration and the gradient direction $\nabla \mathcal{J}^{(\mathrm{k})}$. Therefore,

$$
d^{(\mathrm{k})}=\nabla \mathfrak{J}^{(\mathrm{k})}+\gamma^{(\mathrm{k})} d^{(\mathrm{k}-1)}
$$

The Polak-Ribiere formula [59] is employed to calculate the conjugation coefficient:

$$
\gamma^{(\mathrm{k})}=\frac{\left[\nabla \mathfrak{J}^{(\mathrm{k})}\right]^{\mathrm{T}}\left(\nabla \mathfrak{J}^{(\mathrm{k})}-\nabla \mathfrak{J}^{(\mathrm{k}-1)}\right)}{\left\|\nabla \mathfrak{J}^{(\mathrm{k}-1)}\right\|^{2}}=\frac{\left[\nabla \mathfrak{J}^{(\mathrm{k})}\right]^{\mathrm{T}}\left(\nabla \mathfrak{J}^{(\mathrm{k})}-\nabla \mathfrak{J}^{(\mathrm{k}-1)}\right)}{\left[\nabla \mathfrak{J}^{(\mathrm{k}-1)}\right]^{\mathrm{T}} \nabla \mathfrak{J}^{(\mathrm{k}-1)}}
$$

The search step length is given as follows [50]

$$
\beta^{(\mathrm{k})}=\frac{\left[\mathbf{J a}^{(\mathrm{k})} d^{(\mathrm{k})}\right]^{\mathrm{T}}\left[\mathbf{T}_{i, N}-\mathbf{T}_{(i, N)_{m}}\right]}{\left[\mathbf{J a}^{(\mathrm{k})} d^{(\mathrm{k})}\right]^{\mathrm{T}}\left[\mathbf{J} \mathbf{a}^{(\mathrm{k})} d^{(\mathrm{k})}\right]}
$$




\subsubsection{Optimization algorithm}

The following algorithm represents the direct and inverse analysis steps used to estimate the spatially varying thermal conductivity of functionally graded materials in steadystate heat conduction problems:

1. Specify the physical domain, the boundary conditions, type of material gradation, and the measured outer surface temperature.

2. Generate the boundary-fitted grid using the elliptic grid generation method.

3. Solve the direct problem of finding the temperature values at any grid points of the physical domain using an initial variable thermal conductivity (initial guess for $a_{1}, a_{2}, a_{3}$ ) .

4. Using Eq. (27), compute the objective function $\left(\mathfrak{J}^{(\mathrm{k})}\right)$.

5. If value of the objective function obtained in step 4 is less than the specified stopping criterion, the optimization is finished. Otherwise, go to step 6 .

6. Compute the sensitivity matrix $\mathbf{J a}_{a_{1}}$ from Eq. (37)

7. Compute the gradient direction $\nabla \mathfrak{J}_{a_{1}}^{(\mathrm{k})}$ from Eq. (28).

8. Compute the conjugation coefficient $\gamma_{a_{1}}^{(\mathrm{k})}$ from Eq. (40). For $\mathrm{k}=0$, set $\gamma^{(0)}=0$.

9. Compute the directions of descent $d_{a_{1}}^{(\mathrm{k})}$ from Eq. (39).

10. Compute the search step-length $\beta_{a_{1}}^{(\mathrm{k})}$ from Eq. (41).

11. From Eq. (38), evaluate the new value for $a_{1}$.

12. With new value for $a_{1}$, repeat the steps 6 to 11 for $a_{2}$.

13. With new values for $a_{1}$ and $a_{2}$, repeat the steps 6 to 11 for $a_{3}$. 
14. Set the next iteration $(\mathrm{k}=\mathrm{k}+1)$ and return to the step 2 .

\section{4 stopping criterion}

Without measurement errors, the following criterion is used to terminate the minimization process

$$
\mathfrak{J}^{(\mathrm{k})}<\varepsilon
$$

where $\varepsilon$ is a small specified number. In this study, for the case of no measurement error, $\varepsilon=10^{-4}$ and $\varepsilon=10^{-5}$ (depending on the value of $C$ ). However, the measured temperatures contain errors and the objective function value will not be zero at the end of the iterative process. In this case, the Discrepancy Principle is used to stop the iterative procedure. In the Discrepancy Principle, the solution is assumed to be sufficiently accurate when the difference between computed and measured temperatures is of the order of magnitude of the measurement errors, that is,

$$
\left|\mathbf{T}_{\text {computed }}-\mathbf{T}_{\text {measured }}\right| \approx \sigma
$$

where $\sigma$ is the standard deviation of the measurement errors. In the present analysis, $\sigma$ is assumed constant. By substituting Eq. (43) into Eq. (27) (objective function definition) we can obtain the following value for $\varepsilon$

$$
\varepsilon=C(M-2) \sigma^{2}
$$

Then the iterative procedure is stopped when the following criterion is satisfied [50]

$$
\mathfrak{J}^{(\mathrm{k})}<\varepsilon
$$

\subsection{Identifiability condition}

As given in the optimization algorithm, the parameters $a_{1}, a_{2}$, and $a_{3}$ are estimated separately and successively at each iteration. They can be estimated as long as the identifiability condition is met. By considering Eq. (38), $f^{(\mathrm{k}+1)}=f^{(\mathrm{k})}-\beta^{(\mathrm{k})} d^{(\mathrm{k})}$, where 
$f \equiv a_{1}, a_{2}, a_{3}$, it can be seen that if $\beta^{(\mathrm{k})} d^{(\mathrm{k})}=0$ where $d^{(\mathrm{k})}=\nabla \mathcal{J}^{(\mathrm{k})}+\gamma^{(\mathrm{k})} d^{(\mathrm{k}-1)}$ then the parameters $a_{1}, a_{2}$, and $a_{3}$ cannot be updated. From the expression for the gradient of the objective function with respect to the parameters $a_{1}, a_{2}$, and $a_{3}$, Eq. (28),

$$
\frac{\partial \mathcal{J}}{\partial a_{l}}=2\left[\mathbf{J a}_{a_{l}}\right]^{\mathrm{T}}\left[\mathbf{T}_{i, N}-\mathbf{T}_{(i, N)_{m}}\right], \quad l=1,2,3
$$

as well as from the expression for the direction of descent, Eq. (39), it can be concluded that if $\mathbf{J a}_{a_{l}}=0$, then $\nabla \mathfrak{J}^{(\mathrm{k})}=0$ and hence $d^{(\mathrm{k})}=0$. Thus we can write

$$
\left[\mathbf{J a}^{(\mathrm{k})}\right]_{(M-2) \times 1} \neq 0
$$

which is the so-called identifiability condition for the estimation of the parameters $a_{1}$, $a_{2}$, and $a_{3}$. A zero matrix is a matrix in which all entries are zero, that is, if $\mathbf{J a}=0$ then

$$
J a_{1,1}=J a_{2,1}=J a_{3,1}=\ldots=J a_{M-2,1}=0
$$

By considering the sensitivity coefficients given by Eqs. (30)-(32) for the quadratic gradation type and Eqs. (34)-(36) for the exponential gradation type, we can see that the only possibility to meet the conditions given in Eq. (48) is

$$
h_{2}=0 \quad \text { or } \quad a_{1}=0
$$

Therefore the identifiability conditions for the parameter estimation problems given in this study are as follows

$$
h_{2} \neq 0 \quad \text { and } \quad a_{1} \neq 0
$$

from the definitions given for the thermal conductivity gradations, $k=a_{1}\left(1+a_{2} x\right)^{2}\left(1+a_{3} y\right)^{2}$ and $k=a_{1} e^{\left(1+a_{2} x\right)} e^{\left(1+a_{3} y\right)}$, it is evident that if $a_{1}=0$ then $k=0$. If $h_{2}=0$ and $a_{1}=0$, then the denominator of the expressions for the Jacobian matrices entries become zero. 
Moreover, it can be noted that the denominator of the expression for $\beta^{(\mathrm{k})}$ should not be zero. Thus if

$$
\left[\mathbf{J a}^{(\mathrm{k})}\right]_{1 \times(M-2)}^{\mathrm{T}}\left[\mathbf{J a}^{(\mathrm{k})}\right]_{(M-2) \times 1}=0
$$

by expanding Eq. (51) we obtain

$$
J a_{1,1}^{2}+J a_{2,1}^{2}+J a_{3,1}^{2}+\ldots+J a_{M-2,1}^{2}=0
$$

and the only possibility is that

$$
J a_{1,1}=J a_{2,1}=J a_{3,1}=\ldots=J a_{M-2,1}=0
$$

which again gives rise to the previous conditions of

$$
h_{2}=0 \quad \text { or } \quad a_{1}=0
$$

Therefore, the parameters $a_{1}, a_{2}$, and $a_{3}$ cannot be simultaneously estimated using the iterative procedure if $h_{2}=0$ or $a_{1}=0$. Moreover, it can be seen that if $x_{i, N}=0$ ( $i=2, \ldots, M-1)$ and $y_{i, N}=0 \quad(i=2, \ldots, M-1)$, then $\frac{\partial T_{i, N}}{\partial a_{2}}=0$ and $\frac{\partial T_{i, N}}{\partial a_{3}}=0$ respectively, and these parameters cannot be estimated.

\subsection{Linearly independent sensitivity coefficients}

In inverse problems, it is desirable to have linearly-independent sensitivity coefficients with large magnitude. The sensitivity vectors $\mathbf{J a}_{a_{1}}, \mathbf{J a}_{a_{2}}$, and $\mathbf{J a}_{a_{3}}$ are linearly independent if the equation

$$
c_{1} \mathbf{J a}_{a_{1}}+c_{2} \mathbf{J a}_{a_{2}}+c_{3} \mathbf{J a}_{a_{3}}=0
$$

has no solution other than

$$
c_{1}=c_{2}=c_{3}=0
$$

where $c_{1}, c_{2}$, and $c_{3}$ are scalars. Eq. (55) can be written as 


$$
c_{1}\left[\begin{array}{c}
J a_{a_{1_{(1,1)}}} \\
J a_{a_{1_{(2,1)}}} \\
\vdots \\
J a_{a_{(i, 1)}}
\end{array}\right]+c_{2}\left[\begin{array}{c}
J a_{a_{2_{(1,1)}}} \\
J a_{a_{2_{(2,1)}}} \\
\vdots \\
J a_{a_{2_{(i, 1)}}}
\end{array}\right]+c_{3}\left[\begin{array}{c}
J a_{a_{3(1,1)}} \\
J a_{a_{3(2,1)}} \\
\vdots \\
J a_{a_{3(i, 1)}}
\end{array}\right]=0
$$

or

$$
\begin{aligned}
& c_{1} J a_{a_{1_{(1,1)}}}+c_{2} J a_{a_{2_{(1,1)}}}+c_{3} J a_{a_{3(1,1)}}=0 \\
& c_{1} J a_{a_{1_{(2,1)}}}+c_{2} J a_{a_{2_{(2,1)}}}+c_{3} J a_{a_{3(2,1)}}=0 \\
& c_{1} J a_{a_{1_{(i, 1)}}}+c_{2} J a_{a_{2_{(i, 1)}}}+c_{3} J a_{a_{3_{(i, 1)}}}=0
\end{aligned}
$$

Eq. (58) can be written as

$$
\left[\begin{array}{ccc}
J a_{a_{1_{(1,1)}}} & J a_{a_{2_{(1,1)}}} & J a_{a_{3_{(1,1)}}} \\
J a_{a_{1_{(2,1)}}} & J a_{a_{2_{(2,1)}}} & J a_{a_{3(2,1)}} \\
\vdots & \vdots & \vdots \\
J a_{a_{1_{(i, 1)}}} & J a_{a_{2_{(i, 1)}}} & J a_{a_{3(i, 1)}}
\end{array}\right]\left\{\begin{array}{l}
c_{1} \\
c_{2} \\
c_{3}
\end{array}\right\}=\left\{\begin{array}{l}
0 \\
0 \\
0
\end{array}\right\}
$$

If $i=1$ (only one sensor is used to measure the temperature), then Eq. (58) (or Eq. (59) ) becomes

$$
c_{1} J a_{a_{1(1,1)}}+c_{2} J a_{a_{2_{(1,1)}}}+c_{3} J a_{a_{3(1,1)}}=0
$$

which has a solution given by

$$
c_{1}=c_{1}, c_{2}=-\frac{c_{1} J a_{a_{1_{(1,1)}}}+c_{3} J a_{a_{3(1,1)}}}{J a_{a_{2(1,1)}}}, c_{3}=c_{3}
$$

where $c_{1}$ and $c_{3}$ can be chosen arbitrarily and $c_{2}$ is dependent on $c_{1}$ and $c_{3}$. Therefore, the sensitivity coefficients $J a_{a_{1_{(1,1)}}}, J a_{a_{1_{(1,1)}}}$, and $J a_{a_{1_{(1,1)}}}$ are linearly dependent. 
If $i=2$ (two sensors are used to measure the temperature), then Eq. (59) becomes

$$
\left[\begin{array}{l}
c_{1} J a_{a_{1_{(1,1)}}}+c_{2} J a_{a_{2_{(1,1)}}}+c_{3} J a_{a_{3_{(1,1)}}}=0 \\
c_{1} J a_{a_{1_{(2,1)}}}+c_{2} J a_{a_{2_{(2,1)}}}+c_{3} J a_{a_{3(2,1)}}=0
\end{array}\right]
$$

which has a solution given by

$c_{1}=c_{1}, c_{2}=\frac{c_{1}\left(-J a_{a_{1_{(1,1)}}} J a_{a_{3_{(2,1)}}}+J a_{a_{3_{(1,1)}}} J a_{a_{1_{(2,1)}}}\right)}{J a_{a_{2_{(1,1)}}} J a_{a_{3_{(2,1)}}}-J a_{a_{2_{(2,1)}}} J a_{a_{a_{(1,1)}}}}, c_{3}=-\frac{c_{1}\left(J a_{a_{2_{(1,1)}}} J a_{a_{1_{(2,1)}}}-J a_{a_{2_{(2,1)}}} J a_{a_{a_{(1,1)}}}\right)}{J a_{a_{2_{(1,1)}}} J a_{a_{3(2,1)}}-J a_{a_{a_{(2,1)}}} J a_{a_{3(1,1)}}}$

where $c_{1}$ can be chosen arbitrarily and $c_{2}$ and $c_{3}$ are dependent on $c_{1}$. Therefore, the sensitivity vectors $\mathbf{J a}_{a_{1}}, \mathbf{J} \mathbf{a}_{a_{2}}$, and $\mathbf{J} \mathbf{a}_{a_{3}}$ are linearly dependent.

If $i>2$ (more than two sensors are used to measure the temperature), then the solution for Eq. (59), for the sensitivity coefficients obtained in this study, becomes

$$
c_{1}=c_{2}=c_{3}=0
$$

It means that when more than two sensors are used to measure the temperature, the sensitivity vectors given by Eq. (37) become linearly independent. In this study, $(M-2)$ sensors, as simulated measurements, ( $M$ is the number of nodes on the boundary surface $\Gamma_{2}$, as shown in Fig. 1b) are used which is much larger than two sensors.

\subsection{Sensor (simulated measurement) place inside the heat-conducting body}

It can be shown that the spatially varying thermal conductivity (quadratic and exponential material gradation forms) considered in this study cannot be estimated if the sensor is placed inside the body. From Eq. (5) or Eq. (14) it can be seen that the variable $a_{1}$ (assuming $a_{1} \neq 0$ ) is common to all terms and hence can be factored out. 
Thus if the sensor is placed at the node $(i, j)(i=2, \ldots, M-1 ; j=2, \ldots, N-1)$ inside the body to measure the temperature, then we obtain

$$
\frac{\partial T_{i, j}}{\partial a_{1}}=0
$$

which means that the estimation of the variable $a_{1}$ using the temperature data from a sensor inside the body is not feasible. For the variables $a_{2}$ and $a_{3}$ we obtain

For the quadratic material gradation:

$$
\begin{gathered}
\frac{\partial T_{i, j}}{\partial a_{2}}=-\frac{J\left(y_{\xi} T_{\eta}-y_{\eta} T_{\xi}\right)}{(\alpha+\gamma)\left(1+a_{2} x_{i, j}\right)^{2}} \\
\frac{\partial T_{i, j}}{\partial a_{3}}=\frac{J\left(x_{\xi} T_{\eta}-x_{\eta} T_{\xi}\right)}{(\alpha+\gamma)\left(1+a_{3} y_{i, j}\right)^{2}}
\end{gathered}
$$

For the exponential material gradation:

$$
\begin{gathered}
\frac{\partial T_{i, j}}{\partial a_{2}}=-\frac{0.5 J\left(y_{\xi} T_{\eta}-y_{\eta} T_{\xi}\right)}{(\alpha+\gamma)} \\
\frac{\partial T_{i, j}}{\partial a_{3}}=\frac{0.5 J\left(x_{\xi} T_{\eta}-x_{\eta} T_{\xi}\right)}{(\alpha+\gamma)}
\end{gathered}
$$

where all variables in the computational domain are calculated at the node $(i, j)$ inside the body.

However, for other forms of spatial variation of the thermal conductivity such as $k_{i, j}=a_{1}+a_{2} x_{i, j}+a_{3} y_{i, j}$ or $k_{i, j}=a_{1}+a_{2} x_{i, j}+a_{3} y_{i, j}+a_{4} x_{i, j} y_{i, j}$ (which are not considered in this study) we can place the measurement sensor inside the body to estimate the variables $a_{1}, a_{2}, a_{3}$, and $a_{4}$. For example, for the form $k_{i, j}=a_{1}+a_{2} x_{i, j}+a_{3} y_{i, j}$, we have 


$$
\begin{gathered}
\frac{\partial T_{i, j}}{\partial a_{1}}=\frac{0.5 J\left(-a_{2} y_{\eta} T_{\xi}+a_{2} y_{\xi} T_{\eta}+a_{3} x_{\eta} T_{\xi}-a_{3} x_{\xi} T_{\eta}\right)}{\left(a_{1}+a_{2} x_{i, j}+a_{3} y_{i, j}\right)^{2}(\alpha+\gamma)} \\
\frac{\partial T_{i, j}}{\partial a_{2}}=-\frac{0.5 J\left(-y_{\eta} T_{\xi} a_{3} y_{i, j}+y_{\xi} T_{\eta} a_{3} y_{i, j}-x_{i, j} a_{3} x_{\eta} T_{\xi}+x_{i, j} a_{3} x_{\xi} T_{\eta}-y_{\eta} T_{\xi} a_{1}+y_{\xi} T_{\eta} a_{1}\right)}{\left(a_{1}+a_{2} x_{i, j}+a_{3} y_{i, j}\right)^{2}(\alpha+\gamma)} \\
\frac{\partial T_{i, j}}{\partial a_{3}}=\frac{0.5 J\left(-x_{\eta} T_{\xi} a_{1}+x_{\xi} T_{\eta} a_{1}-x_{\eta} T_{\xi} a_{2} x_{i, j}+x_{\xi} T_{\eta} a_{2} x_{i, j}-y_{i, j} a_{2} y_{\eta} T_{\xi}+y_{i, j} a_{2} y_{\xi} T_{\eta}\right)}{\left(a_{1}+a_{2} x_{i, j}+a_{3} y_{i, j}\right)^{2}(\alpha+\gamma)}
\end{gathered}
$$

\subsection{Statistical analysis for parameter estimation}

After the minimization of the ordinary least-squares norm given in Eq. (27) and the estimation of the unknown parameters $a_{1}, a_{2}$, and $a_{3}$, the accuracy of the estimated values for the parameters can be evaluated using a statistical analysis. If the measurement errors are additive, unbiased (having zero mean), uncorrelated and have constant variance, then confidence intervals at the $99 \%$ confidence level for the estimated parameters are obtained as $[50,60]$

$$
a_{i}-2.576 \sigma_{a_{i}} \leq a_{i} \leq a_{i}+2.576 \sigma_{a_{i}}, \quad i=1,2,3
$$

where $\sigma_{a_{1}}, \sigma_{a_{2}}$, and $\sigma_{a_{3}}$ are the standard deviations for the estimated parameters $a_{1}$, $a_{2}$, and $a_{3}$, respectively, and are obtained from the covariance matrix, $V$, of the estimated parameters as follows

$$
\sigma_{a_{i}}=\sqrt{V_{a_{i}}}
$$

The covariance matrix, $V$, is given by $[32,50]$

$$
V=\sigma^{2}\left(\mathbf{J a}^{\mathrm{T}} \mathbf{J} \mathbf{a}\right)^{-1}
$$

where $\sigma$ is the standard deviation of the measurement errors and is assumed to be constant (in this study, $\sigma=0.5$ ). For the sensitivity vectors $\mathbf{J a}_{a_{1}}, \mathbf{J} \mathbf{a}_{a_{2}}$, and $\mathbf{J a}_{a_{3}}$, the covariance matrices are scalars and are given by 


$$
\begin{gathered}
V=\sigma^{2}\left(\mathbf{J a}^{\mathrm{T}} \mathbf{J a}\right)^{-1}=\sigma^{2}\left(J a_{1,1}^{2}+J a_{2,1}^{2}+J a_{3,1}^{2}+\ldots+J a_{M-2,1}^{2}\right)^{-1} \\
=\frac{\sigma^{2}}{J a_{1,1}^{2}+J a_{2,1}^{2}+J a_{3,1}^{2}+\ldots+J a_{M-2,1}^{2}}
\end{gathered}
$$

Thus from Eq. (74), the standard deviations for the estimated parameters can be obtained as

$$
\sigma_{a_{i}}=\sqrt{V_{a_{i}}}=\frac{\sigma}{\sqrt{J a_{a_{i_{1,1}}}^{2}+J a_{a_{i_{2,1}}}^{2}+J a_{a_{i_{3,1}}}^{2}+\ldots+J a_{a_{i_{M-2,1}}}^{2}}}, \quad i=1,2,3
$$

\section{Results}

As the numerical procedure explained here is concerned with a general two-dimensional body shape with different boundary conditions, we first validate the results of the heat conduction equation solution with the ones from the finite element analysis software COMSOL due to its capability to define analytical expression for the spatially varying thermal conductivity easily. To do so, a grid independency study is initially performed for both types of material gradation (quadratic and exponential) using four different grid sizes of $40 \times 30,80 \times 60,150 \times 100$, and $250 \times 150$. As shown in Fig. 2, the heat conducting body (the physical domain) is the area enclosed by two semicircles: the small semicircle $\left(\Gamma_{1}\right)$ of radius $5 \mathrm{~m}$ is centered at the point $(0,0) \mathrm{m}$ and the large semicircle ( $\left.\Gamma_{2}\right)$ of radius $20 \mathrm{~m}$ is centered at the point $(5,0) \mathrm{m}$. 


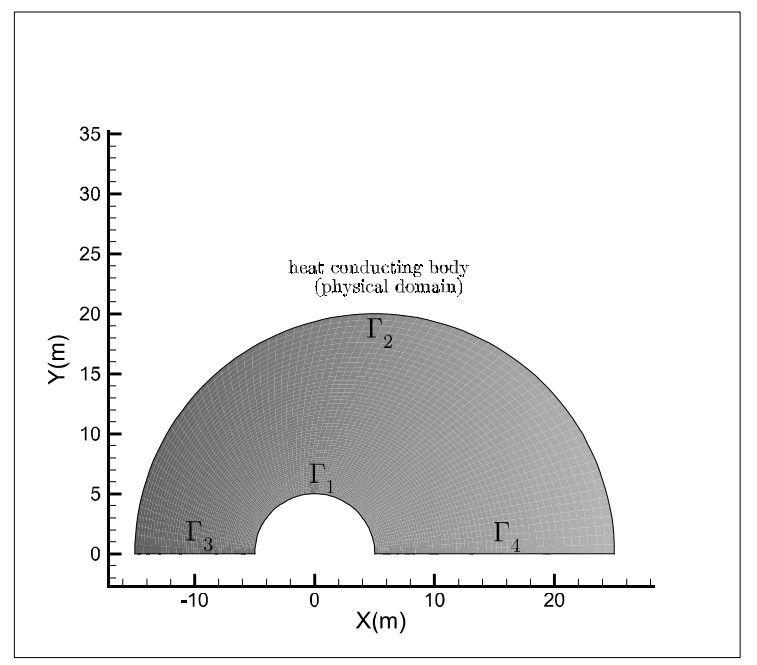

Fig. 2 Heat conducting body (FGM) used in test cases.

The numerical values of the coefficients involved in these two test cases are listed in Table 1 (for quadratic material gradation) and Table 2 (for exponential material gradation). As illustrated in Fig. 1, the heat-conducting body shown in Fig. 2 is subjected to convective heat transfer on surfaces $\Gamma_{i}, i=2,3,4$ and heat flux $\dot{q}$ on surface $\Gamma_{1}$.

Test case 1 (Quadratic material gradation):

\begin{tabular}{cccccccc}
\hline$\dot{q}\left(\frac{\mathrm{W}}{\mathrm{m}^{2}}\right)$ & $k\left(\frac{\mathrm{W}}{\mathrm{m} .{ }^{\circ} \mathrm{C}}\right)$ & $h_{2}\left(\frac{\mathrm{W}}{\mathrm{m}^{2} \cdot{ }^{\circ} \mathrm{C}}\right)$ & $h_{3}\left(\frac{\mathrm{W}}{\mathrm{m}^{2} \cdot{ }^{\circ} \mathrm{C}}\right)$ & $h_{4}\left(\frac{\mathrm{W}}{\mathrm{m}^{2} \cdot{ }^{\circ} \mathrm{C}}\right)$ & $T_{\infty_{2}}\left({ }^{\circ} \mathrm{C}\right)$ & $T_{\infty_{3}}\left({ }^{\circ} \mathrm{C}\right)$ & $T_{\infty_{4}}\left({ }^{\circ} \mathrm{C}\right)$ \\
\hline 800 & $10\left(1+0.006 x_{i, j}\right)^{2}\left(1+0.003 y_{i, j}\right)^{2}$ & 4 & 4 & 4 & 30 & 30 & 30 \\
\hline
\end{tabular}

Table 1 Data used for the test case involving quadratic material gradation.

Test case 2 (Exponential material gradation):

\begin{tabular}{cccccccc}
\hline$\dot{q}\left(\frac{\mathrm{W}}{\mathrm{m}^{2}}\right)$ & $k\left(\frac{\mathrm{W}}{\mathrm{m} .{ }^{\circ} \mathrm{C}}\right)$ & $h_{2}\left(\frac{\mathrm{W}}{\mathrm{m}^{2} \cdot{ }^{\circ} \mathrm{C}}\right)$ & $h_{3}\left(\frac{\mathrm{W}}{\mathrm{m}^{2} \cdot{ }^{\circ} \mathrm{C}}\right)$ & $h_{4}\left(\frac{\mathrm{W}}{\mathrm{m}^{2} \cdot{ }^{\circ} \mathrm{C}}\right)$ & $T_{\infty_{2}}\left({ }^{\circ} \mathrm{C}\right)$ & $T_{\infty_{3}}\left({ }^{\circ} \mathrm{C}\right)$ & $T_{\infty_{4}}\left({ }^{\circ} \mathrm{C}\right)$ \\
\hline 1200 & $6 e^{\left(1+0.025 x_{i, j}\right)} e^{\left(1+0.055 y_{i, j}\right)}$ & 3 & 3 & 3 & 20 & 20 & 20 \\
\hline
\end{tabular}

Table 2 Data used for the test case involving exponential material gradation. 
The results of the proposed numerical method are shown in Fig. 3 (for quadratic gradation type) and Fig. 6 (for exponential gradation type) and the results from the solver COMSOL for the quadratic and exponential gradation types are depicted in Fig. 4 and Fig. 7, respectively. A comparison of the results from both numerical methods for both material gradation types reveals an excellent agreement thereby confirming the correctness of the implementation and the accuracy of the proposed method. Moreover, the spatially varying thermal conductivity distribution over the body (FGM) for the quadratic and exponential gradation types are shown in Fig. 5 and Fig. 8, respectively.

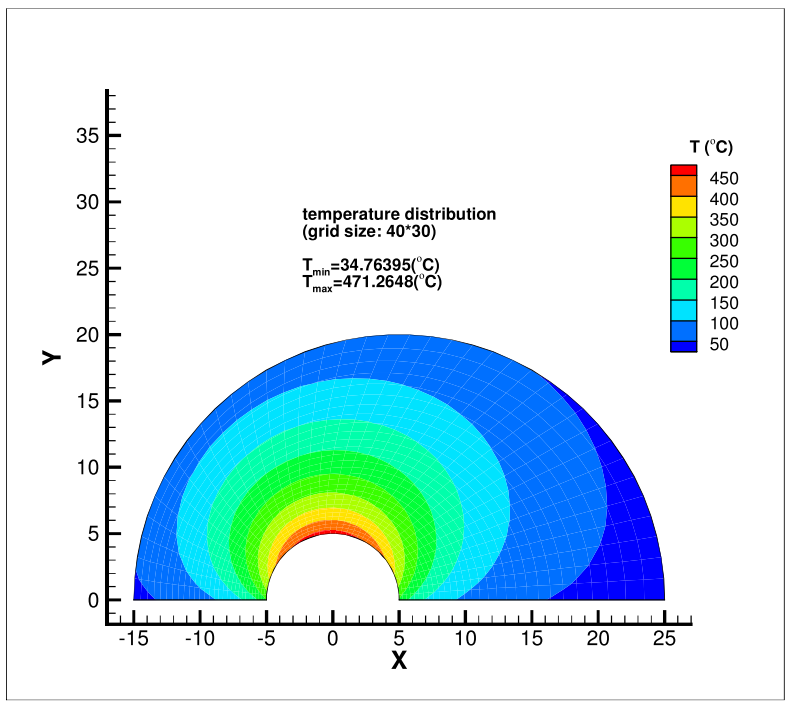

a)

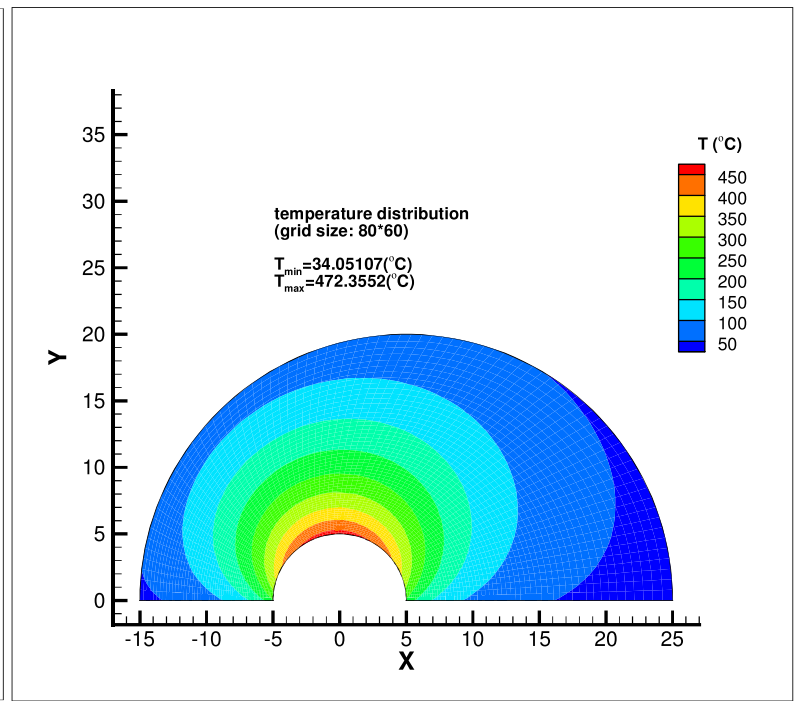

b) 


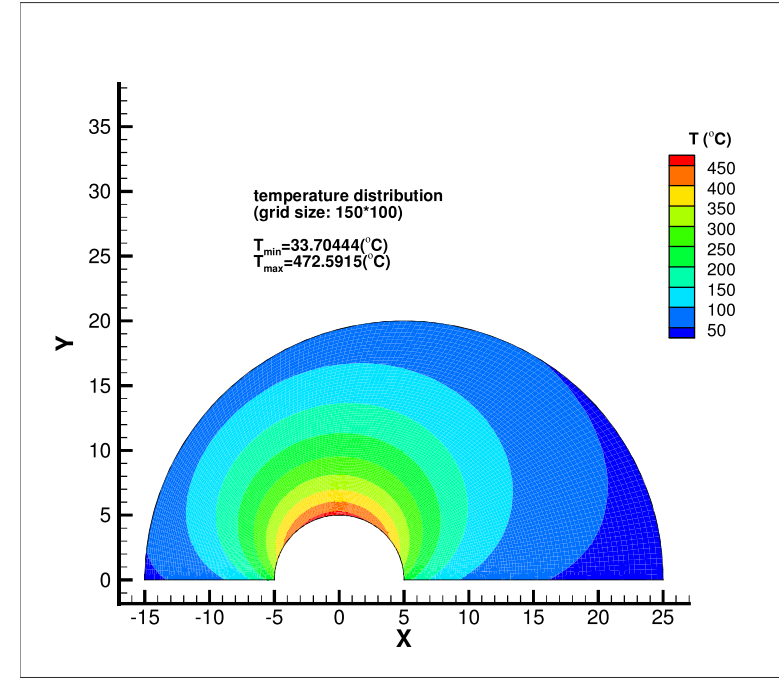

c)

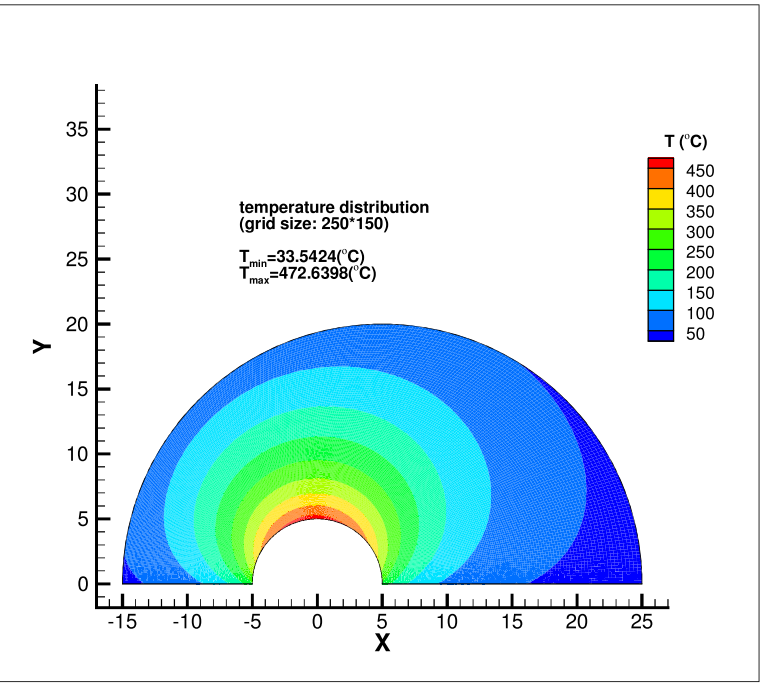

d)

Fig. 3 Grid independency study of heat conduction equation solution for quadratic material gradation. The temperature distribution using four different grid sizes of $40 \times 30$ (a), $80 \times 60$ (b), $150 \times 100$ (c), and $250 \times 150$ (d).

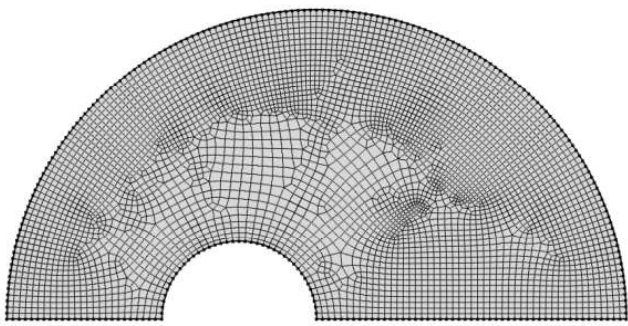

a)

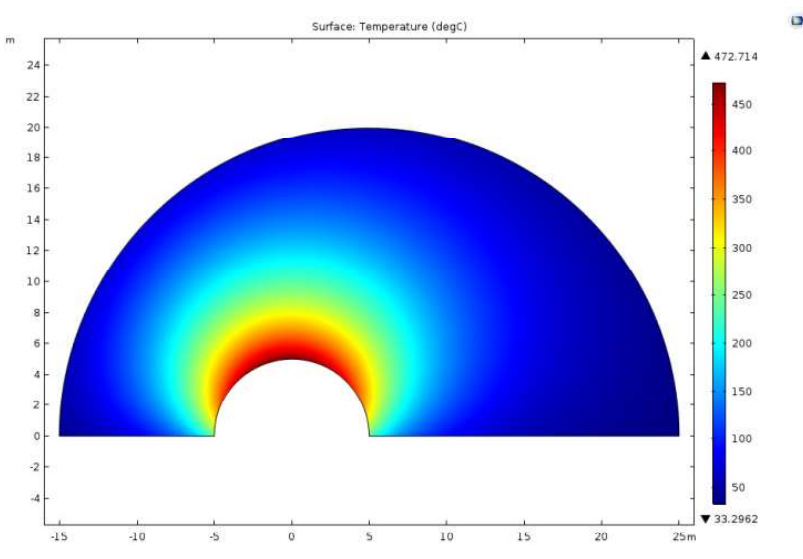

b)

Fig. 4 Solving the heat conduction equation using the finite element analysis solver COMSOL. The grid used (a) and the temperature distribution (b). 


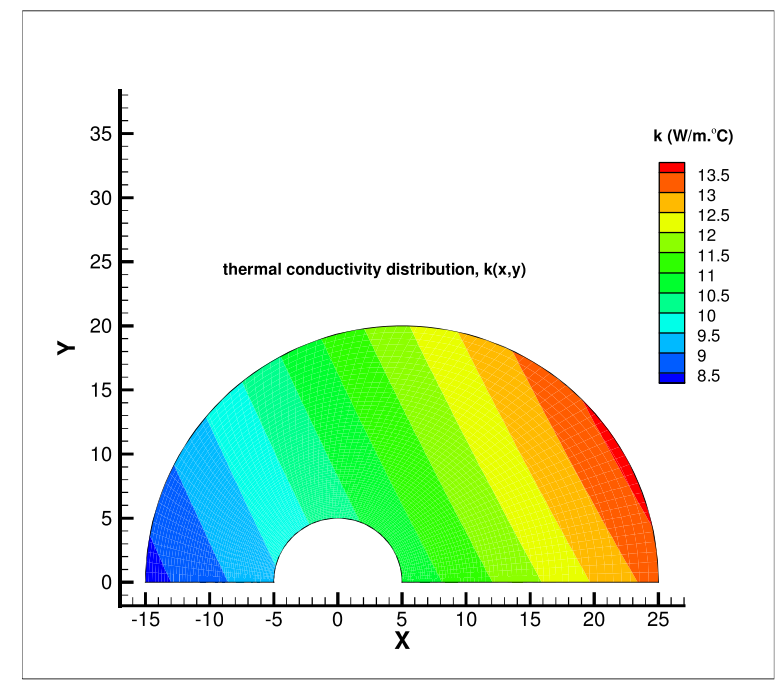

Fig. 5 Quadratic gradation of thermal conductivity.

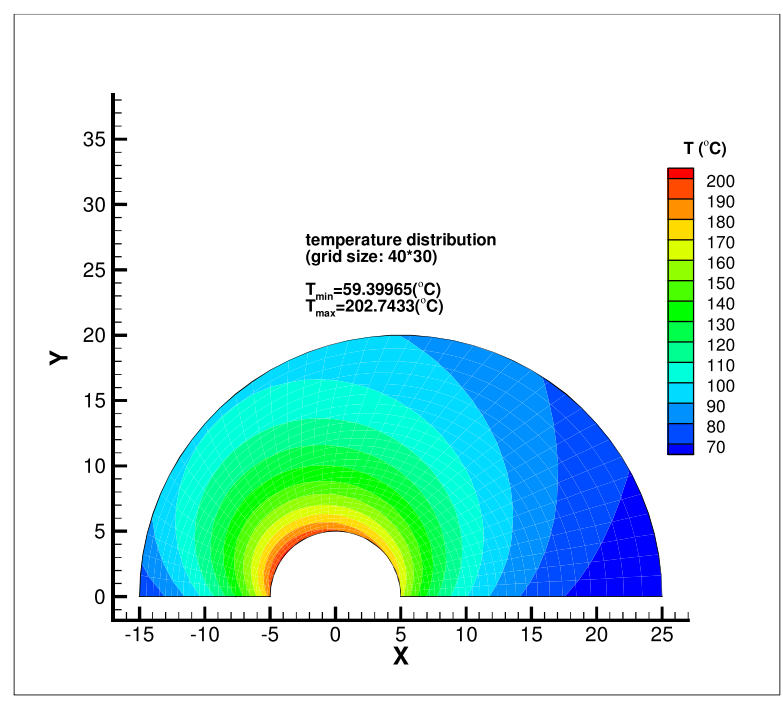

a)

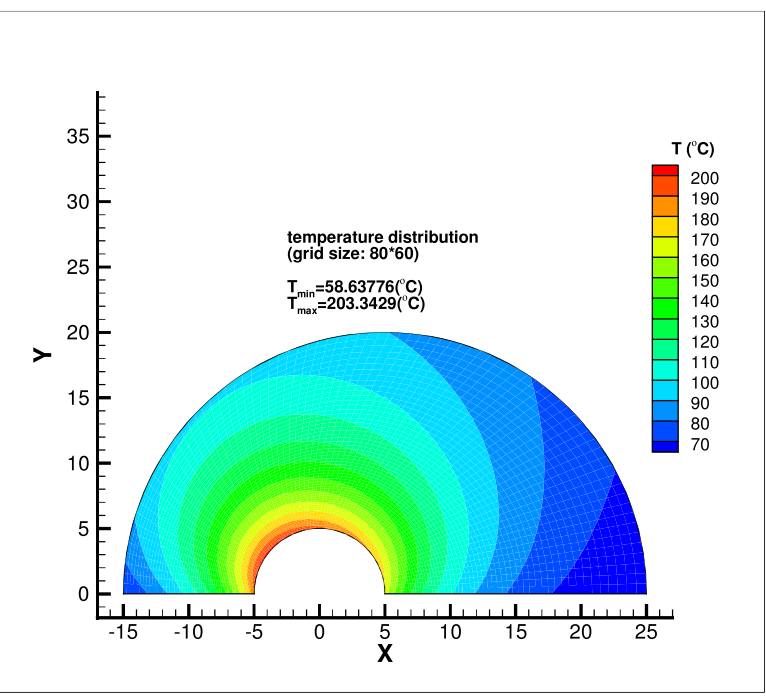

b) 


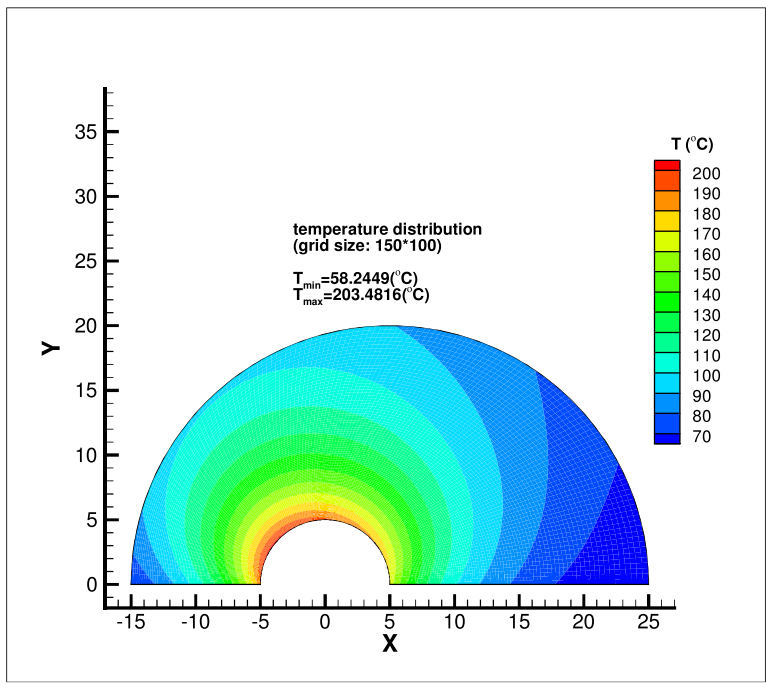

c)

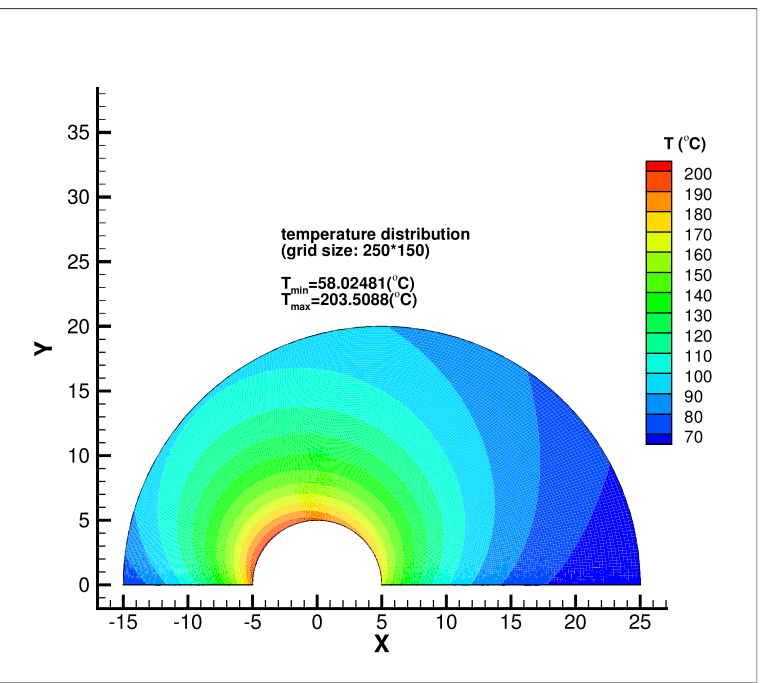

d)

Fig. 6 Grid independency study of heat conduction equation solution for exponential material gradation. The temperature distribution using four different grid sizes of $40 \times 30$ (a), $80 \times 60$ (b), $150 \times 100$ (c), and $250 \times 150$ (d).

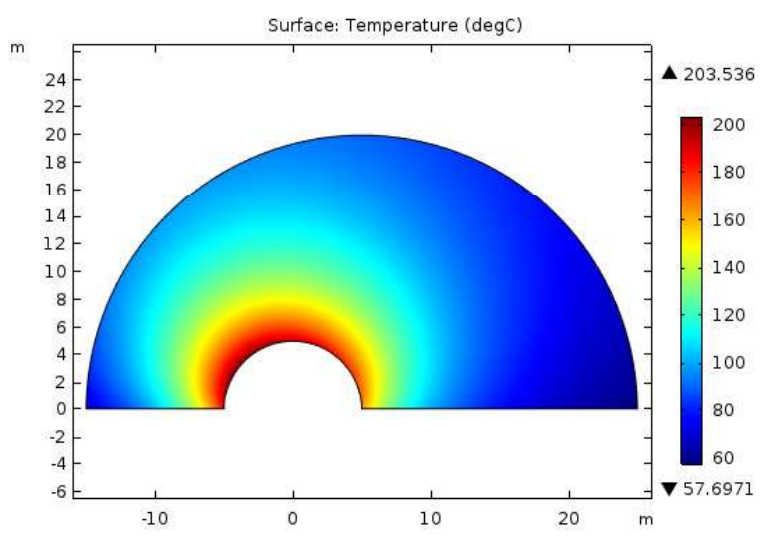

Fig. 7 Temperature distribution obtained by solving the heat conduction equation using the finite element analysis solver COMSOL. 


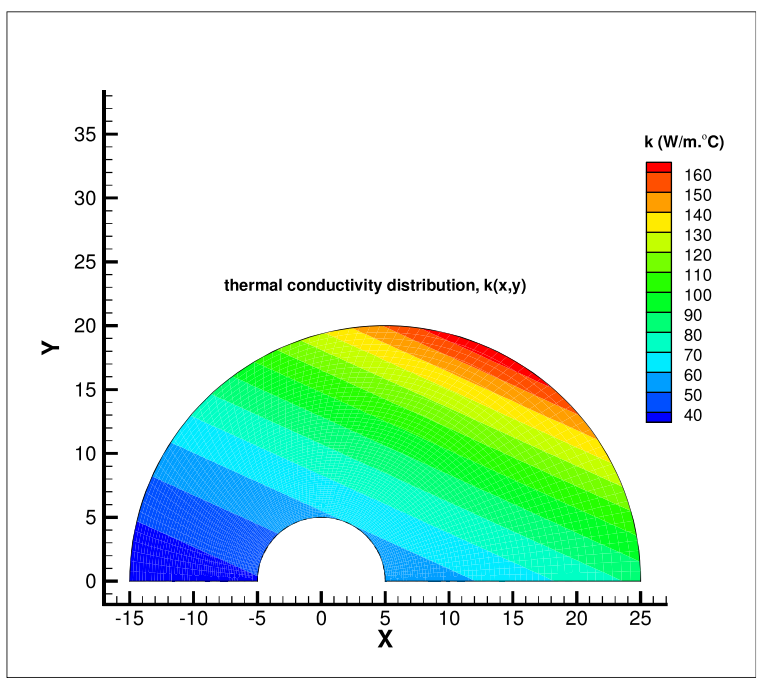

Fig. 8 Exponential gradation of thermal conductivity.

Then the computed temperature distribution $T_{i, N}(i=2, \ldots, M-1)$ is used as the simulated measured temperatures to recover the initially used spatially varying thermal conductivity through estimation of components of it, namely, $a_{l}, l=1,2,3$. In other words, the temperature distribution $T_{i, N}(i=2, \ldots, M-1)$ obtained by solving the heat conduction equation using the numerical values specified in Test cases $1 \& 2$ (Table 1 and Table 2) is used to recover $a_{1}=10, a_{2}=0.006$, and $a_{3}=0.003$ in $k=10\left(1+0.006 x_{i, j}\right)^{2}\left(1+0.003 y_{i, j}\right)^{2} \quad$ and $\quad a_{1}=6 \quad, \quad a_{2}=0.025 \quad, \quad$ and $\quad a_{3}=0.055 \quad$ in $k=6 e^{\left(1+0.025 x_{i, j}\right)} e^{\left(1+0.055 y_{i, j}\right)}$, respectively. To facilitate the computation of the sensitivity matrix coefficients using the central finite-difference relations, the grid nodes $(1, N)$ and $(M, N)$ on corners of the outer surface $\Gamma_{2}$ are excluded from computing the temperature distribution. In the inverse analysis, the square of the difference between the temperature distribution of the outer surface $\Gamma_{2}$ (obtained from the solution the direct problem at each iteration) and the simulated measured temperature distribution of the same surface $\left(\Gamma_{2}\right)$ is to be minimized.

Test case 1: Using the problem data given in Table 1 (for quadratic gradation type), the known (desired) values of $a_{1_{d}}=10.0, a_{2_{d}}=0.006$, and $a_{3_{d}}=0.003$ are to be 
recovered by an inverse analysis using three different initial guesses to demonstrate the robustness of the inverse analysis:

$$
\begin{aligned}
& a_{1_{\text {initial }_{1}}}=25.0, \quad a_{2_{\text {initial }_{1}}}=0.05, \quad a_{3_{\text {initial }_{1}}}=0.001 \\
& a_{1_{\text {initial }_{2}}}=2.0, \quad a_{2_{\text {initial }_{2}}}=0.0001, \quad a_{3_{\text {initial }_{2}}}=0.1 \\
& a_{1_{\text {initial }_{3}}}=300.0, \quad a_{2_{\text {initial }_{3}}}=0.0, \quad a_{3_{\text {initial }_{3}}}=0.0
\end{aligned}
$$

Initial guess 1: $a_{1_{\text {initial }_{1}}}=25.0, \quad a_{2_{\text {initial }_{1}}}=0.05, \quad a_{3_{\text {initial }_{1}}}=0.001$

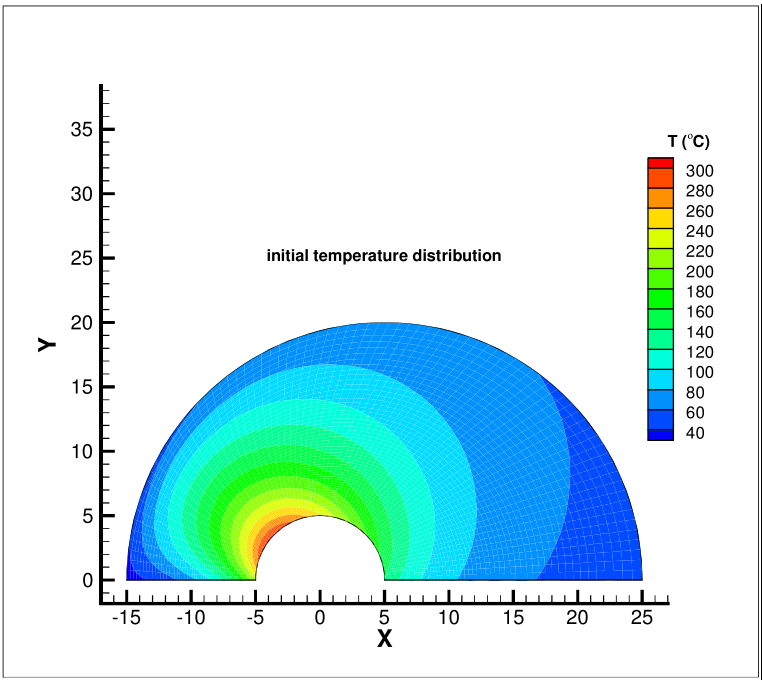

a)

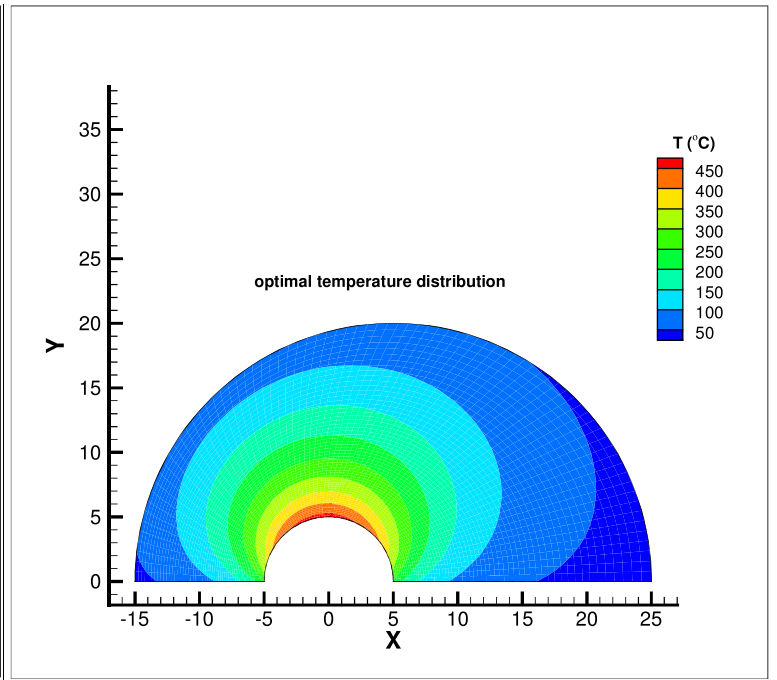

b)

Fig. 9 Initial (a) and optimal (b) temperature distribution. 


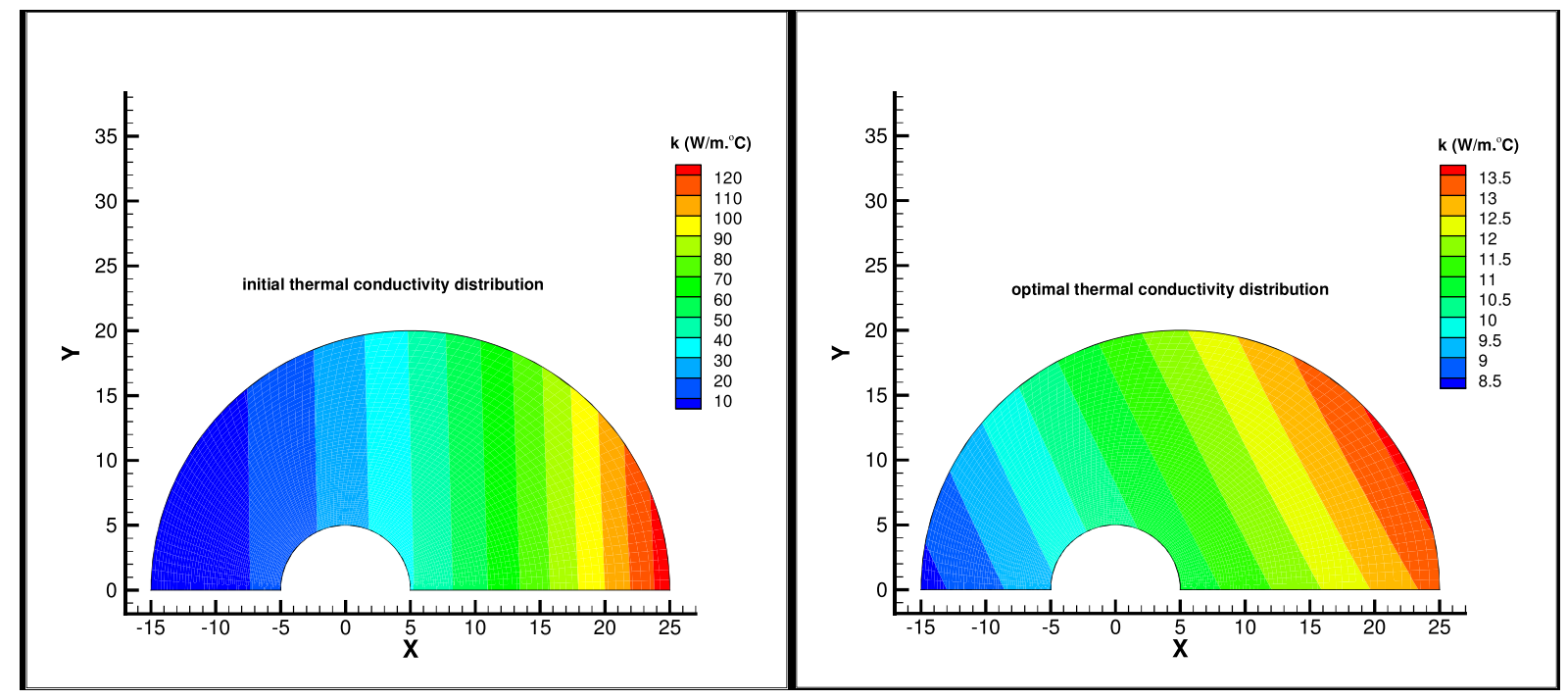

a)

b)

Fig. 10 Initial (a) and optimal (b) thermal conductivity distribution.

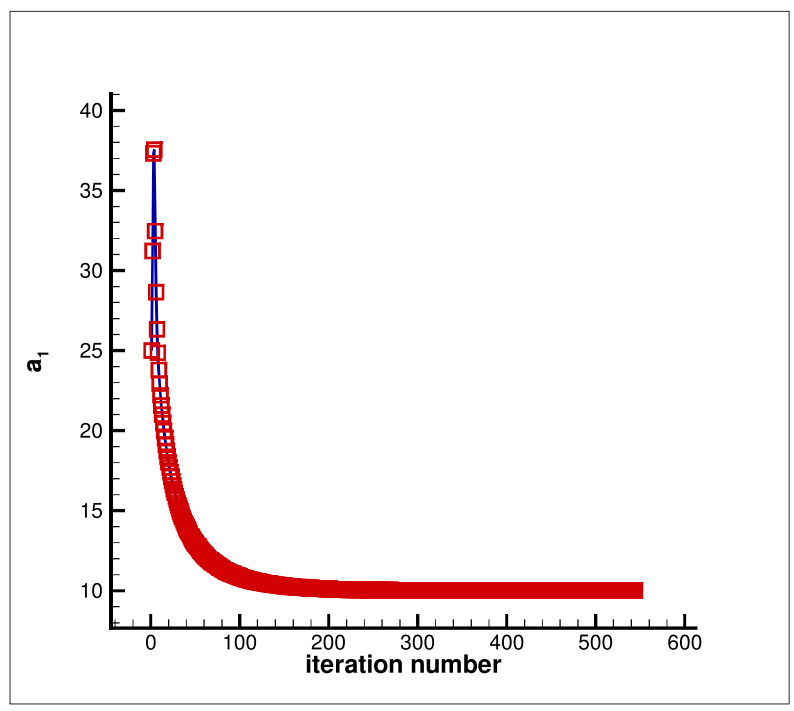

a)

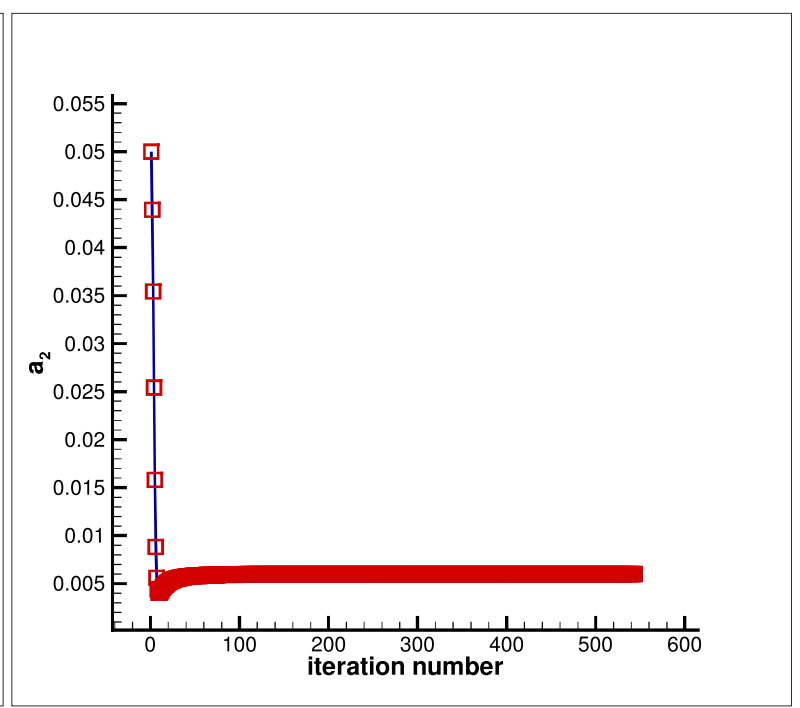

b) 


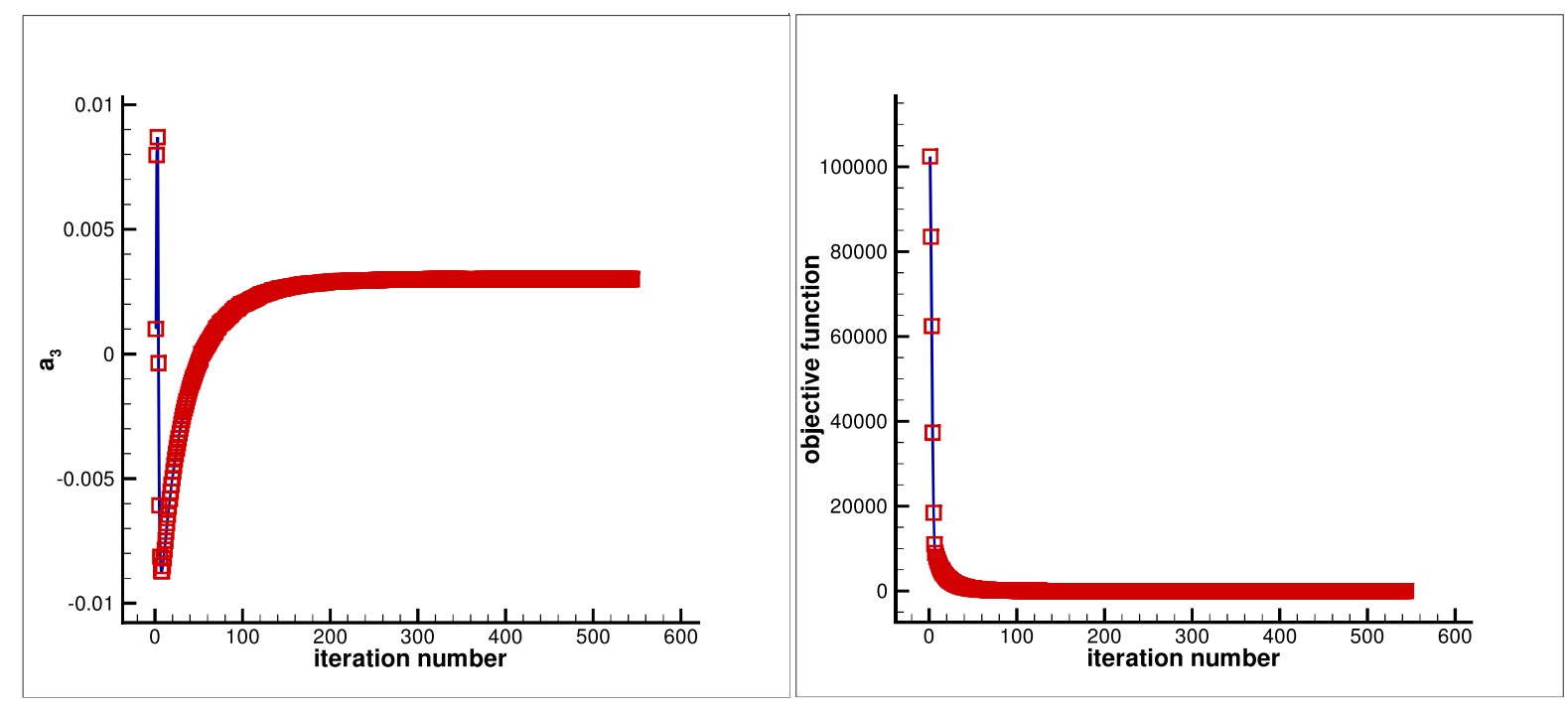

c)

d)

Fig. 11 Estimation of $a_{1}, a_{2}, a_{3}\left(k=a_{1}\left(1+a_{2} x\right)^{2}\left(1+a_{3} y\right)^{2}\right)(\mathrm{a}-\mathrm{c})$ and objective function versus iteration number (d) for initial thermal conductivity $k_{\text {initial }_{1}}=25\left(1+0.05 x_{i, j}\right)^{2}\left(1+0.001 y_{i, j}\right)^{2}\left(\frac{\mathrm{W}}{\mathrm{m} .{ }^{\circ} \mathrm{C}}\right)$.

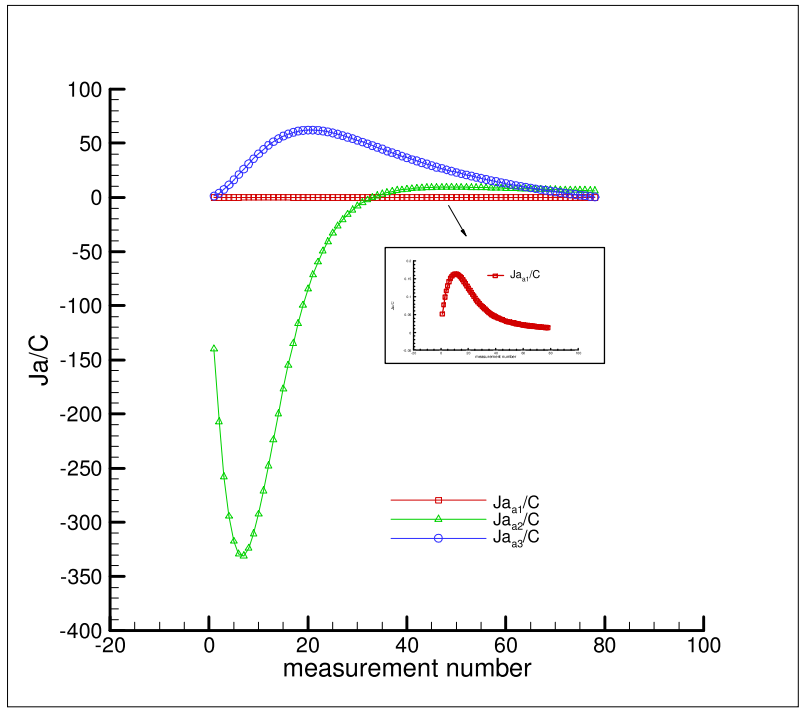

Fig. 12 Sensitivity coefficients given by Eq. (37). 
Initial guess 2: $a_{1_{\text {initial }_{2}}}=2.0, \quad a_{2_{\text {initial }_{2}}}=0.0001, \quad a_{3_{\text {initial }_{2}}}=0.1$

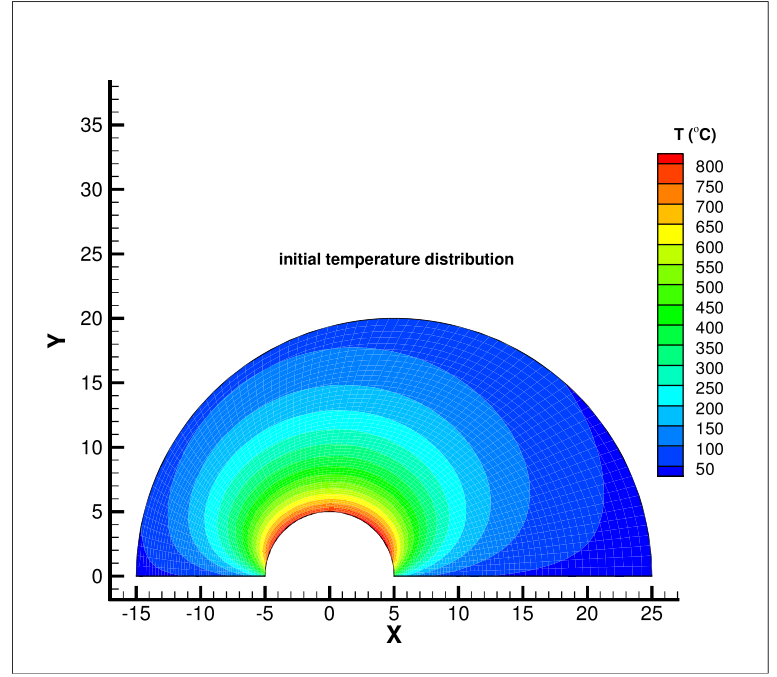

a)

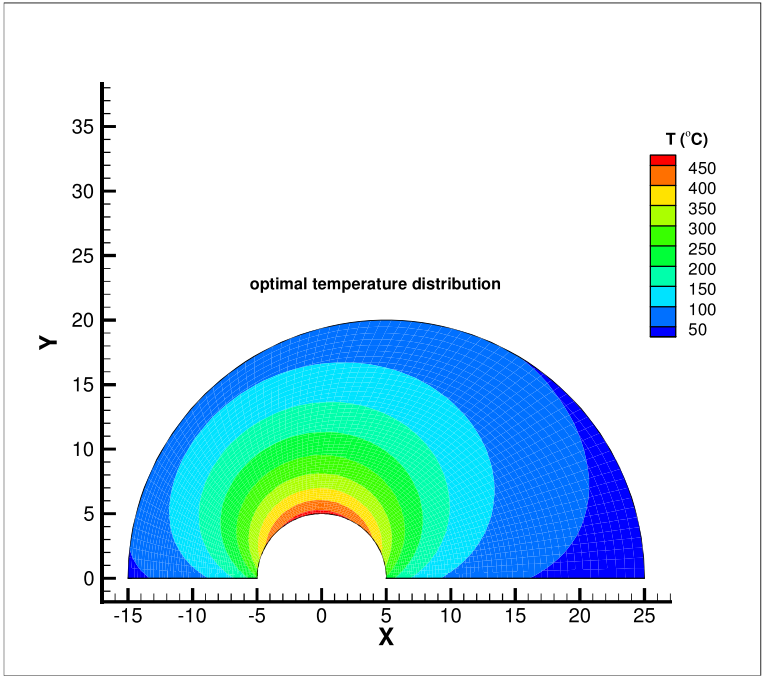

b)

Fig. 13 Initial (a) and optimal (b) temperature distribution.

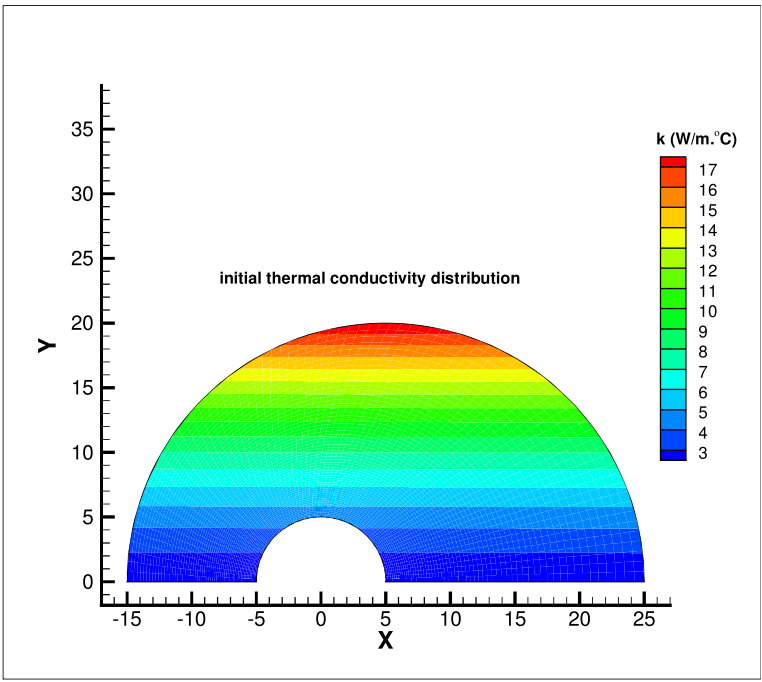

a)

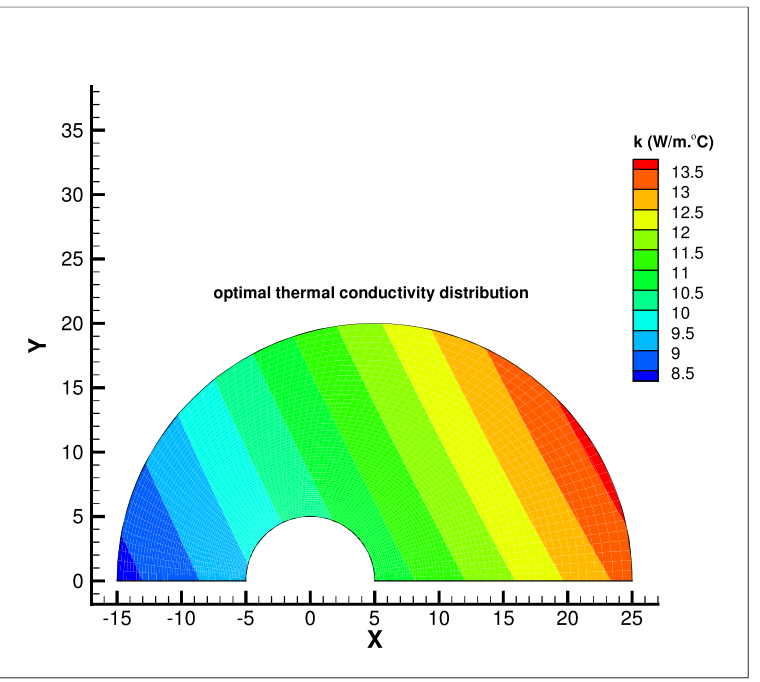

b)

Fig. 14 Initial (a) and optimal (b) thermal conductivity distribution. 


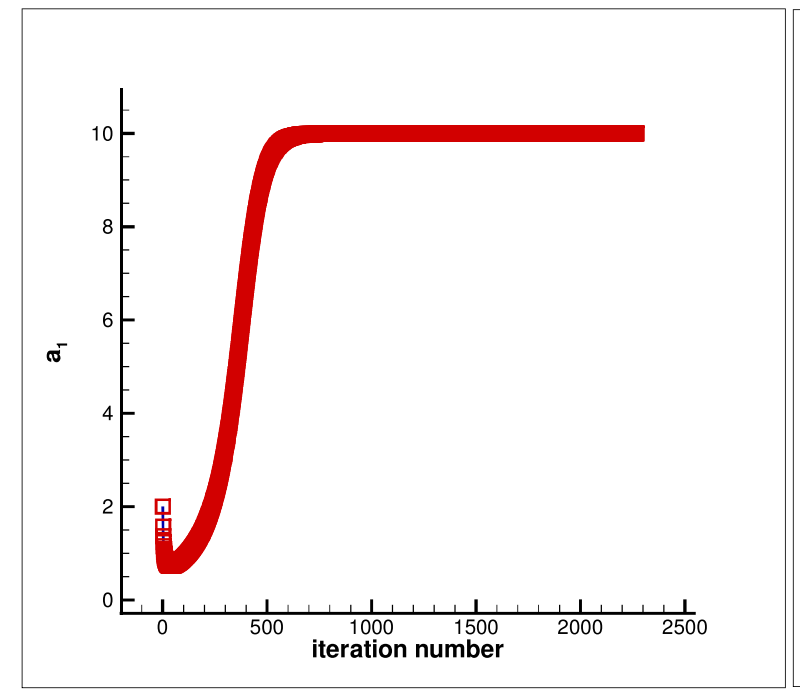

a)

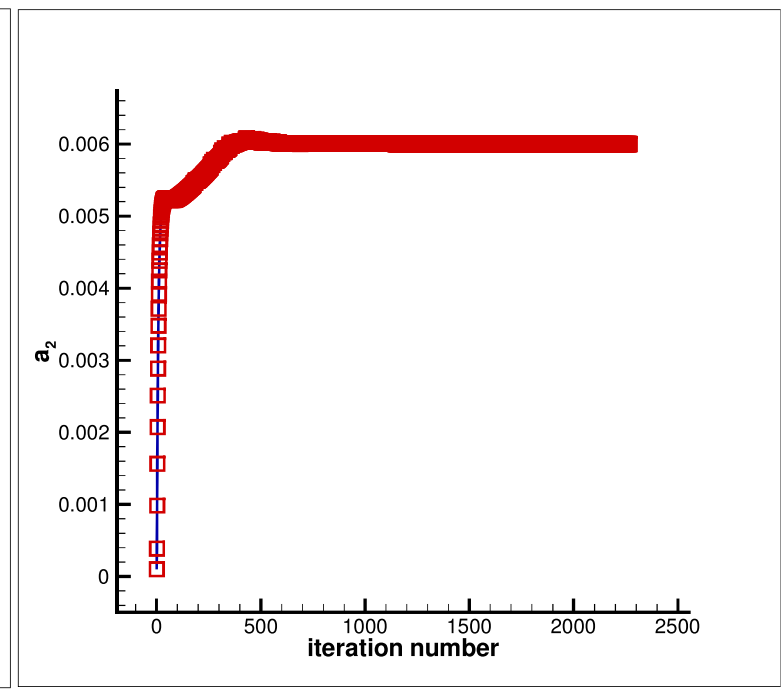

b)

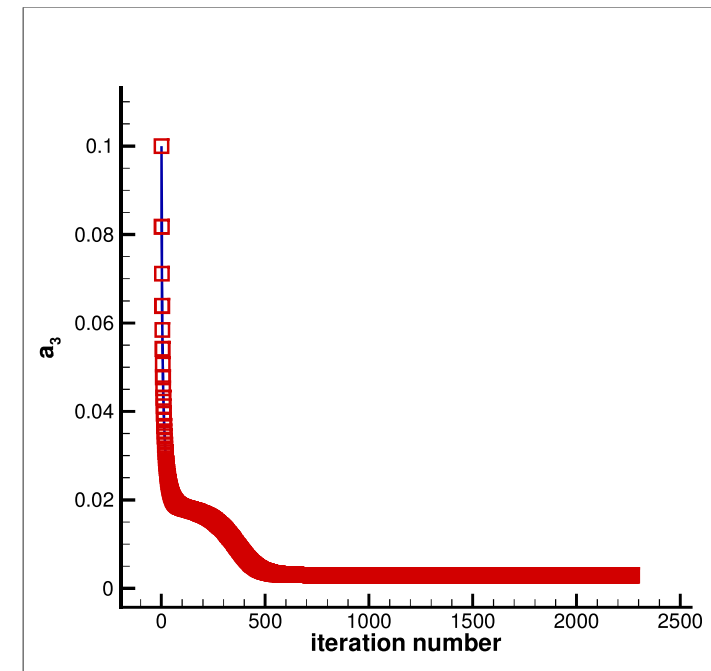

c)

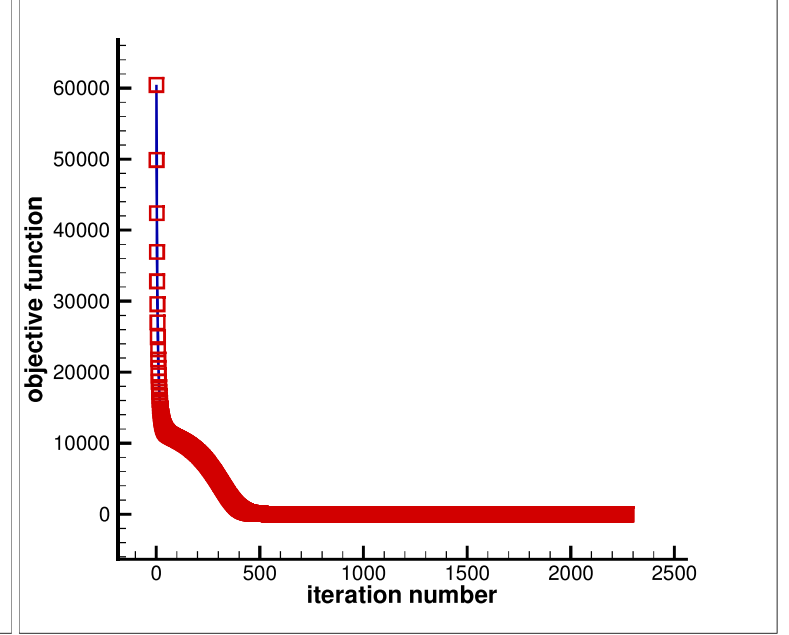

d)

Fig. 15 Estimation of $a_{1}, a_{2}, a_{3}\left(k=a_{1}\left(1+a_{2} x\right)^{2}\left(1+a_{3} y\right)^{2}\right)(\mathrm{a}-\mathrm{c})$ and objective function versus iteration number (d) for initial thermal conductivity $k_{\text {initial }_{2}}=2\left(1+0.0001 x_{i, j}\right)^{2}\left(1+0.1 y_{i, j}\right)^{2}\left(\frac{\mathrm{W}}{\mathrm{m} .{ }^{\circ} \mathrm{C}}\right)$. 


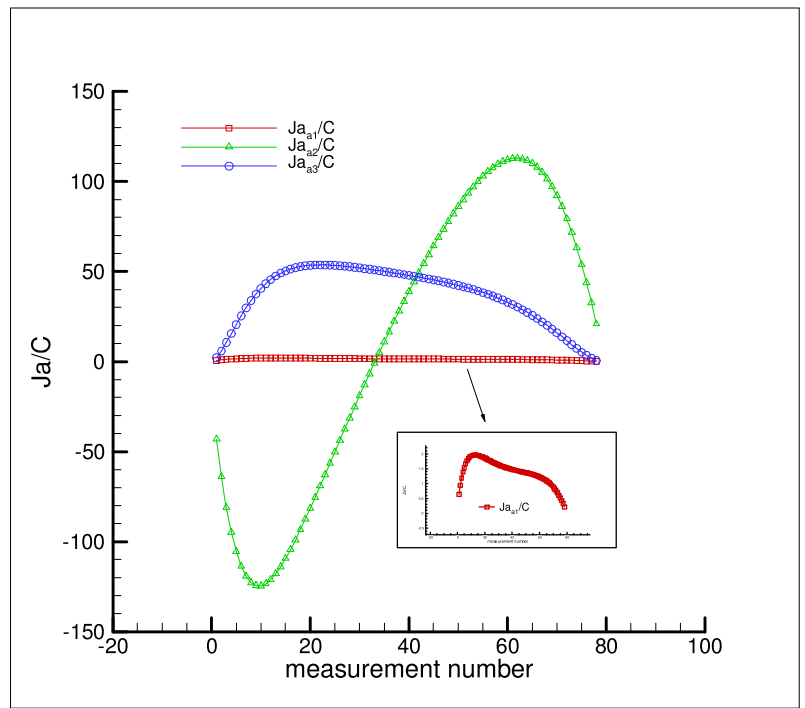

Fig. 16 Sensitivity coefficients given by Eq. (37).

Initial guess 3: $a_{1_{\text {initial }_{3}}}=300.0, \quad a_{2_{\text {initial }_{3}}}=0.0, \quad a_{3_{\text {initial }_{3}}}=0.0$

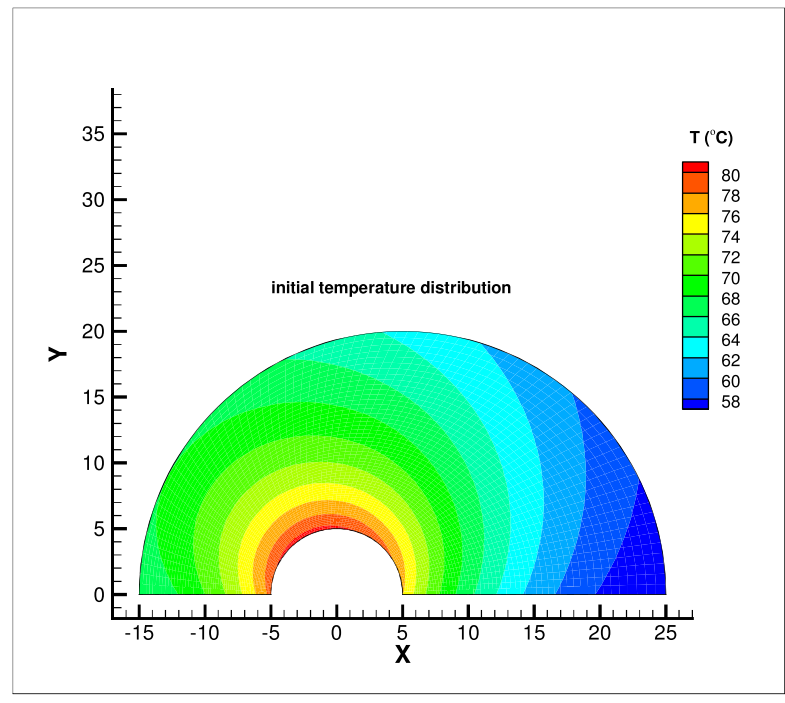

a)

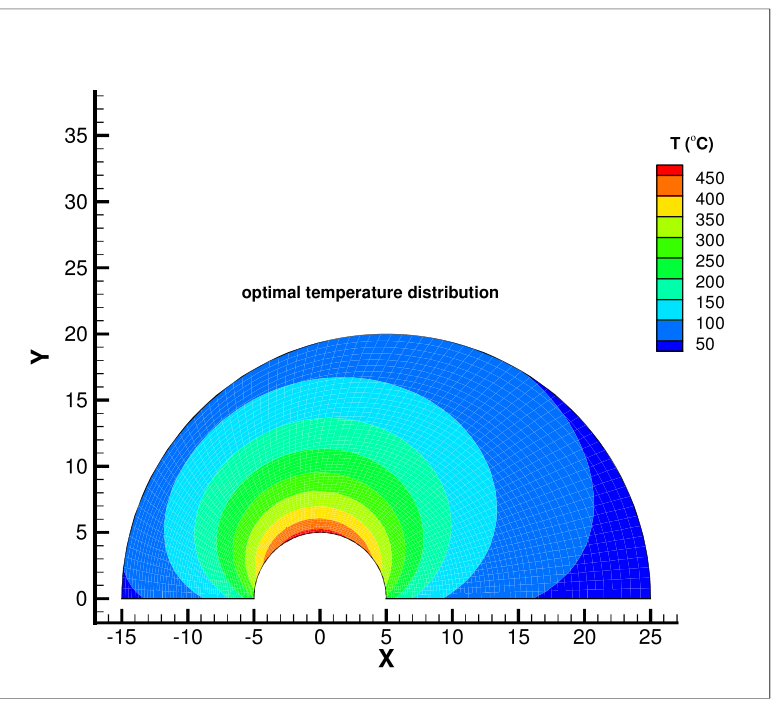

b)

Fig. 17 Initial (a) and optimal (b) temperature distribution. 


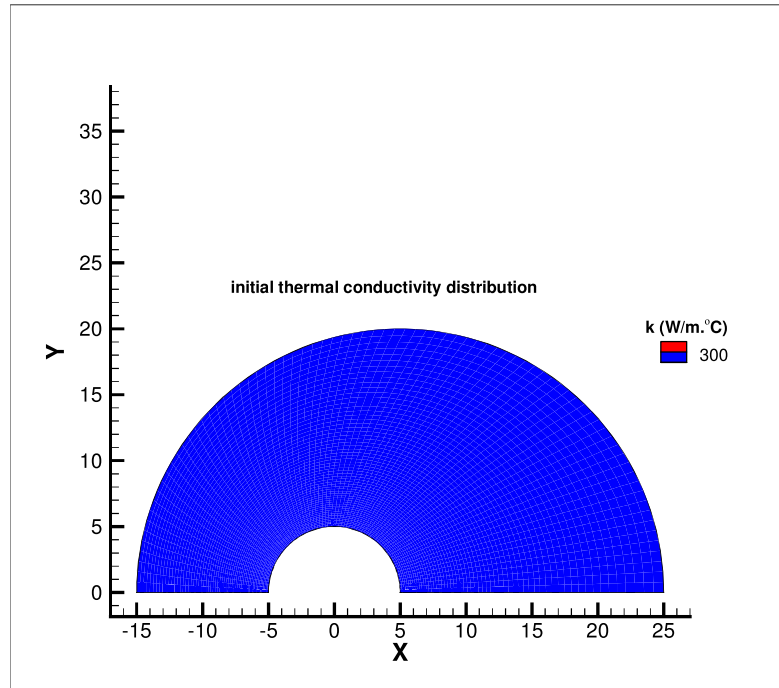

a)

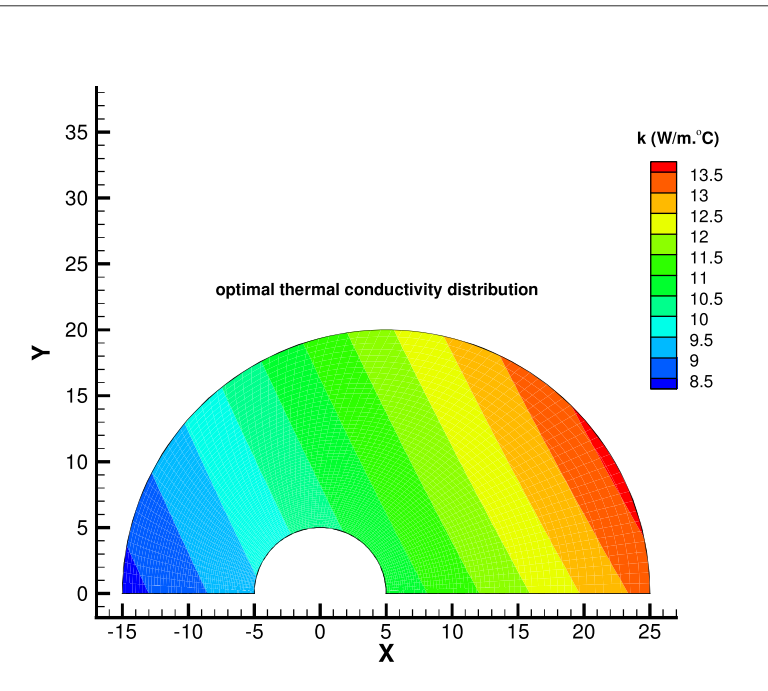

b)

Fig. 18 Initial (a) and optimal (b) thermal conductivity distribution.

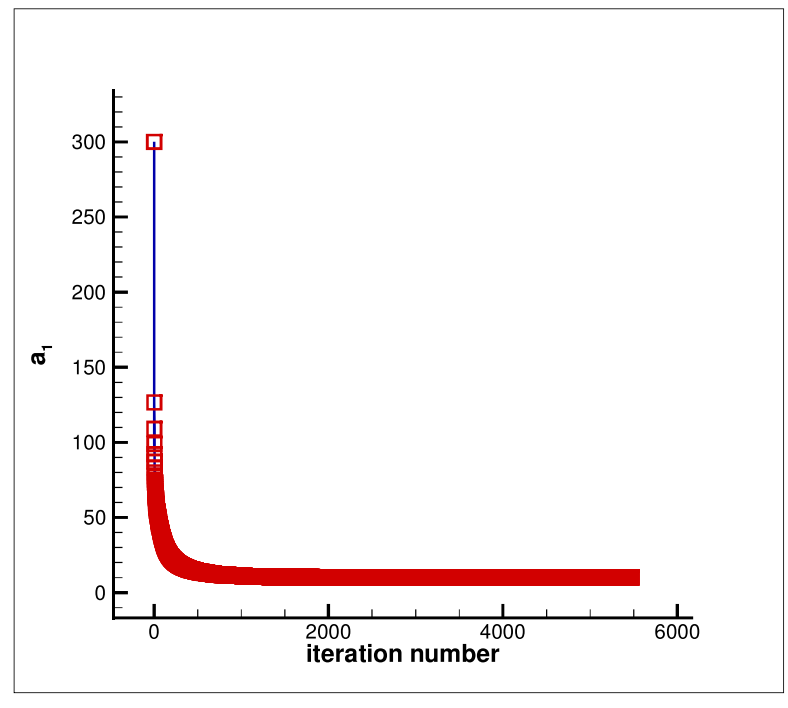

a)

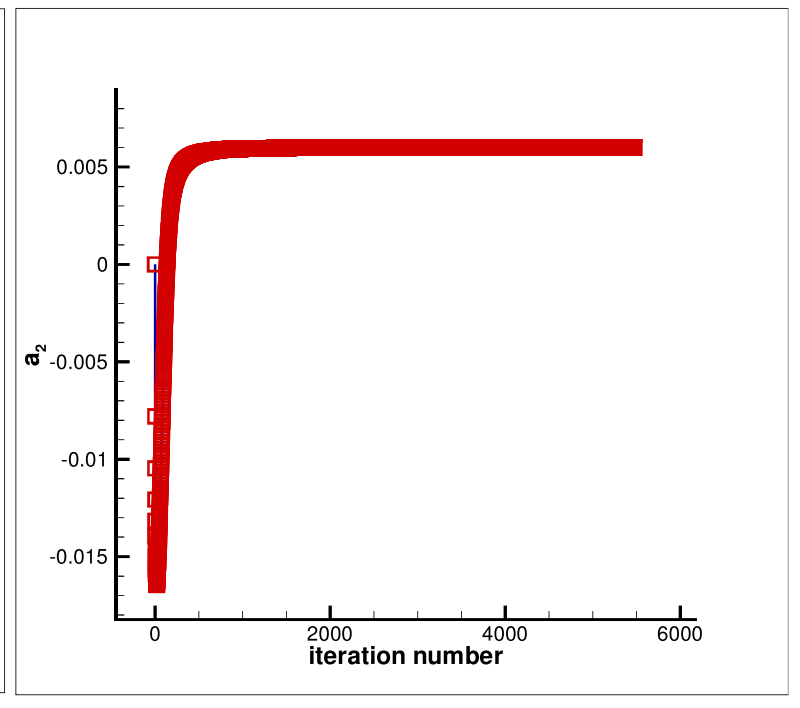

b) 


$$
\text { I }
$$


Initial guess 1 (with measurement error): $a_{1_{\text {initial }_{1}}}=25.0, \quad a_{2_{\text {initial }_{1}}}=0.05, \quad a_{3_{\text {initial }_{1}}}=0.001$

In this study, the measured temperature containing random errors, $T_{i, N}^{\text {meas }}$ $(i=2, \ldots, M-1)$, is generated by adding an error term $\omega \sigma$ to the exact temperature $T_{i, N}^{\text {exact }}$ to give

$$
T_{i, N}^{\text {meas }}=T_{i, N}^{\text {exact }}+\omega \sigma
$$

where $\omega$ is a random variable with normal distribution, zero mean, and unitary standard deviation. Assuming $99 \%$ confidence for the measured temperature, $\omega$ lies in the range $-2.576 \leq \omega \leq 2.576$ and it is randomly generated by using MATLAB. $\sigma$ is the standard deviation of the measurement errors. In this study, for both test cases $\sigma=0.5$. The first initial guess, $a_{1_{\text {initial }_{1}}}=25.0, a_{2_{\text {initial }_{1}}}=0.05, a_{3_{\text {initial }_{1}}}=0.001$ is considered to initiate the optimization process.

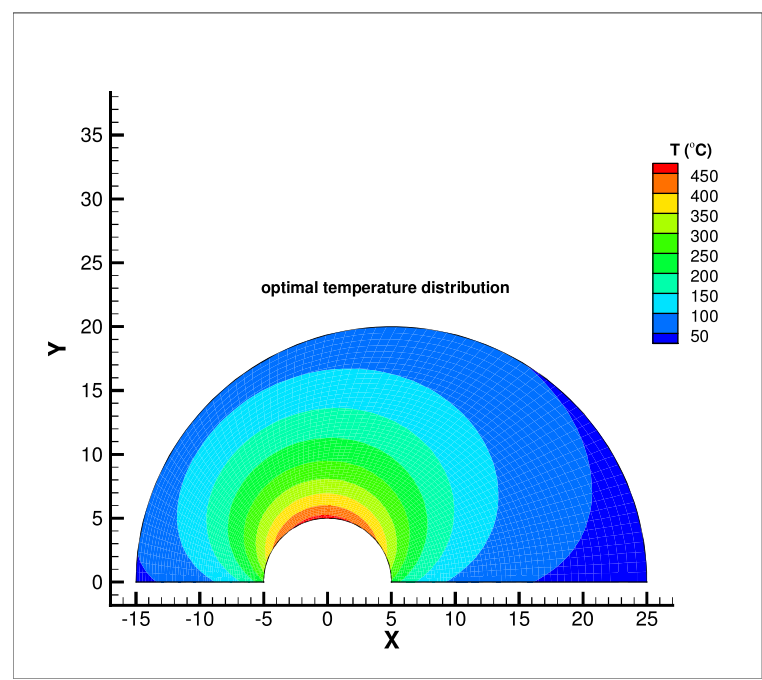

a)

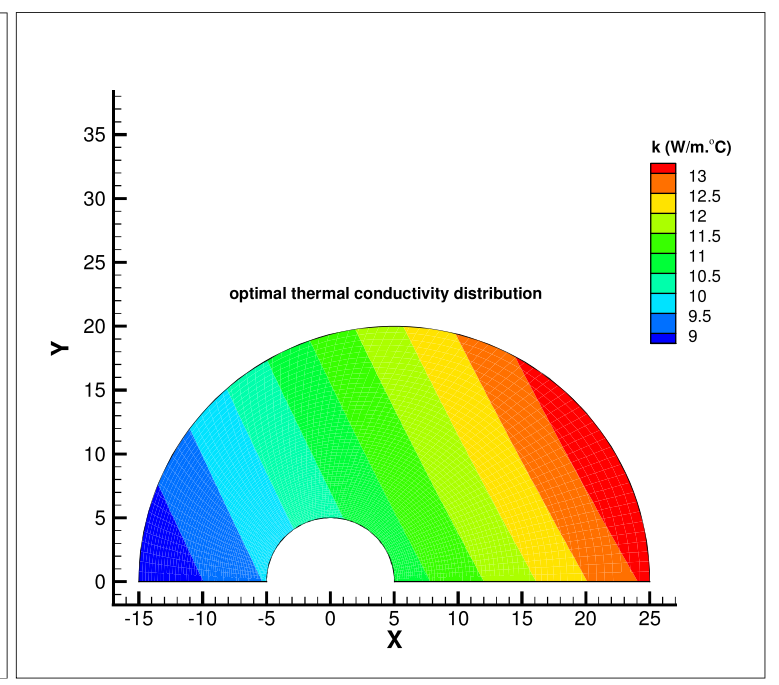

b)

Fig. 21 Optimal temperature distribution (a) and thermal conductivity (b). 


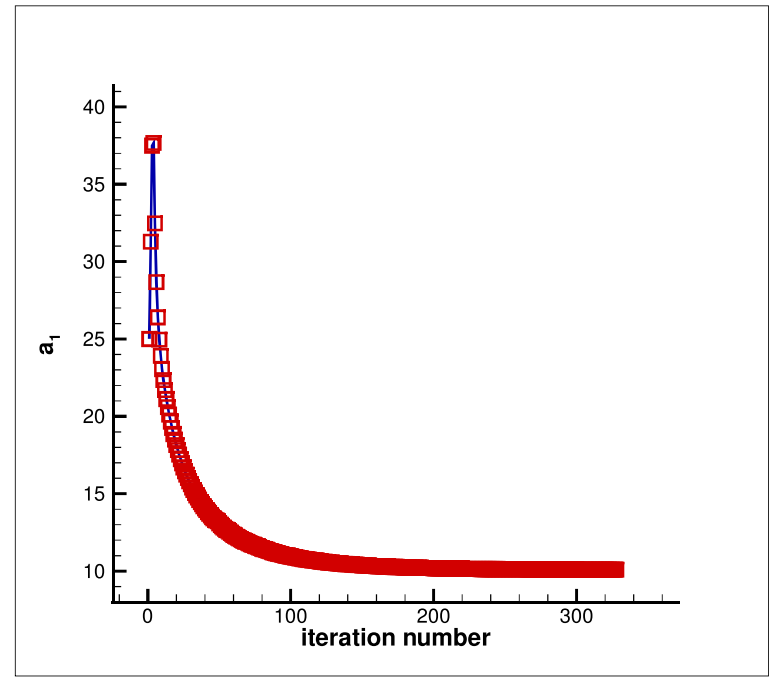

a)

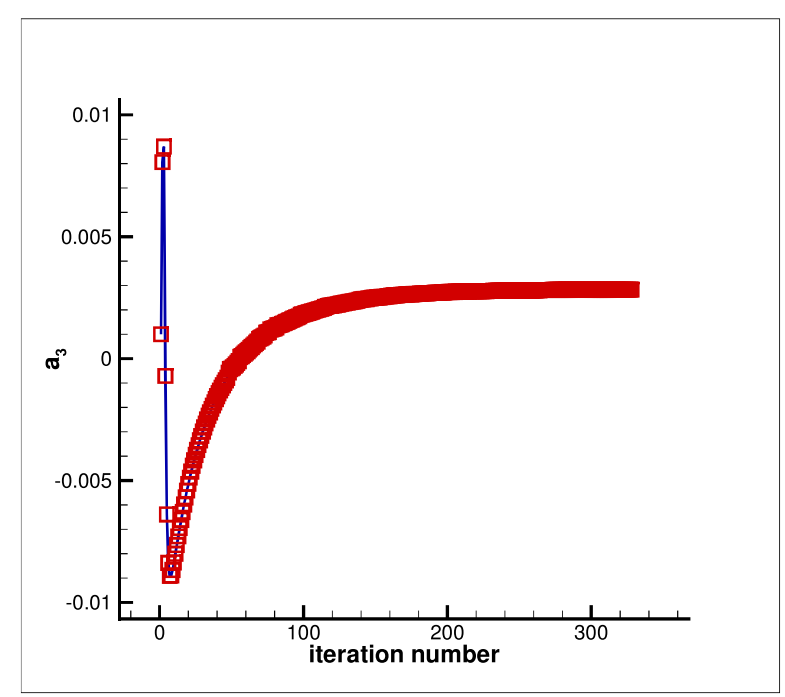

c)

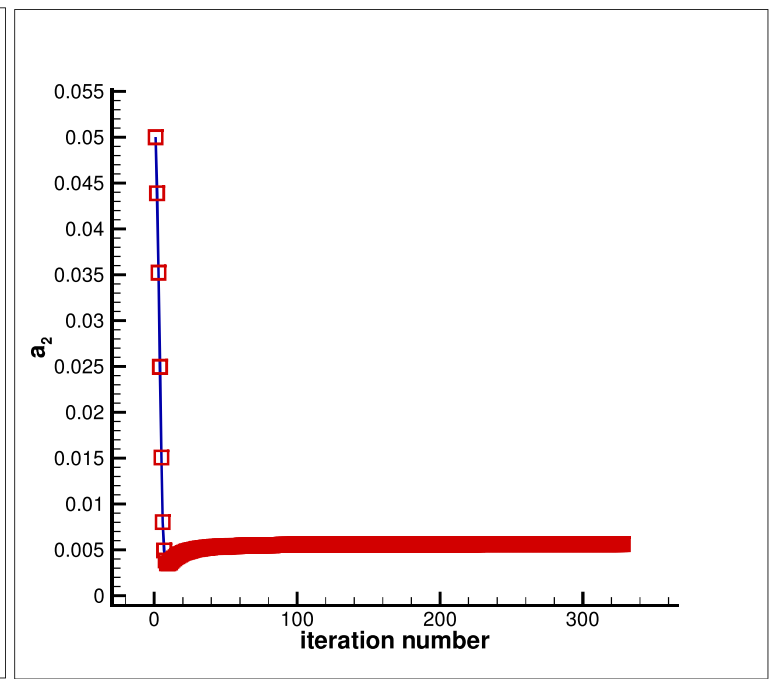

b)

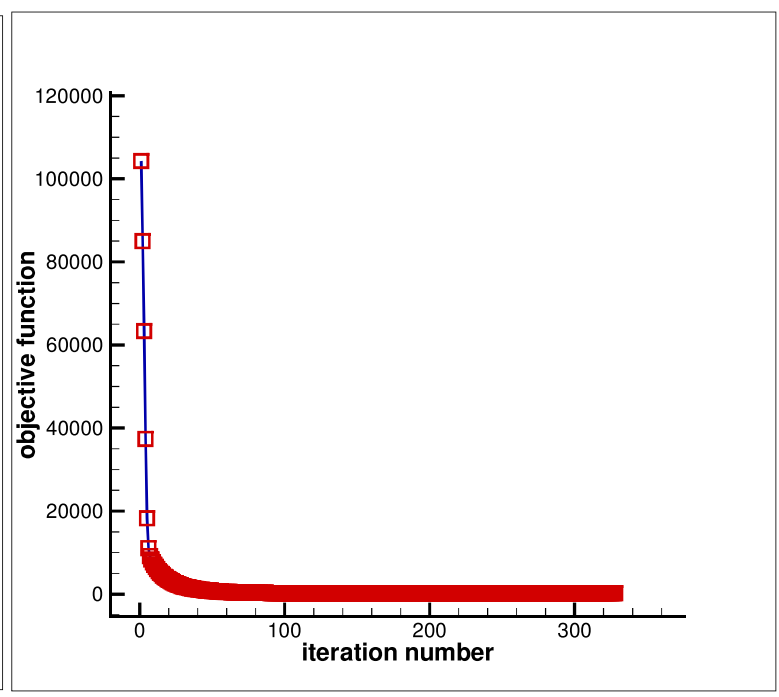

d)

Fig. 22 Estimation of $a_{1}, a_{2}, a_{3}\left(k=a_{1}\left(1+a_{2} x\right)^{2}\left(1+a_{3} y\right)^{2}\right)$ (a-c) and objective function versus iteration number (d) for initial thermal conductivity $k_{\text {initial }_{1}}=25\left(1+0.05 x_{i, j}\right)^{2}\left(1+0.001 y_{i, j}\right)^{2}\left(\frac{\mathrm{W}}{\mathrm{m} .{ }^{\circ} \mathrm{C}}\right)$ by considering measurement error $(\sigma=0.5)$. 


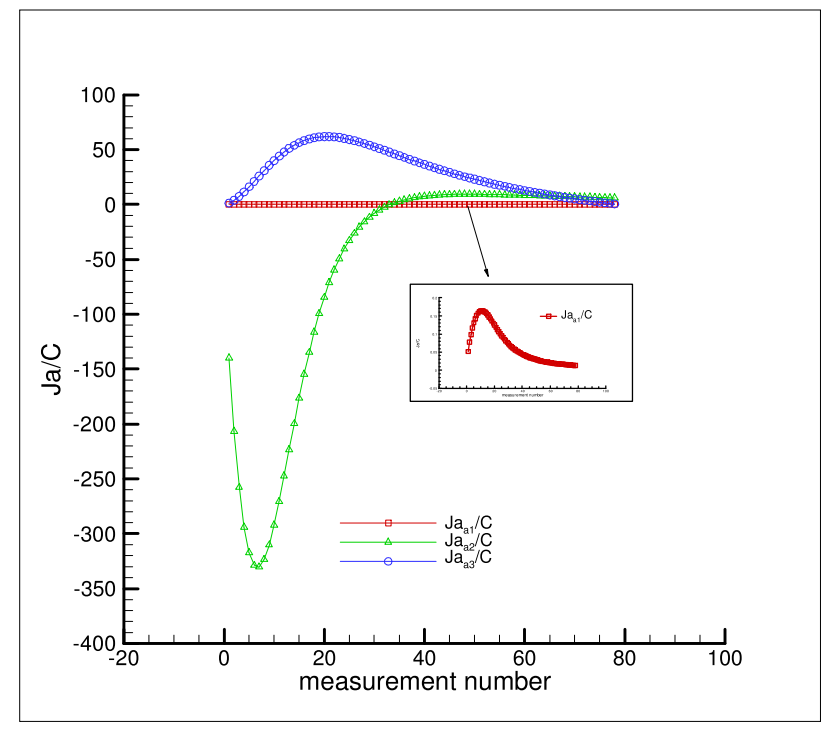

Fig. 23 Sensitivity coefficients given by Eq. (37).

Test case 2: Using the problem data given in Table 2 (for exponential gradation type), the known (desired) values of $a_{1_{d}}=6.0, a_{2_{d}}=0.025$, and $a_{3_{d}}=0.055$ are to be recovered by an inverse analysis using three different initial guesses:

$$
\begin{aligned}
& a_{1_{\text {initial }_{1}}}=3.0, \quad a_{2_{\text {initial }_{1}}}=0.01, \quad a_{3_{\text {initial }_{1}}}=0.0001 \\
& a_{1_{\text {initial }_{2}}}=25.0, \quad a_{2_{\text {initial }_{2}}}=0.00001, \quad a_{3_{\text {initial }_{2}}}=0.00001 \\
& a_{1_{\text {initial }_{3}}}=50.0, \quad a_{2_{\text {initial }_{3}}}=0.0, \quad a_{3_{\text {initial }_{3}}}=0.0
\end{aligned}
$$


Initial guess 1: $a_{1_{\text {initial }_{1}}}=3.0, \quad a_{2_{\text {initial }_{1}}}=0.01, \quad a_{3_{\text {initial }_{1}}}=0.0001$

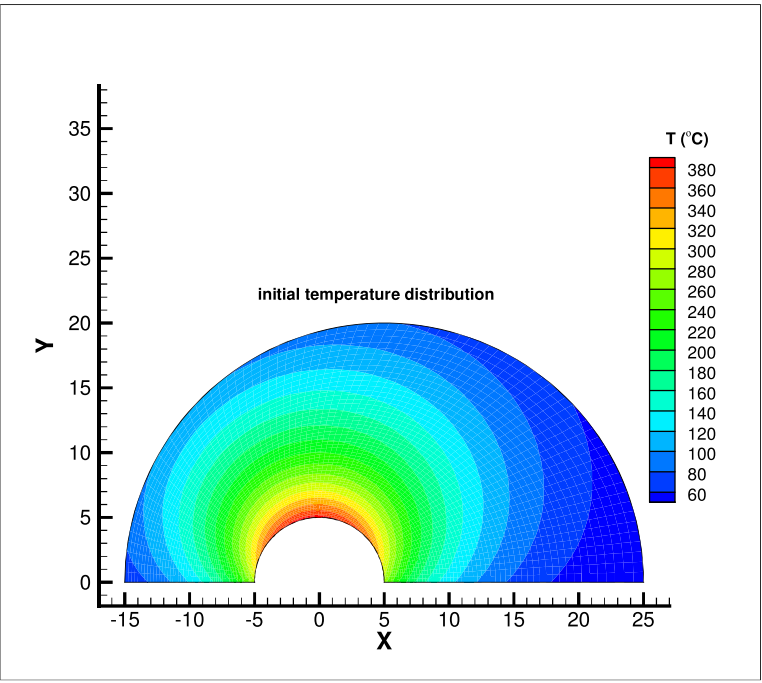

a)

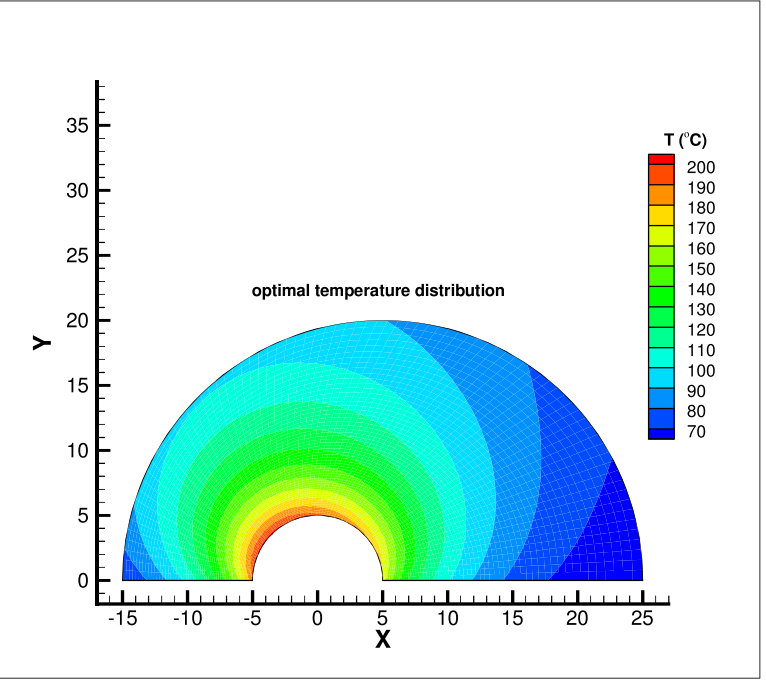

b)

Fig. 24 Initial (a) and optimal (b) temperature distribution.

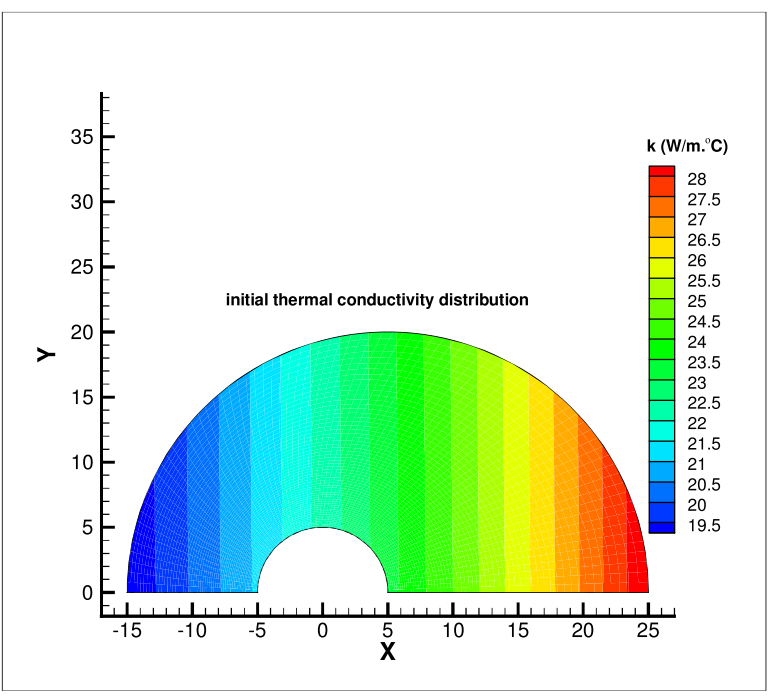

a)

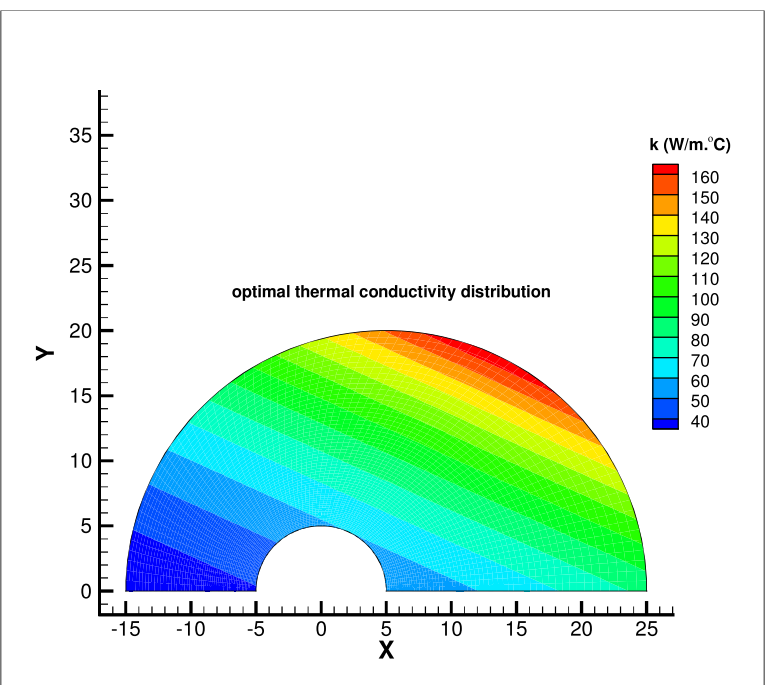

b)

Fig. 25 Initial (a) and optimal (b) thermal conductivity distribution. 


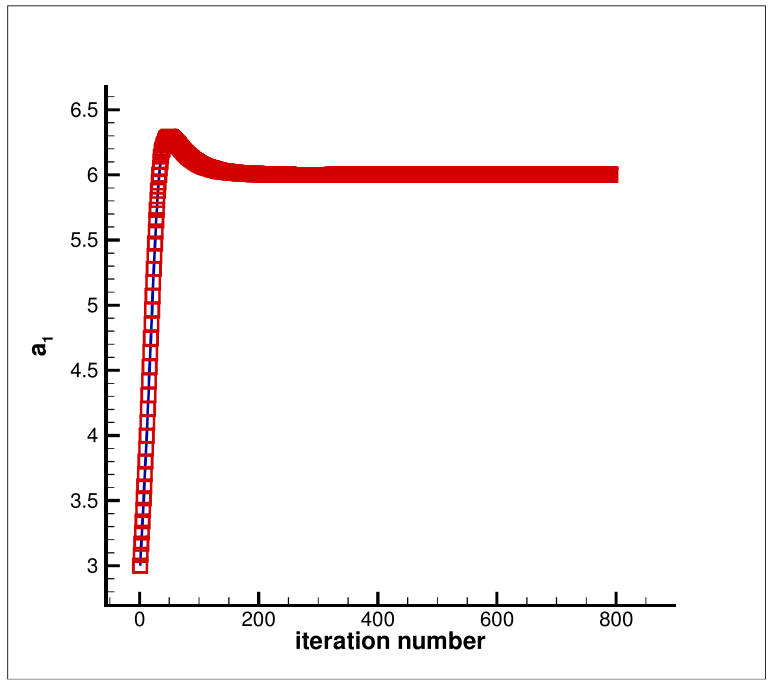

a)

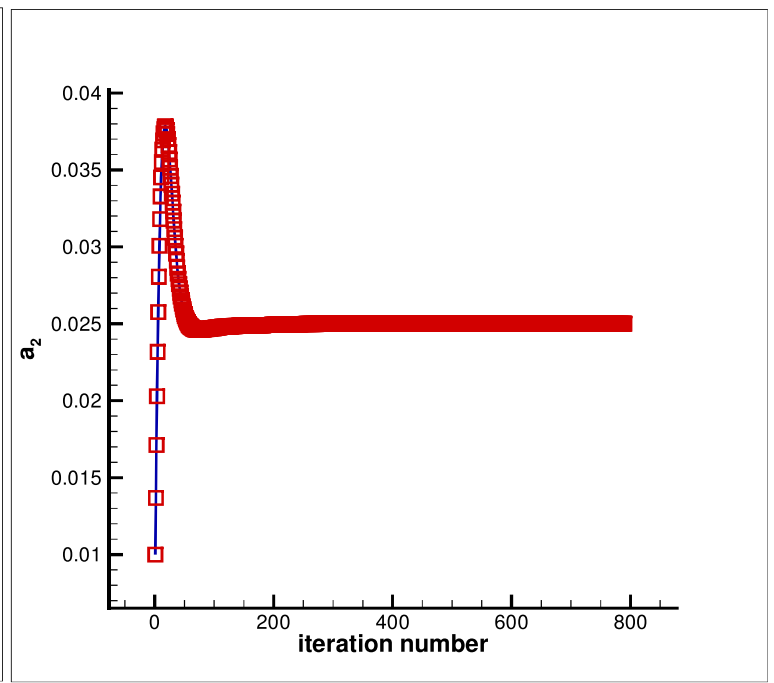

b)

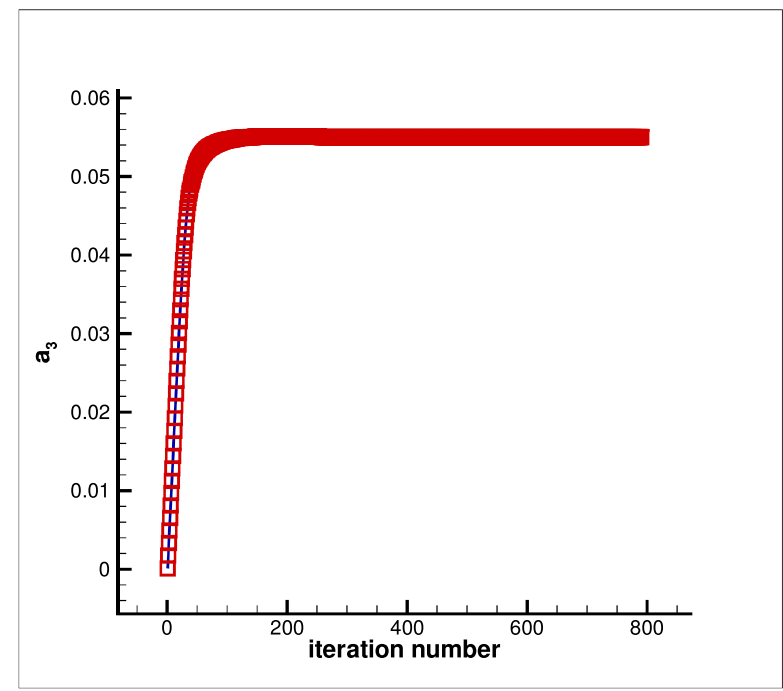

c)

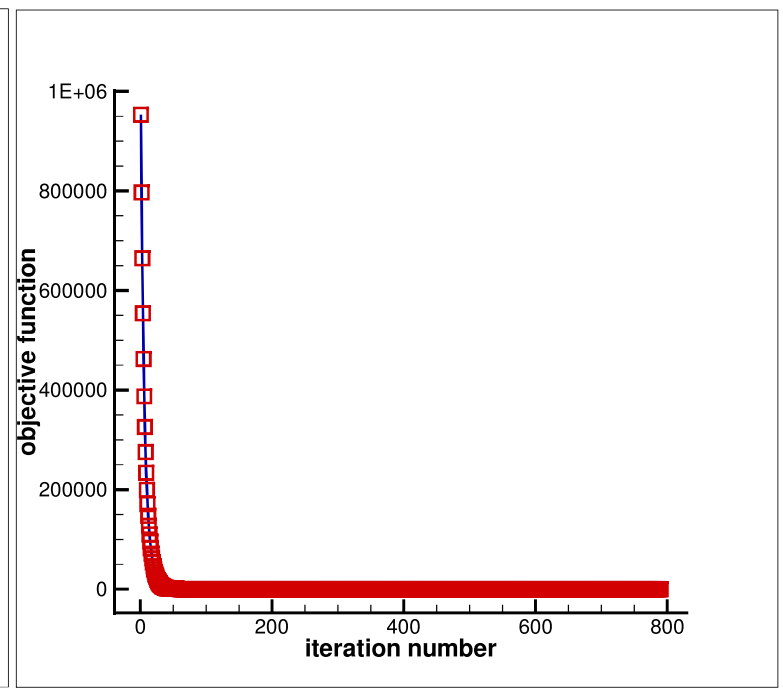

d)

Fig. 26 Estimation of $a_{1}, a_{2}, a_{3}\left(k=a_{1} e^{\left(1+a_{2} x\right)} e^{\left(1+a_{3} y\right)}\right)$ (a-c) and objective function versus iteration number (d) for initial thermal conductivity $k_{\text {initial }_{1}}=3 e^{\left(1+0.01 x_{i, j}\right)} e^{\left(1+0.0001 y_{i, j}\right)}\left(\frac{\mathrm{W}}{\mathrm{m} \cdot{ }^{\circ} \mathrm{C}}\right)$. 


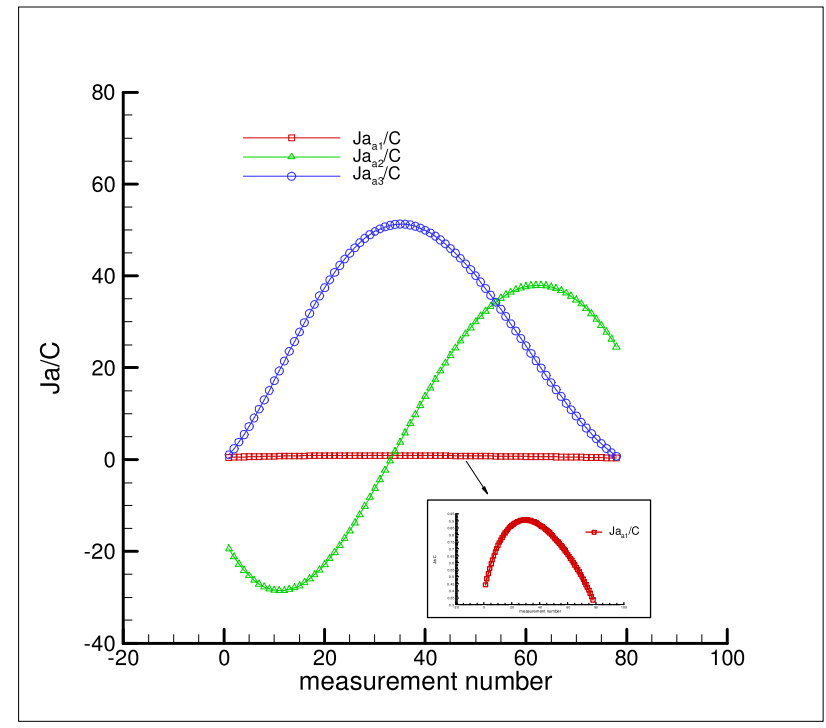

Fig. 27 Sensitivity coefficients given by Eq. (37).

Initial guess 2: $a_{1_{\text {initial }_{2}}}=25.0, \quad a_{2_{\text {initial }_{2}}}=0.00001, a_{3_{\text {initial }_{2}}}=0.00001$

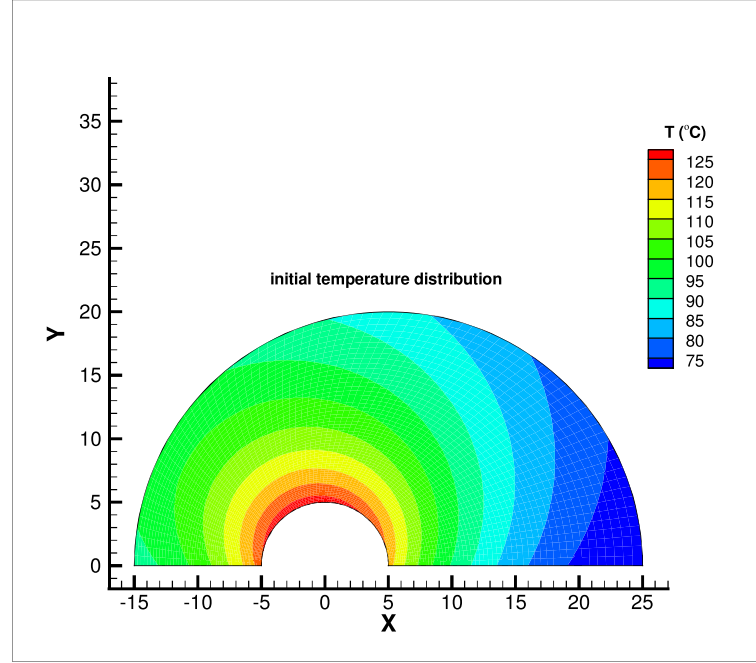

a)

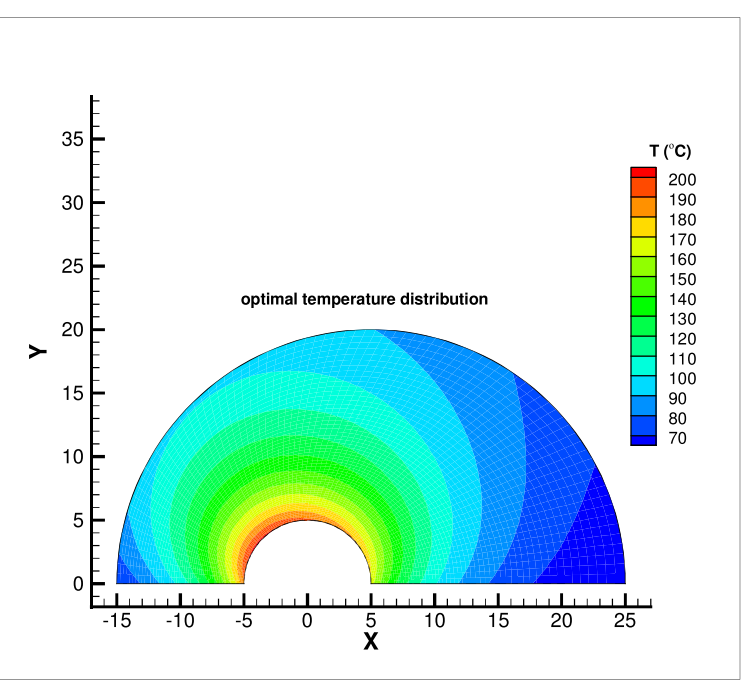

b)

Fig. 28 Initial (a) and optimal (b) temperature distribution. 


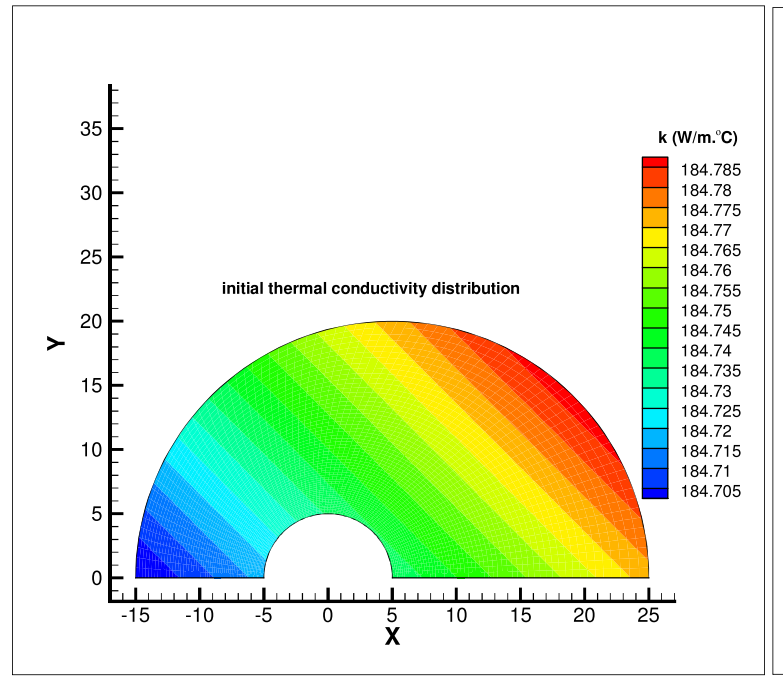

a)

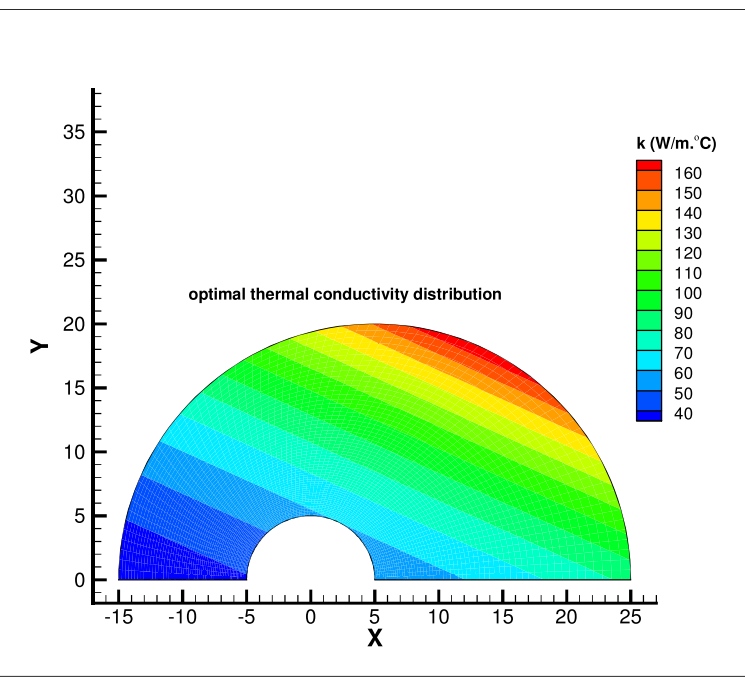

b)

Fig. 29 Initial (a) and optimal (b) thermal conductivity distribution.

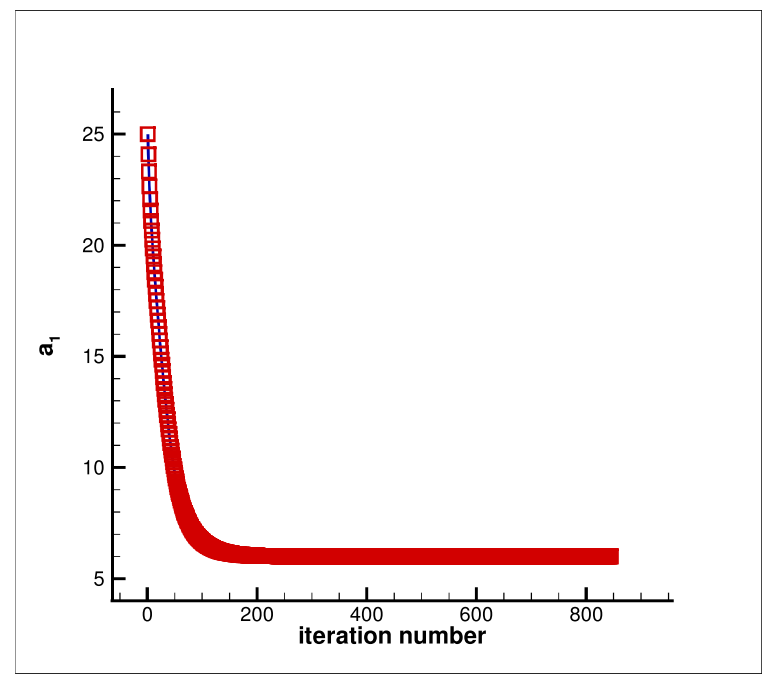

a)

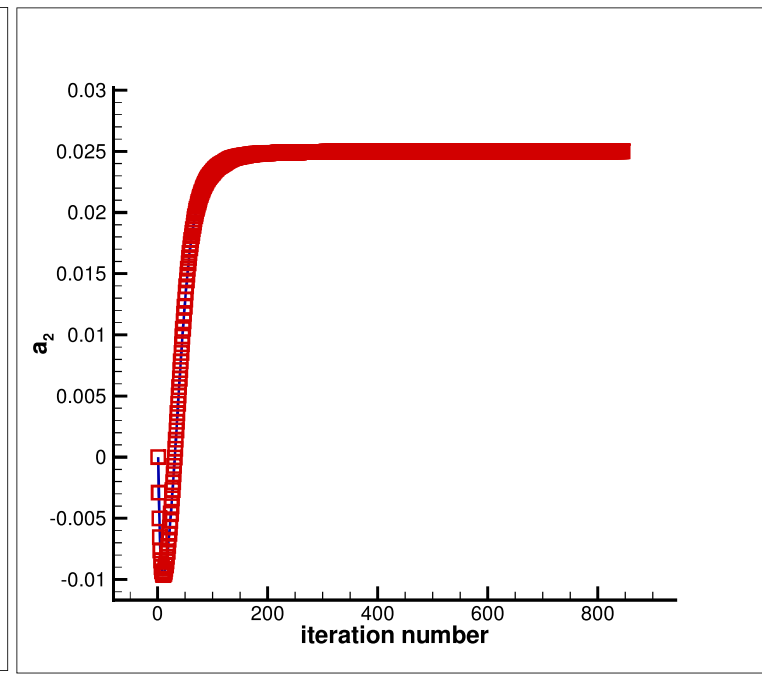

b) 


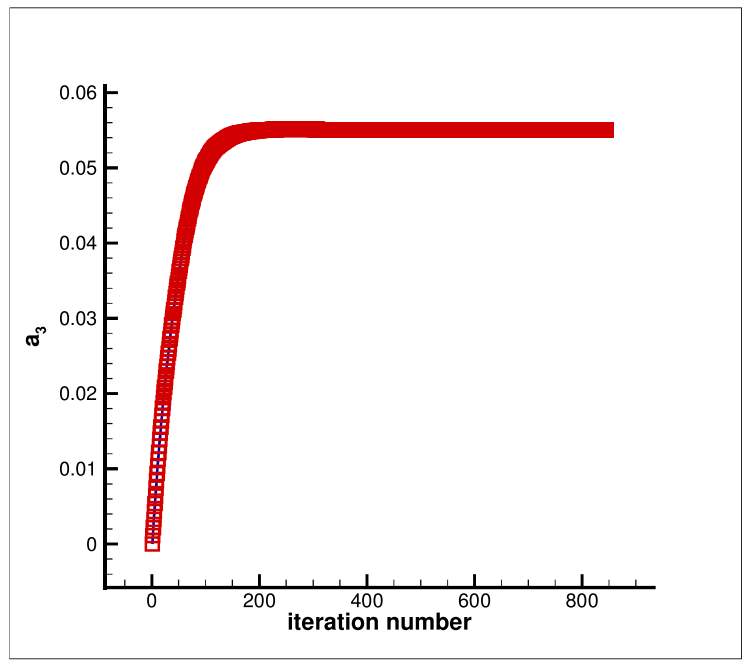

c)

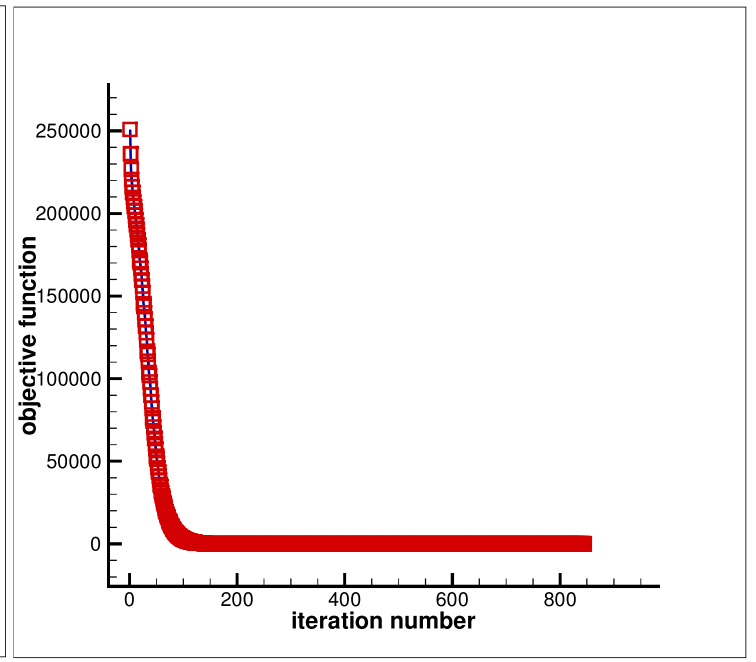

d)

Fig. 30 Estimation of $a_{1}, a_{2}, a_{3}\left(k=a_{1} e^{\left(1+a_{2} x\right)} e^{\left(1+a_{3} y\right)}\right)$ (a-c) and objective function versus iteration number (d) for initial thermal conductivity $k_{\text {initial }_{2}}=25 e^{\left(1+0.00001 x_{i, j}\right)} e^{\left(1+0.00001 y_{i, j}\right)}\left(\frac{\mathrm{W}}{\mathrm{m} \cdot{ }^{\circ} \mathrm{C}}\right)$.

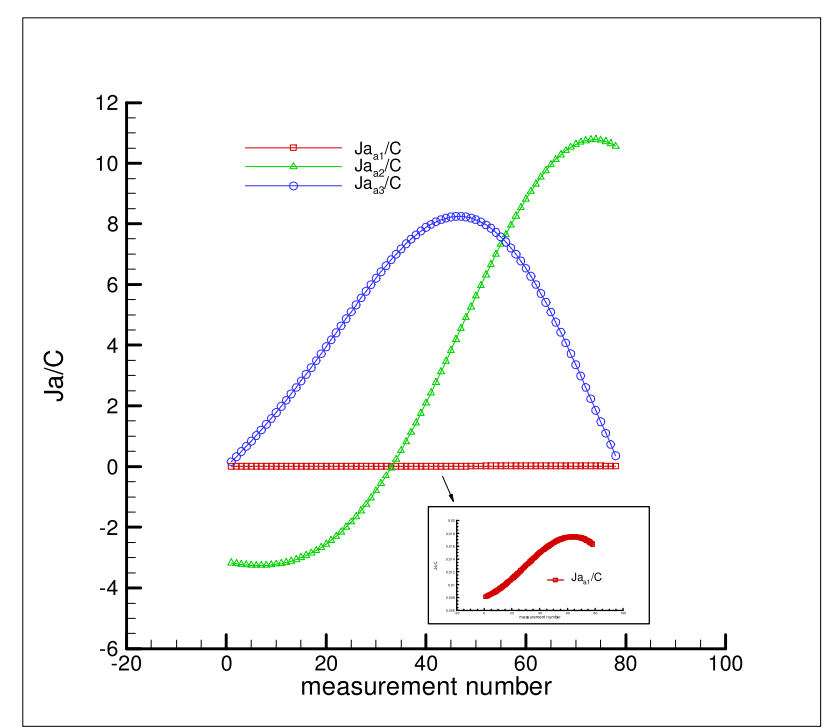

Fig. 31 Sensitivity coefficients given by Eq. (37). 
Initial 3: $a_{1_{\text {initial }_{3}}}=50.0, \quad a_{2_{\text {initial }_{3}}}=0.0, \quad a_{3_{\text {initial }_{3}}}=0.0$

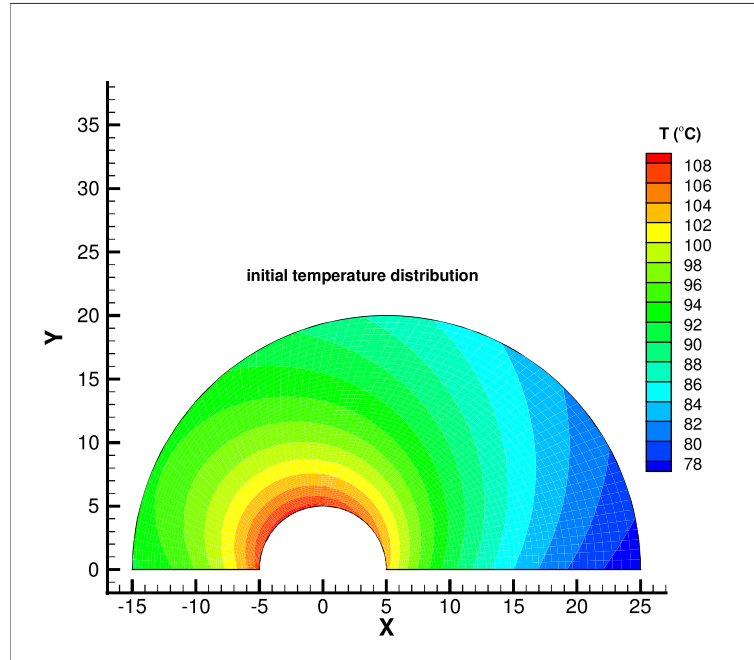

a)

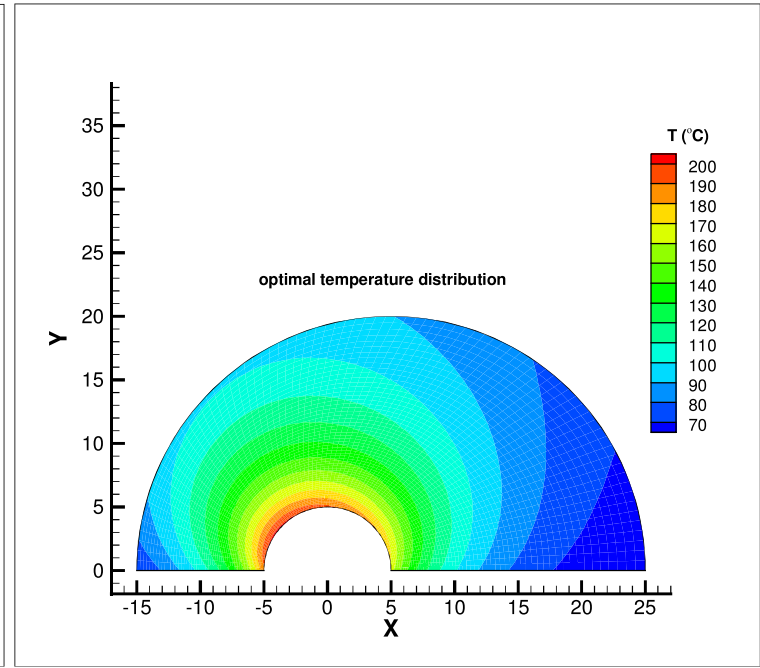

b)

Fig. 32 Initial (a) and optimal (b) temperature distribution.

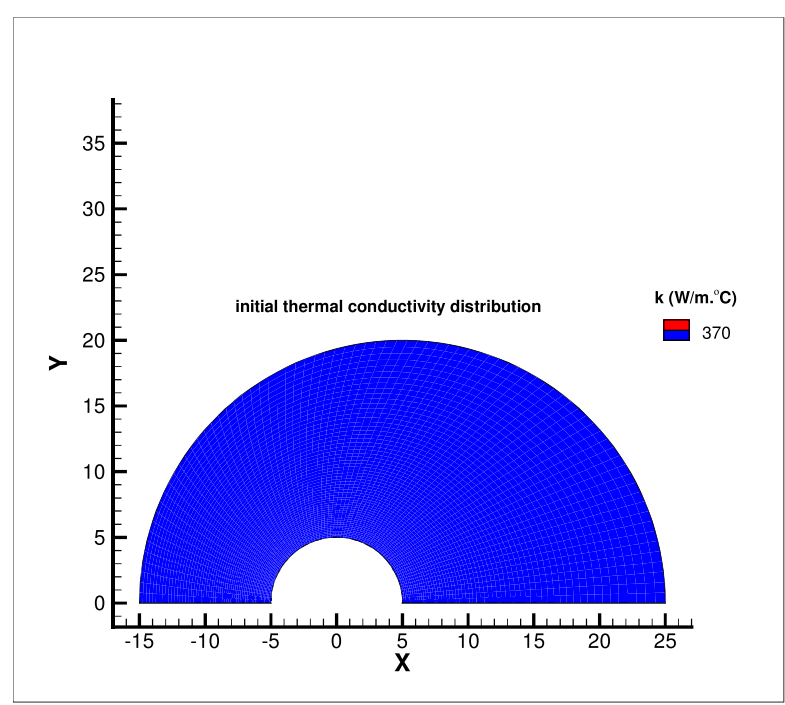

a)

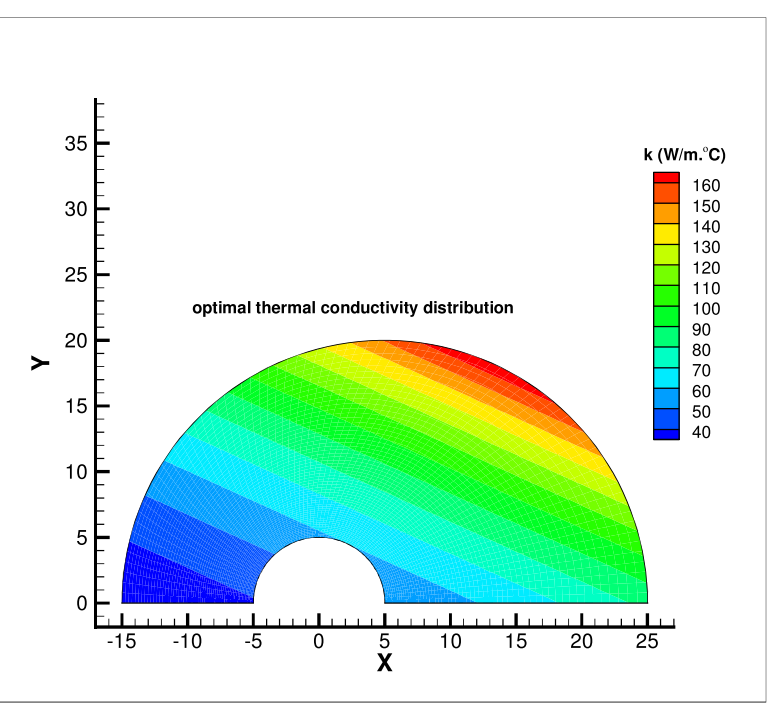

b)

Fig. 33 Initial (a) and optimal (b) thermal conductivity distribution. 


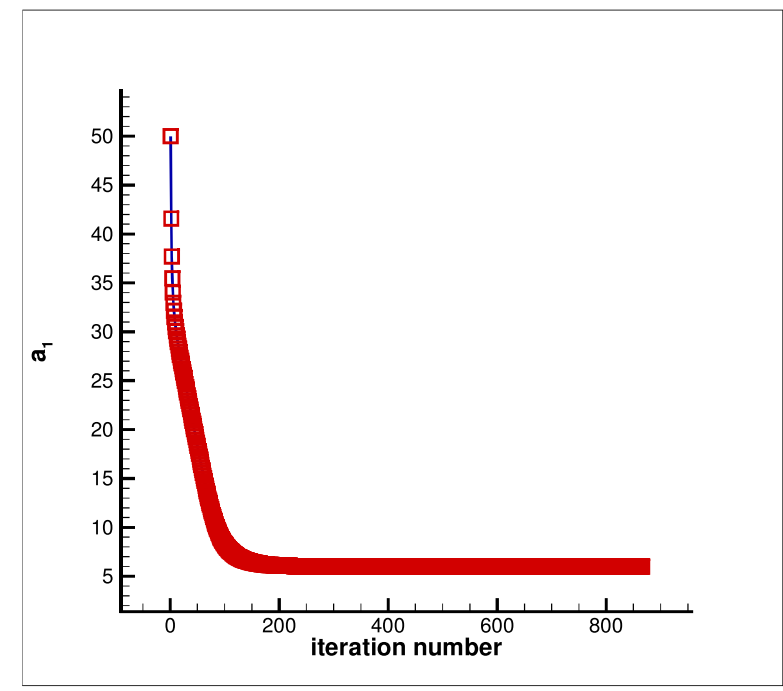

a)

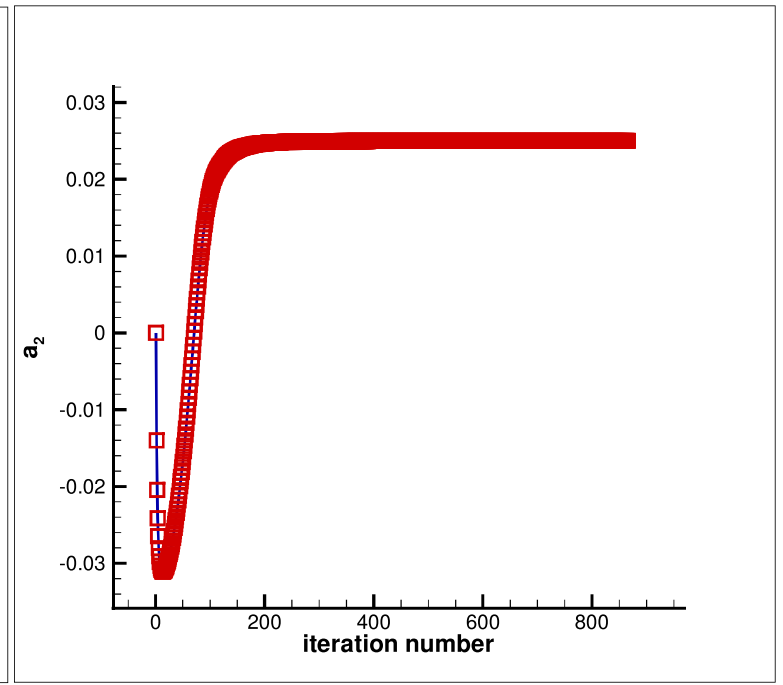

b)

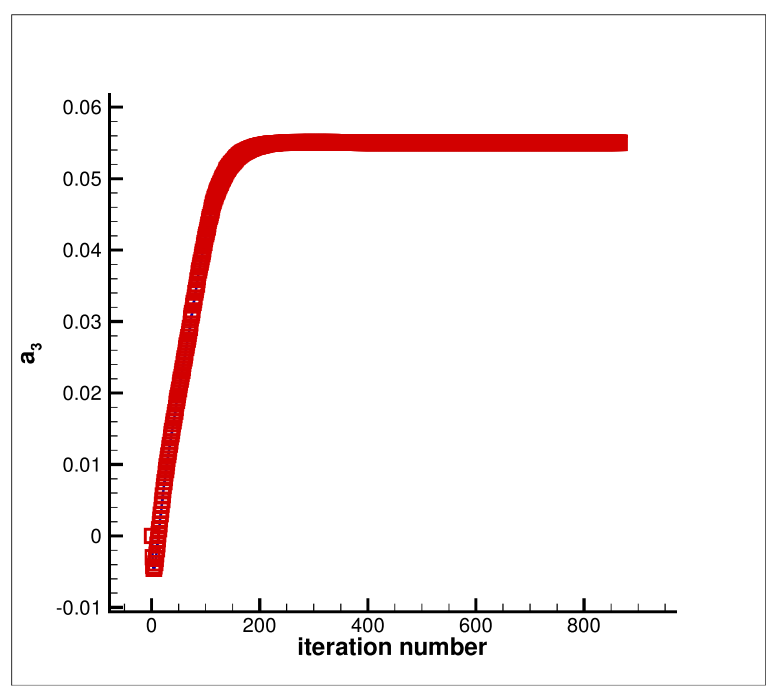

c)

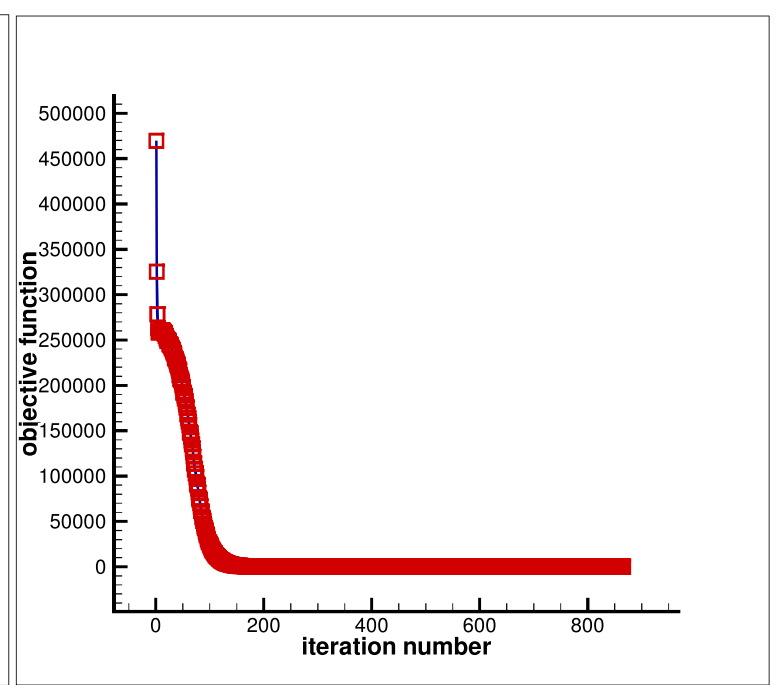

d)

Fig. 34 Estimation of $a_{1}, a_{2}, a_{3}\left(k=a_{1} e^{\left(1+a_{2} x\right)} e^{\left(1+a_{3} y\right)}\right)$ (a-c) and objective function versus iteration number (d) for initial thermal conductivity $k_{\text {initial }_{3}}=50 e^{\left(1+0.0 x_{i, j}\right)} e^{\left(1+0.0 y_{i, j}\right)}\left(\frac{\mathrm{W}}{\mathrm{m} \cdot{ }^{\circ} \mathrm{C}}\right)$. 


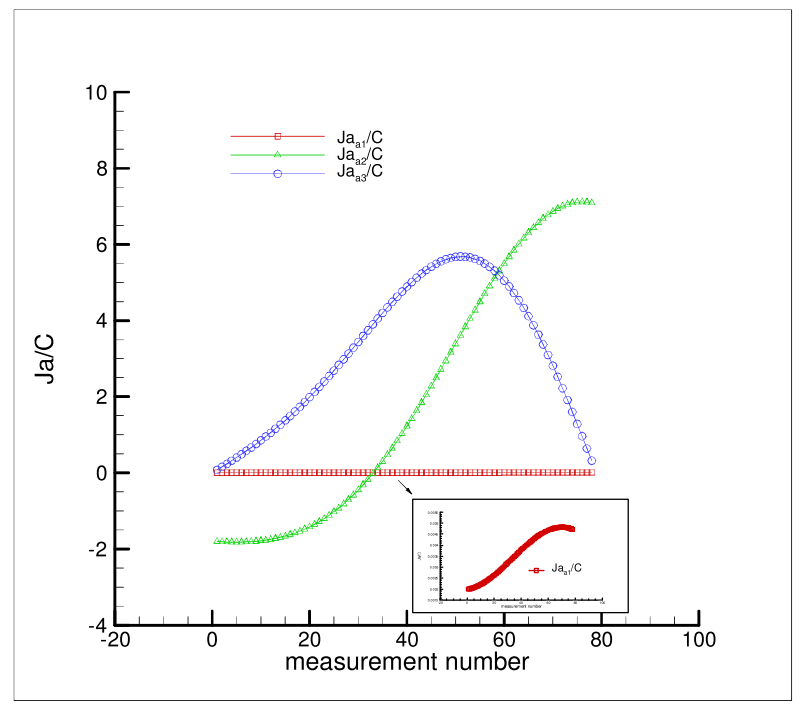

Fig. 35 Sensitivity coefficients given by Eq. (37).

Initial guess 2 (with measurement error):

$a_{1_{\text {initial }_{2}}}=25.0, \quad a_{2_{\text {initial }_{2}}}=0.00001, \quad a_{3_{\text {initial }_{2}}}=0.00001$

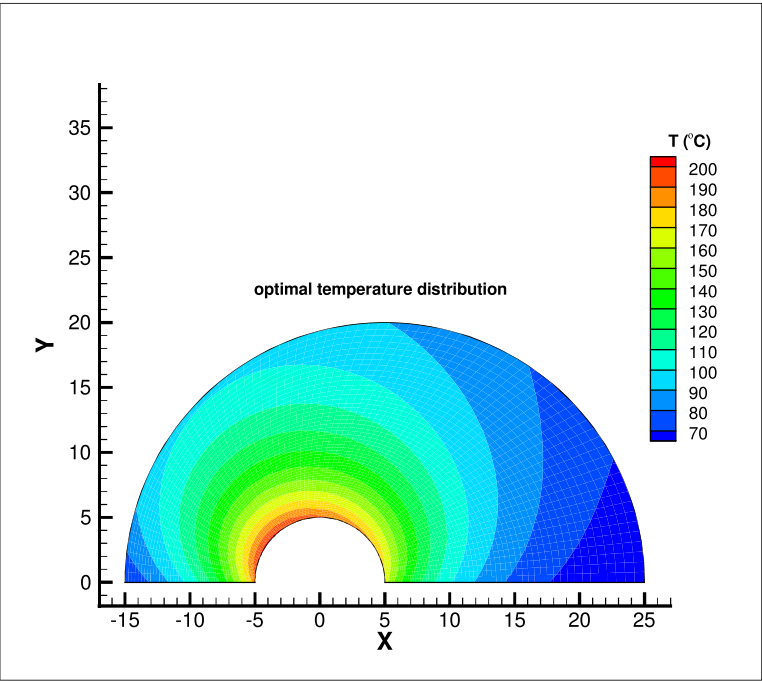

a)

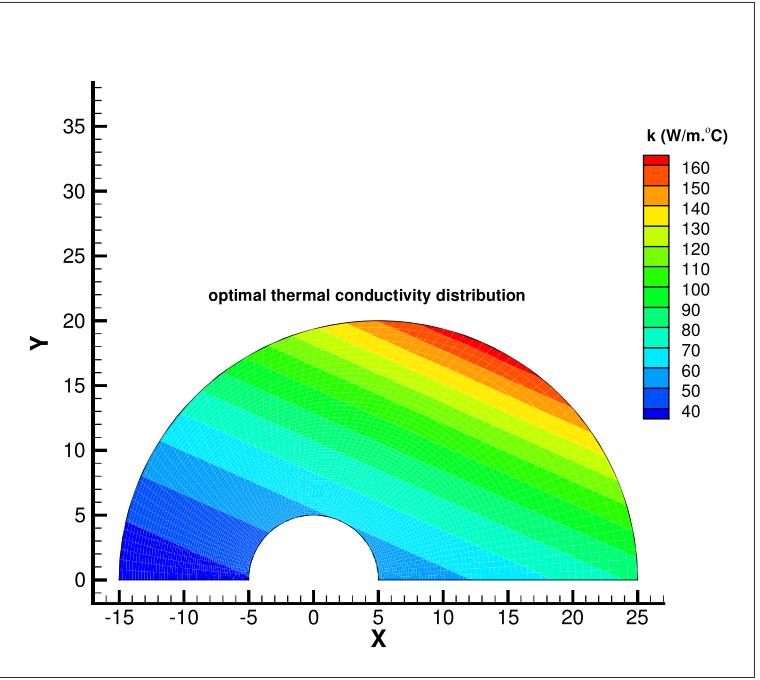

b)

Fig. 36 Optimal temperature distribution (a) and thermal conductivity (b). 


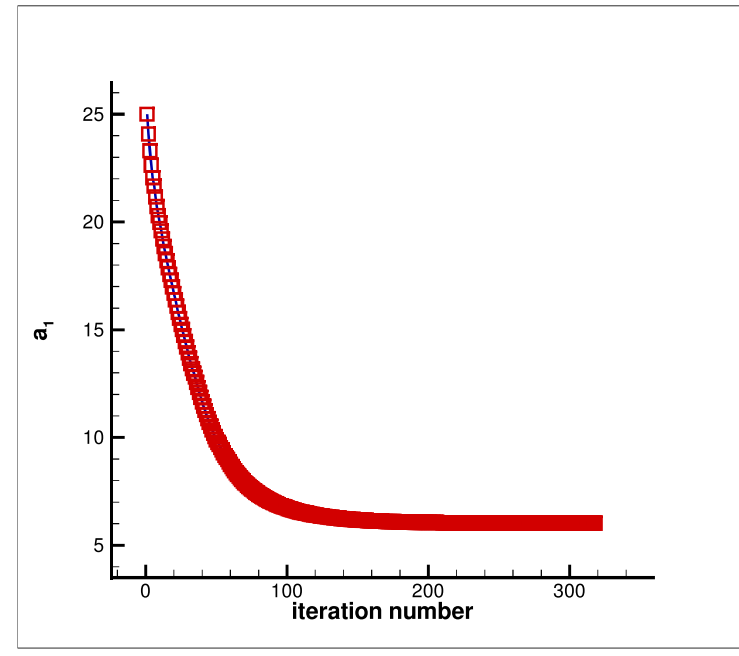

a)

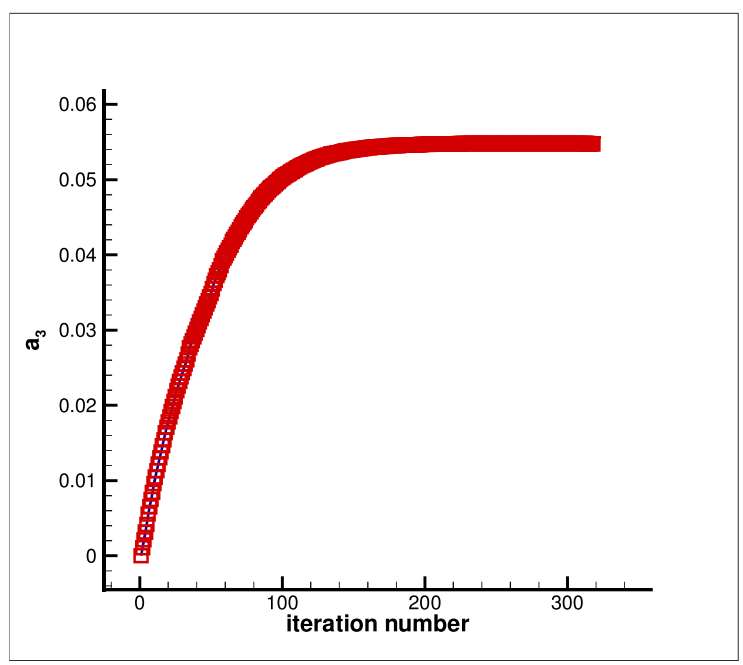

c)

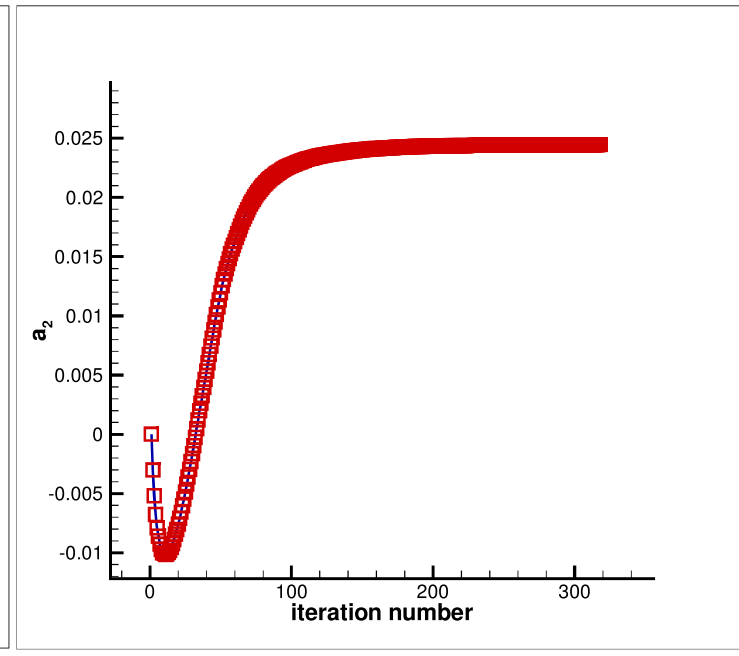

b)

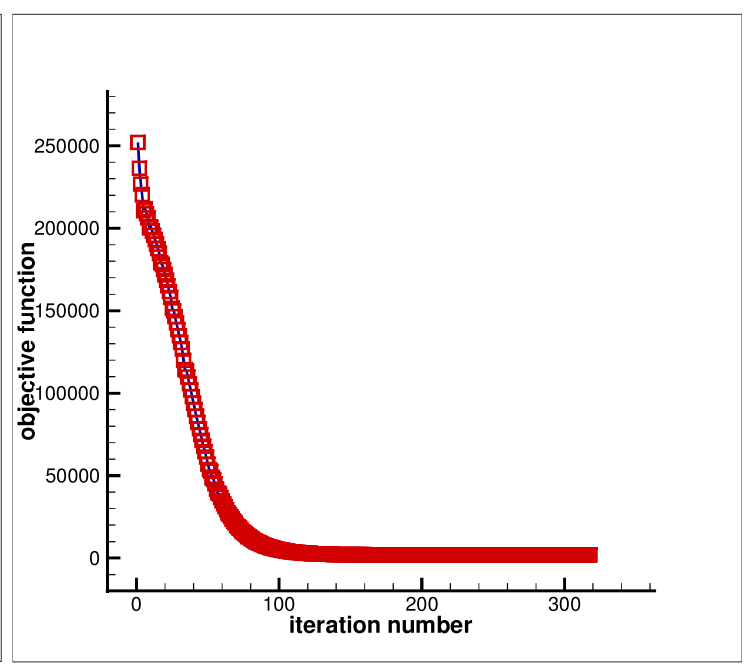

d)

Fig. 37 Estimation of $a_{1}, a_{2}, a_{3}\left(k=a_{1} e^{\left(1+a_{2} x\right)} e^{\left(1+a_{3} y\right)}\right)$ (a-c) and objective function versus iteration number (d) for initial thermal conductivity $k_{\text {initial }_{2}}=25 e^{\left(1+0.00001 x_{i, j}\right)} e^{\left(1+0.00001 y_{i, j}\right)}\left(\frac{\mathrm{W}}{\mathrm{m} \cdot{ }^{\circ} \mathrm{C}}\right)$ by considering measurement error ( $\sigma=0.5)$ 


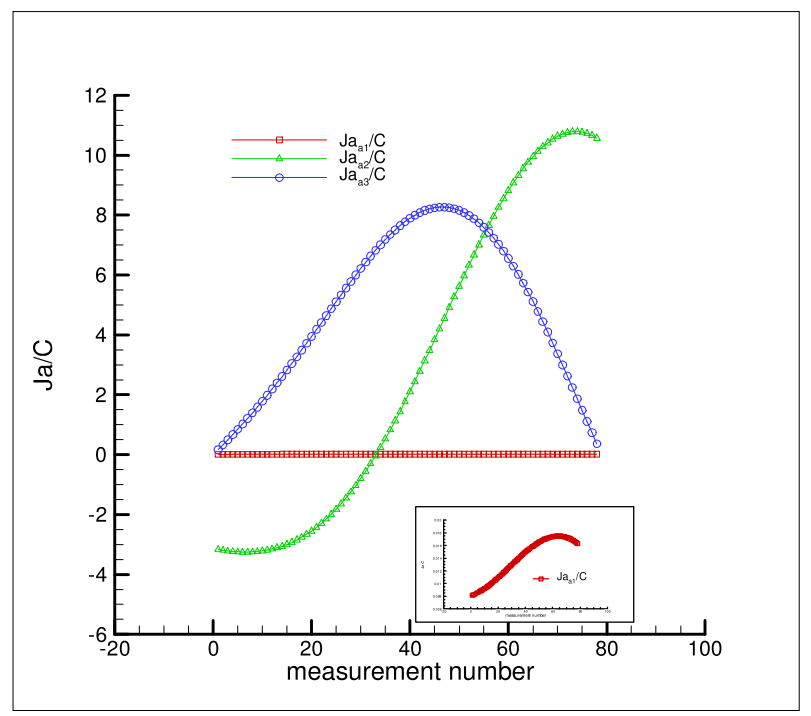

Fig. 38 Sensitivity coefficients given by Eq. (37).

In these two test cases, we used three different initial guesses to examine the accuracy, efficiency, and robustness of the inverse analysis. The initial and optimal temperature distributions obtained by application of the initial and optimal thermal conductivities are shown in Fig. 9a and Fig. 9b (Test case 1, initial guess 1), Fig. 13a and Fig. 13b, (Test case 1, initial guess 2), Fig. 17a and Fig. 17b (Test case 1, initial guess 3), Fig. 24a and Fig. 24b, (Test case 2, initial guess 1), and Fig. 28a and Fig. 28b, (Test case 2, initial guess 2), Fig. 32a and Fig. 32b (Test case 2, initial guess 3), respectively. The initial and optimal thermal conductivity distributions are depicted in Fig. 10a and Fig. 10b (Test case 1, initial guess 1), Fig. 14a and Fig. 14b (Test case 1, initial guess 2), Fig. 18a and Fig. 18b (Test case 1, initial guess 3), Fig. 25a and Fig. 25b (Test case 2, initial guess 1), and Fig. 29a and Fig. 29b (Test case 2, initial guess 2), Fig. 33a and Fig. 33b (Test case 2, initial guess 3), respectively. As can be seen, the distributions of temperature and retrieved thermal conductivity in both test cases with different initial guesses are in excellent agreement with the desired ones. In Test cases 1 and 2 with initial guess 1 , because the parameter $a_{3}$ is smaller than the parameter $a_{2}$, $a_{2_{\text {initial }_{1}}}=0.05$ and $a_{3_{\text {initial }_{1}}}=0.001$ for Test case 1 and $a_{2_{\text {initial }_{1}}}=0.01$ and $a_{3_{\text {initial }_{1}}}=0.0001$ for Test case 2 , the variation of the thermal conductivity in the $y-$ direction is smaller than the variation of the thermal conductivity in the $x$ - direction 
(Fig. 10a and Fig. 25a). In Test case 1 with initial guess 2, however, the parameter $a_{2}$ is smaller than the parameter $a_{3}, a_{2_{\text {initial }_{2}}}=0.0001, a_{3_{\text {initial }_{2}}}=0.1$, hence the variation of the thermal conductivity in the $x$ - direction is negligible compared to the variation of the thermal conductivity in the $y$ - direction (Fig. 14a). As shown in Fig. 11, Fig. 15, and Fig. 19 for Test case 1 and Fig. 26, Fig. 30, and Fig. 34 for Test case 2, a 100\% reduction in the objective function and complete recovering of the values for $a_{1}, a_{2}$, and $a_{3}$ (the variable thermal conductivity components) are achieved by starting the optimization process from three different initial guesses which are numerically far from the desired ones thereby demonstrating the robustness of the proposed numerical approach. In view of the large number of iterations needed to recover the unknown variables, the computation time reveals that the proposed method is very efficient. The details of the results, including the initial and final values for $a_{1}, a_{2}$, and $a_{3}$, the initial and final values of the objective function, the computation time, the number of iterations, and the percentage of the decrease in the objective function, are given in Table 3 (for both cases of no measurement error and measurement error). In case of the measurement error $(\sigma=0.5)$, there is also an approximately $100 \%$ reduction in the objective function. As shown in Table 3 as well as Fig. 22 (for Test case 1) and Fig. 37 (for Test case 2), the error in recovering the parameter $a_{l}, l=1,2,3$ (constant component of the variable thermal conductivity) is negligible and the distributions of temperature and retrieved thermal conductivity in the presence of the measurement error are also in very good agreement with the desired ones (Fig. 21 for Test case 1 and Fig. 36 for Test case 2). Moreover, the effect of the mesh size on the inverse analysis is explored using two more mesh sizes of $20 \times 20$ and $130 \times 130$ for Test case 1 using the third initial guess. As shown in Fig. 39 to Fig. 43 (the initial temperature and thermal conductivity distributions are not shown), the results are not affected by the mesh size and the desired parameters are completely recovered. The sensitivity coefficients for all test cases with all initial guesses (with and without the measurement error) are shown in Fig. 12, Fig. 16, Fig. 20, Fig. 23, Fig. 27, Fig. 31, Fig. 35, Fig. 38, Fig. 41, and Fig. 44. It can be seen that, in all cases, the sensitivity coefficients with respect to the parameters $a_{1}, a_{2}$, and $a_{3}$ are linearly independent (as expected; see Eq. (64)) and therefore the estimation of these parameters are feasible. The results from a statistical 
analysis for the estimated parameters including the standard deviation of estimated parameters and $99 \%$ confidence interval for each estimated parameter are given in Table 4. The results show that quite accurate estimates are obtained even for the measurement error of $\sigma=0.5$.

The results are obtained by a FORTRAN compiler and computations are run on a PC with Intel Core 15 and 6G RAM. A tolerance of $10^{-7}$ is used in iterative loops to increase the accuracy of results.

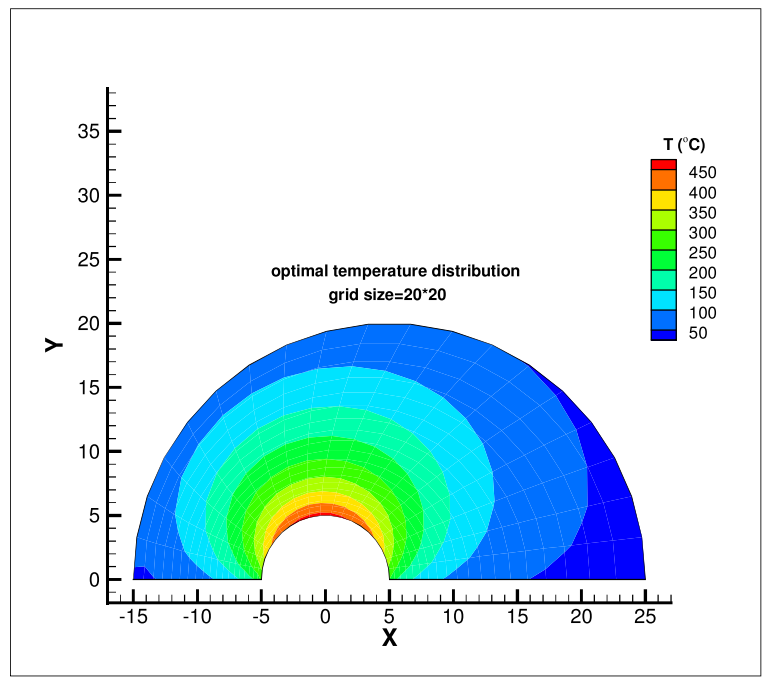

a)

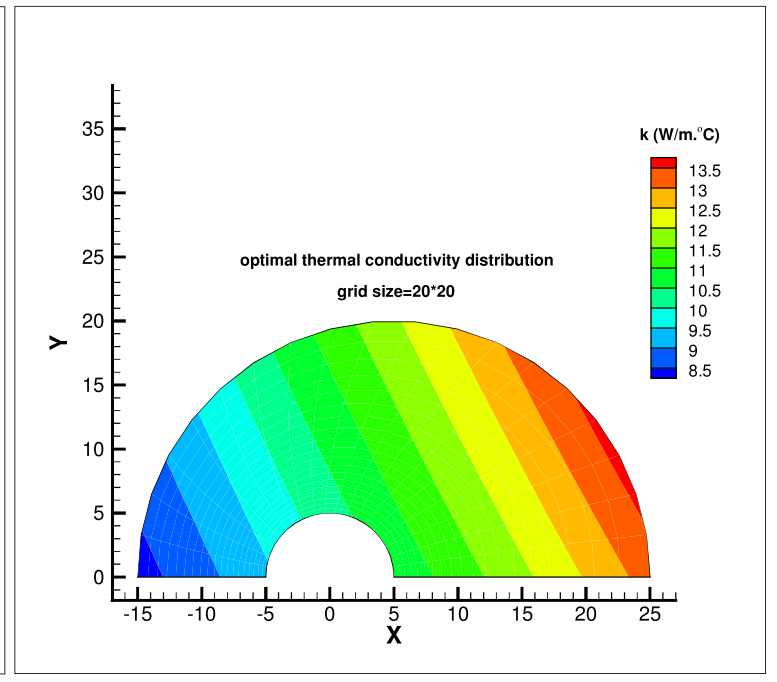

b)

Fig. 39 Optimal temperature distribution (a) and thermal conductivity (b) using a grid size of $20 \times 20$. The third initial guess in Test case $1, k_{\text {initial }_{3}}=300\left(1+0.0 x_{i, j}\right)^{2}\left(1+0.0 y_{i, j}\right)^{2}\left(\frac{\mathrm{W}}{\mathrm{m} \cdot{ }^{\circ} \mathrm{C}}\right)$, is used. 


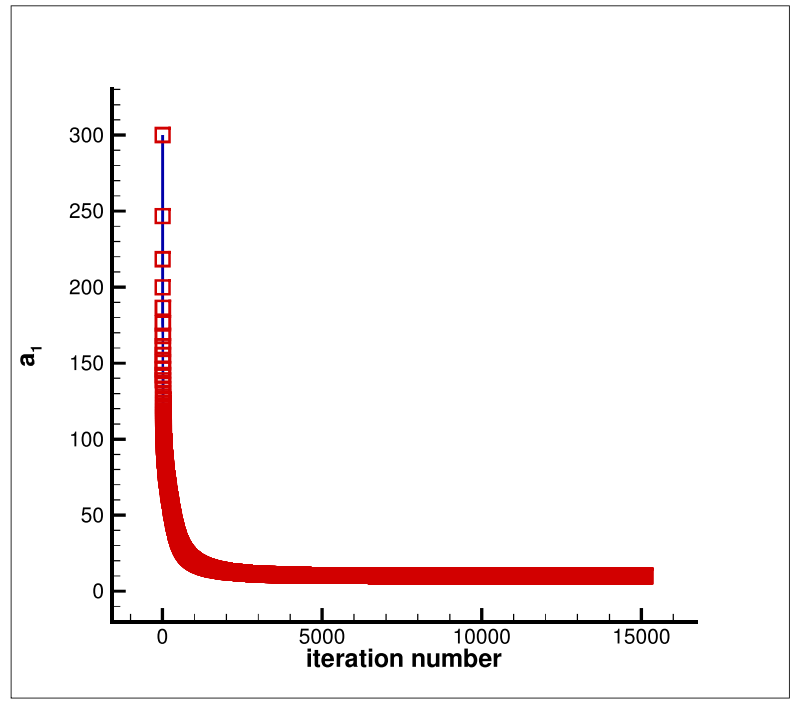

a)

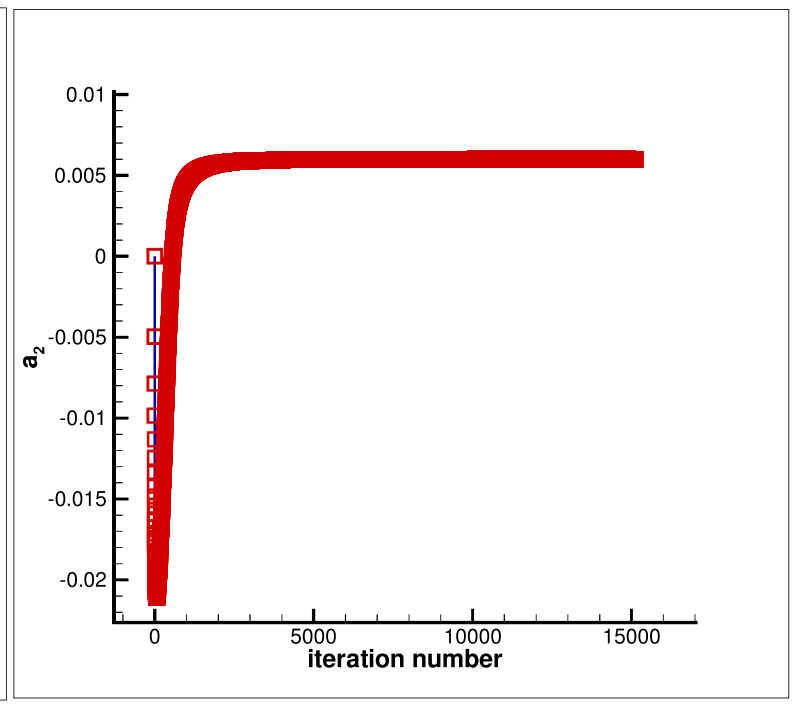

b)

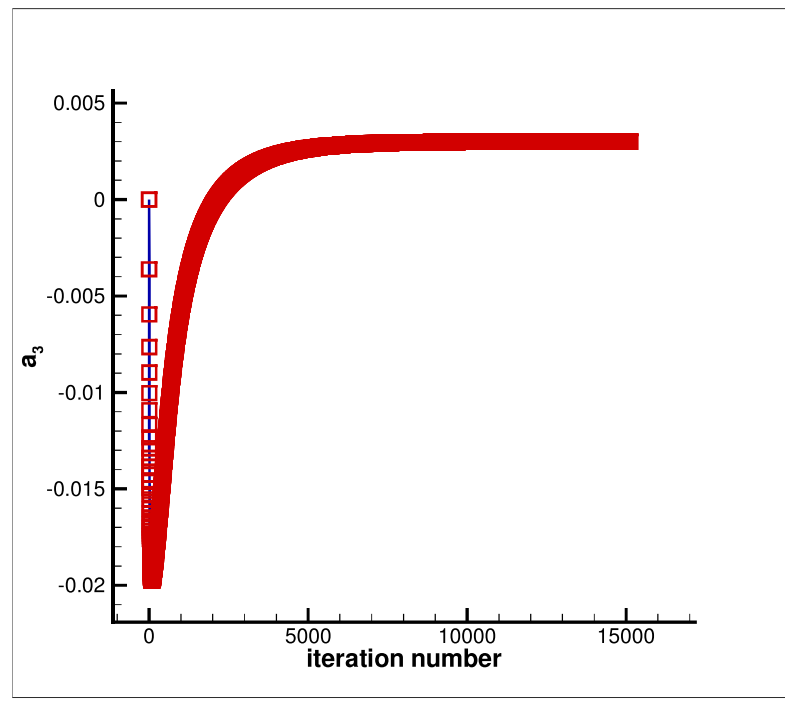

c)

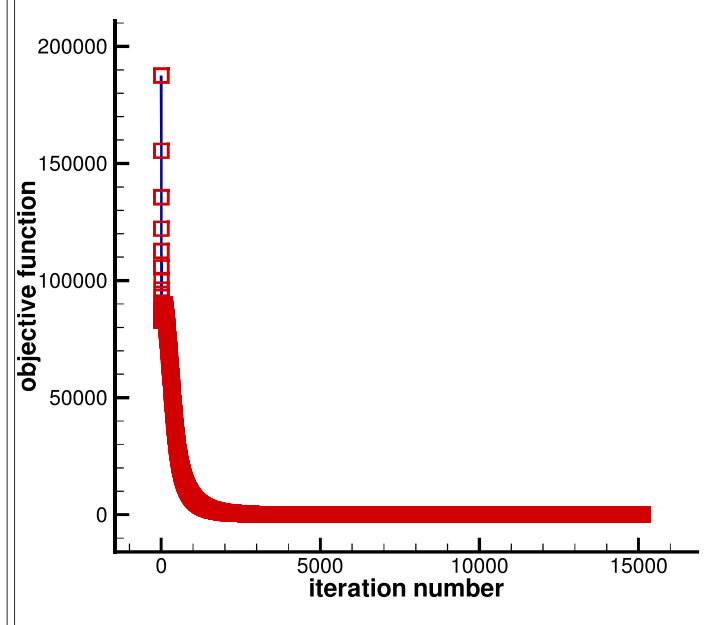

d)

Fig. 40 Estimation of $a_{1}, a_{2}, a_{3}\left(k=a_{1}\left(1+a_{2} x\right)^{2}\left(1+a_{3} y\right)^{2}\right)$, (Test case 1), using a grid size of $20 \times 20$ (a-c) and objective function versus iteration number $(d)$ for initial thermal conductivity

$$
k_{\text {initial }_{3}}=300\left(1+0.0 x_{i, j}\right)^{2}\left(1+0.0 y_{i, j}\right)^{2}\left(\frac{\mathrm{W}}{\mathrm{m} .{ }^{\circ} \mathrm{C}}\right) \text {. }
$$




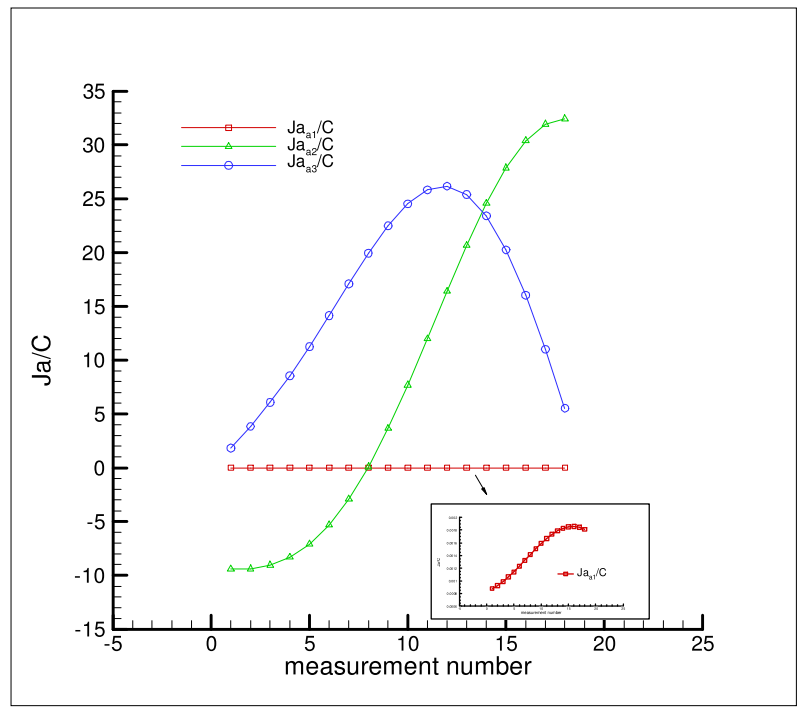

Fig. 41 Sensitivity coefficients given by Eq. (37).

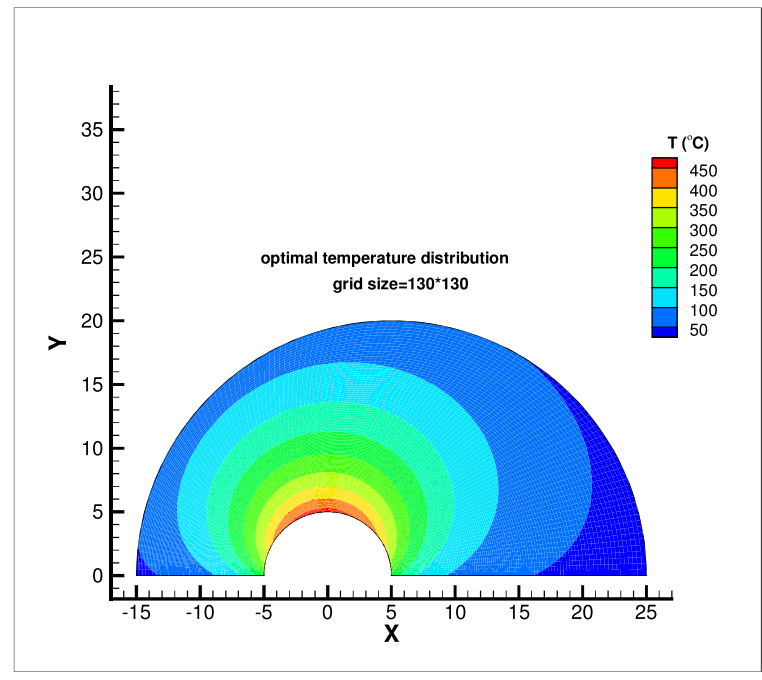

a)

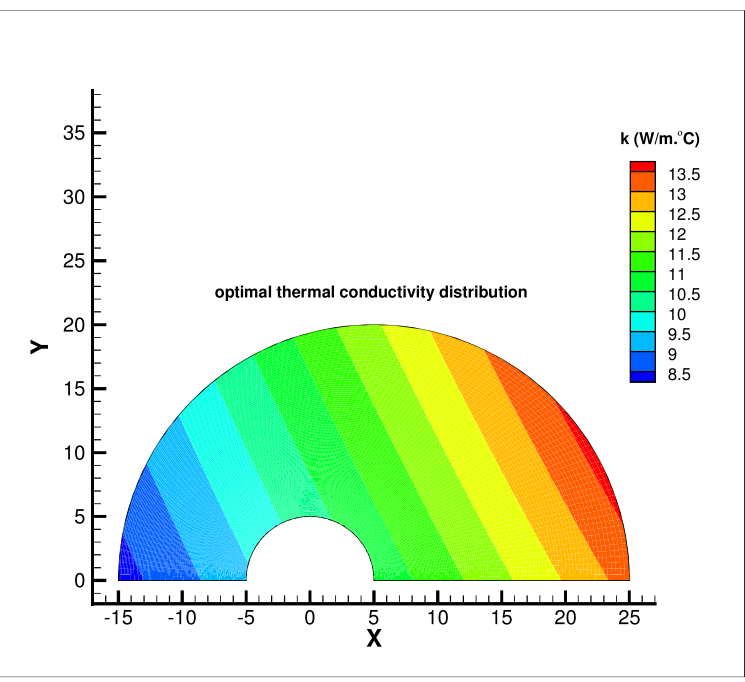

b)

Fig. 42 Optimal temperature distribution (a) and thermal conductivity (b) using a grid size of $130 \times 130$. The third initial guess in Test case $1, k_{\text {initial }_{3}}=300\left(1+0.0 x_{i, j}\right)^{2}\left(1+0.0 y_{i, j}\right)^{2}\left(\frac{\mathrm{W}}{\mathrm{m} \cdot{ }^{\circ} \mathrm{C}}\right)$, is used. 


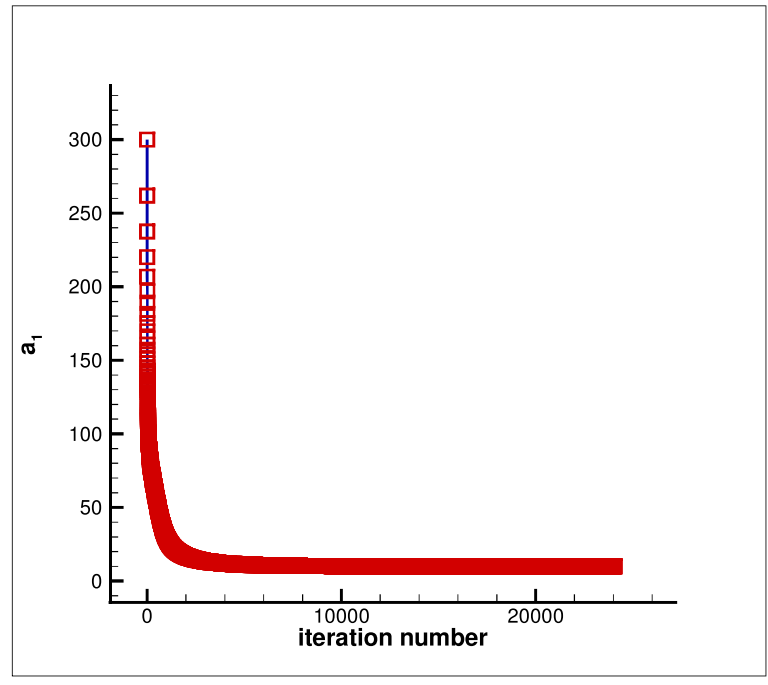

a)

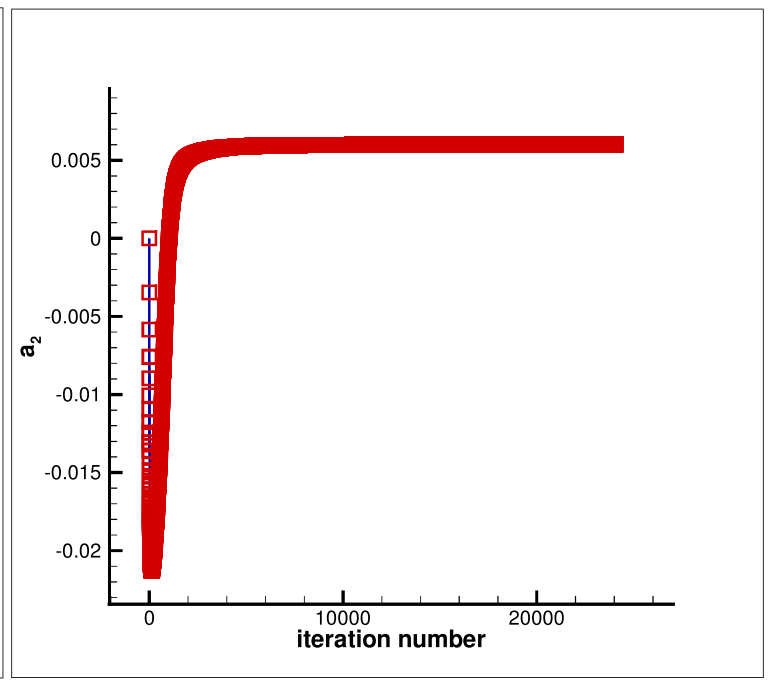

b)

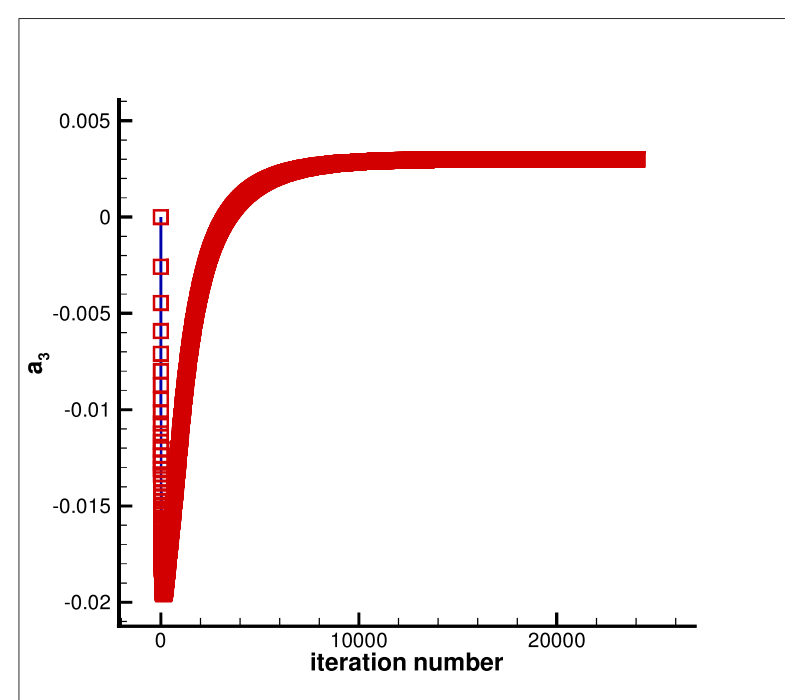

c)

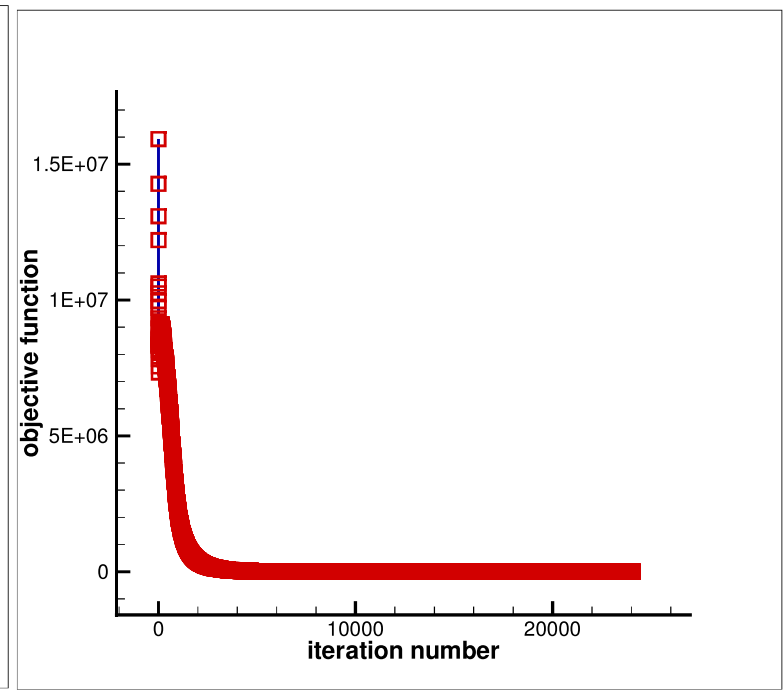

d)

Fig. 43 Estimation of $a_{1}, a_{2}, a_{3}\left(k=a_{1}\left(1+a_{2} x\right)^{2}\left(1+a_{3} y\right)^{2}\right)$, (Test case 1$)$, using a grid size of $130 \times 130$ (a-c) and objective function versus iteration number $(d)$ for initial thermal conductivity

$$
k_{\text {initial }_{3}}=300\left(1+0.0 x_{i, j}\right)^{2}\left(1+0.0 y_{i, j}\right)^{2}\left(\frac{\mathrm{W}}{\mathrm{m} \cdot{ }^{\circ} \mathrm{C}}\right) .
$$




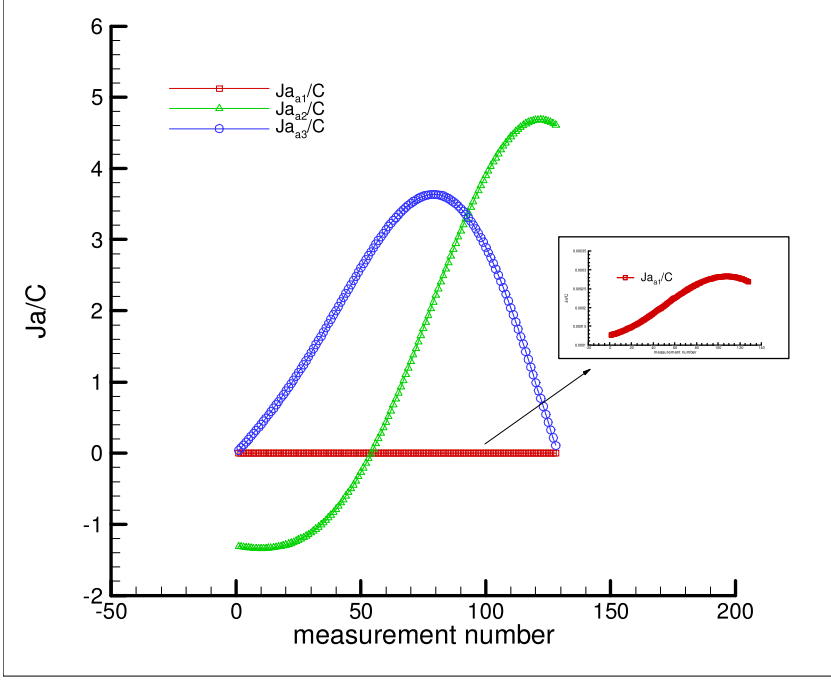

Fig. 44 Sensitivity coefficients given by Eq. (37) 


\begin{tabular}{|c|c|c|c|c|c|c|c|}
\hline $\begin{array}{c}\text { Test case } \\
\& \\
\text { Grid size }\end{array}$ & $\begin{array}{l}\text { Desired } \\
\text { value }\end{array}$ & $\begin{array}{l}\text { Initial } \\
\text { (guess) } \\
\text { value }\end{array}$ & $\begin{array}{l}\text { Final } \\
\text { value }\end{array}$ & $\begin{array}{l}\text { Temperature } \\
\text { measurement } \\
\text { error }\end{array}$ & $\begin{array}{c}\text { Initial value } \\
\text { of } \mathcal{J}\end{array}$ & $\begin{array}{c}\text { Minimum } \\
\text { value of } \mathcal{J}\end{array}$ & $\begin{array}{c}\text { Reduction } \\
\text { in objective } \\
\text { function \& } \\
\text { computation time }\end{array}$ \\
\hline $\begin{array}{c}1 \\
(\text { initial 1) } \\
80 \times 60\end{array}$ & $\begin{array}{l}a_{1}=10.0 \\
a_{2}=0.006 \\
a_{3}=0.003\end{array}$ & $\begin{array}{l}a_{1}=25.0 \\
a_{2}=0.05 \\
a_{3}=0.001\end{array}$ & $\begin{array}{l}a_{1}=10.0 \\
a_{2}=0.006 \\
a_{3}=0.003\end{array}$ & $\sigma=0$ & $\begin{array}{l}102430.66 \\
(C=10)\end{array}$ & $\begin{array}{l}9.995 \times 10^{-6} \\
(C=10)\end{array}$ & $\begin{array}{l}100 \% \\
(11 \mathrm{~min}: 22 \mathrm{~s}) \\
\text { (543 iterations) }\end{array}$ \\
\hline $\begin{array}{c}1 \\
(\text { initial 2) } \\
80 \times 60\end{array}$ & $\begin{array}{l}a_{1}=10.0 \\
a_{2}=0.006 \\
a_{3}=0.003\end{array}$ & $\begin{array}{l}a_{1}=2.0 \\
a_{2}=0.0001 \\
a_{3}=0.1\end{array}$ & $\begin{array}{l}a_{1}=10.0 \\
a_{2}=0.006 \\
a_{3}=0.003\end{array}$ & $\sigma=0$ & $\begin{array}{l}60454.21 \\
(C=10)\end{array}$ & $\begin{array}{l}9.99 \times 10^{-6} \\
(C=10)\end{array}$ & $\begin{array}{l}100 \% \\
(26 \mathrm{~min}: 46 \mathrm{~s}) \\
\text { (2267 iterations) }\end{array}$ \\
\hline $\begin{array}{c}1 \\
(\text { initial 3) } \\
80 \times 60\end{array}$ & $\begin{array}{l}a_{1}=10.0 \\
a_{2}=0.006 \\
a_{3}=0.003\end{array}$ & $\begin{array}{l}a_{1}=300.0 \\
a_{2}=0.0 \\
a_{3}=0.0\end{array}$ & $\begin{array}{l}a_{1}=10.0 \\
a_{2}=0.006 \\
a_{3}=0.003\end{array}$ & $\sigma=0$ & $\begin{array}{l}953478.76 \\
(C=100)\end{array}$ & $\begin{array}{l}9.98 \times 10^{-6} \\
(C=100)\end{array}$ & $\begin{array}{l}100 \% \\
(53 \mathrm{~min}: 44 \mathrm{~s}) \\
\text { (5472 iterations) }\end{array}$ \\
\hline $\begin{array}{c}1 \\
(\text { initial 3) } \\
20 \times 20\end{array}$ & $\begin{array}{l}a_{1}=10.0 \\
a_{2}=0.006 \\
a_{3}=0.003\end{array}$ & $\begin{array}{l}a_{1}=300.0 \\
a_{2}=0.0 \\
a_{3}=0.0\end{array}$ & $\begin{array}{l}a_{1}=10.0 \\
a_{2}=0.006 \\
a_{3}=0.003\end{array}$ & $\sigma=0$ & $\begin{array}{l}187533.93 \\
(C=100)\end{array}$ & $\begin{array}{l}9.99 \times 10^{-6} \\
(C=100)\end{array}$ & $\begin{array}{l}100 \% \\
(4 \mathrm{~min}) \\
(15127 \text { iterations })\end{array}$ \\
\hline $\begin{array}{c}1 \\
(\text { initial 3) } \\
130 \times 130\end{array}$ & $\begin{array}{l}a_{1}=10.0 \\
a_{2}=0.006 \\
a_{3}=0.003\end{array}$ & $\begin{array}{l}a_{1}=300.0 \\
a_{2}=0.0 \\
a_{3}=0.0\end{array}$ & $\begin{array}{l}a_{1}=10.0 \\
a_{2}=0.006 \\
a_{3}=0.003\end{array}$ & $\sigma=0$ & $\begin{array}{l}15926988.92 \\
(C=1000)\end{array}$ & $\begin{array}{l}9.99 \times 10^{-4} \\
(C=1000)\end{array}$ & $\begin{array}{l}100 \% \\
(280 \mathrm{~min}) \\
(24066 \text { iterations })\end{array}$ \\
\hline $\begin{array}{c}1 \\
(\text { initial 1) } \\
80 \times 60\end{array}$ & $\begin{array}{l}a_{1}=10.0 \\
a_{2}=0.006 \\
a_{3}=0.003\end{array}$ & $\begin{array}{l}a_{1}=25.0 \\
a_{2}=0.05 \\
a_{3}=0.001\end{array}$ & $\begin{array}{l}a_{1}=10.093 \\
(\text { error }=0.9 \%) \\
a_{2}=0.0056 \\
(\text { error }=6.7 \%) \\
a_{3}=0.0028 \\
(\text { error }=6.7 \%)\end{array}$ & $\sigma=0.5$ & $\begin{array}{l}104294.26 \\
(C=10)\end{array}$ & $\begin{array}{l}185.999 \\
(C=10)\end{array}$ & $\begin{array}{l}\sim 100 \% \\
(327 \text { iterations })\end{array}$ \\
\hline $\begin{array}{c}2 \\
(\text { initial 1) } \\
80 \times 60\end{array}$ & $\begin{array}{l}a_{1}=6.0 \\
a_{2}=0.025 \\
a_{3}=0.055\end{array}$ & $\begin{array}{l}a_{1}=3.0 \\
a_{2}=0.01 \\
a_{3}=0.0001\end{array}$ & $\begin{array}{l}a_{1}=6.0 \\
a_{2}=0.025 \\
a_{3}=0.055\end{array}$ & $\sigma=0$ & $\begin{array}{l}953060.39 \\
(C=100)\end{array}$ & $\begin{array}{l}9.999 \times 10^{-5} \\
(C=100)\end{array}$ & $\begin{array}{l}100 \% \\
(31 \mathrm{~min}: 11 \mathrm{~s}) \\
\text { (789 iterations) }\end{array}$ \\
\hline $\begin{array}{c}2 \\
(\text { initial 2) } \\
80 \times 60\end{array}$ & $\begin{array}{l}a_{1}=6.0 \\
a_{2}=0.025 \\
a_{3}=0.055\end{array}$ & $\begin{array}{l}a_{1}=25.0 \\
a_{2}=0.00001 \\
a_{3}=0.00001\end{array}$ & $\begin{array}{l}a_{1}=6.0 \\
a_{2}=0.025 \\
a_{3}=0.055\end{array}$ & $\sigma=0$ & $\begin{array}{l}250817.05 \\
(C=100)\end{array}$ & $\begin{array}{l}9.996 \times 10^{-5} \\
(C=100)\end{array}$ & $\begin{array}{l}100 \% \\
(73 \mathrm{~min}: 43 \mathrm{~s}) \\
\text { (844 iterations) }\end{array}$ \\
\hline $\begin{array}{c}2 \\
(\text { initial 3) } \\
80 \times 60\end{array}$ & $\begin{array}{l}a_{1}=6.0 \\
a_{2}=0.025 \\
a_{3}=0.055\end{array}$ & $\begin{array}{l}a_{1}=50.0 \\
a_{2}=0.0 \\
a_{3}=0.0\end{array}$ & $\begin{array}{l}a_{1}=6.0 \\
a_{2}=0.025 \\
a_{3}=0.055\end{array}$ & $\sigma=0$ & $\begin{array}{l}469652.50 \\
(C=100)\end{array}$ & $\begin{array}{l}9.999 \times 10^{-5} \\
(C=100)\end{array}$ & $\begin{array}{l}100 \% \\
(107 \mathrm{~min}: 55 \mathrm{~s}) \\
\text { (865 iterations) }\end{array}$ \\
\hline $\begin{array}{c}2 \\
(\text { initial 2) } \\
80 \times 60\end{array}$ & $\begin{array}{l}a_{1}=6.0 \\
a_{2}=0.025 \\
a_{3}=0.055\end{array}$ & $\begin{array}{l}a_{1}=25.0 \\
a_{2}=0.00001 \\
a_{3}=0.00001\end{array}$ & $\begin{array}{l}a_{1}=6.02 \\
(\text { error }=0.3 \%) \\
a_{2}=0.0245 \\
(\text { error }=2.0 \%) \\
a_{3}=0.0548 \\
(\text { error }=0.36 \%)\end{array}$ & $\sigma=0.5$ & $\begin{array}{l}252072.28 \\
(C=100)\end{array}$ & $\begin{array}{l}1866.98 \\
(C=100)\end{array}$ & $\begin{array}{l}\sim 100 \% \\
(317 \text { iterations })\end{array}$ \\
\hline
\end{tabular}

Table 3 A summary of results for the estimation of the spatially varying thermal conductivity components $a_{1}, a_{2}$, and $a_{3}$. 


\begin{tabular}{|c|c|c|c|c|c|c|}
\hline $\begin{array}{l}\text { Test case } \\
\qquad \& \\
\text { grid size }\end{array}$ & $\begin{array}{c}\text { Desired } \\
\text { value for } \\
\text { parameter }\end{array}$ & $\begin{array}{l}\text { Initial } \\
\text { (guess) } \\
\text { value for } \\
\text { parameter }\end{array}$ & $\begin{array}{c}\text { Final } \\
\text { value for } \\
\text { parameter }\end{array}$ & $\begin{array}{c}\text { Standard } \\
\text { deviation of } \\
\text { temperature } \\
\text { measurement } \\
\text { errors }\end{array}$ & $\begin{array}{c}\text { Standard } \\
\text { deviation of } \\
\text { estimated } \\
\text { parameter, } \\
\text { (Eq. (77)) } \\
\end{array}$ & $\begin{array}{l}\text { Confidence Interval } \\
\text { (Eq. (73)) }\end{array}$ \\
\hline $\begin{array}{c}1 \\
(\text { initial 1) } \\
80 \times 60\end{array}$ & $\begin{array}{l}a_{1}=10.0 \\
a_{2}=0.006 \\
a_{3}=0.003\end{array}$ & $\begin{array}{l}a_{1}=25.0 \\
a_{2}=0.05 \\
a_{3}=0.001\end{array}$ & $\begin{array}{l}a_{1}=10.093 \\
(\text { error }=0.9 \%) \\
a_{2}=0.0056 \\
(\text { error }=6.7 \%) \\
a_{3}=0.0028 \\
(\text { error }=6.7 \%)\end{array}$ & $\sigma=0.5$ & $\begin{aligned} \sigma_{a_{1}} & =0.2229 \\
\sigma_{a_{2}} & =0.0010 \\
\sigma_{a_{3}} & =0.0007\end{aligned}$ & $\begin{array}{l}9.519 \leq a_{1} \leq 10.667 \\
0.0029 \leq a_{2} \leq 0.0082 \\
0.0009 \leq a_{3} \leq 0.0047\end{array}$ \\
\hline $\begin{array}{c}2 \\
(\text { initial 2) } \\
80 \times 60\end{array}$ & $\begin{array}{l}a_{1}=6.0 \\
a_{2}=0.025 \\
a_{3}=0.055\end{array}$ & $\begin{array}{l}a_{1}=25.0 \\
a_{2}=0.00001 \\
a_{3}=0.00001\end{array}$ & $\begin{array}{l}a_{1}=6.02 \\
(\text { error }=0.3 \%) \\
a_{2}=0.0245 \\
(\text { error }=2.0 \%) \\
a_{3}=0.0548 \\
(\text { error }=0.36 \%)\end{array}$ & $\sigma=0.5$ & $\begin{aligned} \sigma_{a_{1}} & =0.5503 \\
\sigma_{a_{2}} & =0.0060 \\
\sigma_{a_{3}} & =0.0073\end{aligned}$ & $\begin{array}{l}4.60 \leq a_{1} \leq 7.44 \\
0.0090 \leq a_{2} \leq 0.0400 \\
0.0359 \leq a_{3} \leq 0.0737\end{array}$ \\
\hline
\end{tabular}

Table 4 Statistical analysis results for the estimation of parameters $a_{1}, a_{2}$, and $a_{3}$ in the presence of measurement error.

\section{Other boundary surfaces as measurement place}

In this study, the boundary surface $\Gamma_{2}$ (Fig. 1) is chosen as the place where the simulated measurements are performed. In this section, the effect of considering other boundary surfaces such as $\Gamma_{1}$ (the surface of the applied heat flux $\dot{q}$ ) on the accuracy of the estimated parameters will be investigated. Considering the boundary surface $\Gamma_{1}$, we can write the objective function as

$$
\mathcal{J}=C \sum_{i=2}^{M-1}\left(T_{i, 1}-T_{(i, 1)_{m}}\right)^{2}
$$

The derivative of the objective function with respect to the unknown variables can be obtained as

$$
\frac{\partial \mathcal{J}}{\partial a_{l}}=2 C \sum_{i=2}^{M-1}\left(T_{i, 1}-T_{(i, 1)_{m}}\right) \frac{\partial T_{i, 1}}{\partial a_{l}}=2 \sum_{i=2}^{M-1}\left(T_{i, 1}-T_{(i, 1)_{m}}\right) C \frac{\partial T_{i, 1}}{\partial a_{l}}
$$


where $l=1,2,3$. The sensitivity coefficients $C \frac{\partial T_{i, 1}}{\partial a_{l}}(l=1,2,3)$ can be derived by considering the boundary condition relating to the boundary surface $\Gamma_{1}$. The associated boundary condition can be written as

$$
\begin{gathered}
\dot{q}=k \frac{\partial T}{\partial n_{1}} \\
\dot{q}=k\left(\frac{-1}{J \sqrt{\gamma}}\left(\gamma T_{\eta}-\beta T_{\xi}\right)\right)
\end{gathered}
$$

at the surface $\Gamma_{1}$, we have

$$
\begin{gathered}
T_{\xi}=\frac{1}{2}\left(T_{i+1,1}-T_{i-1,1}\right) \\
T_{\eta}=\frac{1}{2}\left(-3 T_{i, 1}+4 T_{i, 2}-T_{i, 3}\right)
\end{gathered}
$$

Therefore, Eq. (82) becomes

$$
\dot{q}=k_{i, 1}\left[\frac{-1}{J \sqrt{\gamma}}\left(\gamma \frac{-3 T_{i, 1}+4 T_{i, 2}-T_{i, 3}}{2}-\beta \frac{T_{i+1,1}-T_{i-1,1}}{2}\right)\right]
$$

where the coefficients $J, \gamma$, and $\beta$ defined in Eq. (13) are computed using finitedifference coefficients associated with the surface $\Gamma_{1}$. Solving Eq. (85) for $T_{i, 1}$ gives the temperature distribution on the boundary surface $\Gamma_{1}$ as follows

$$
T_{i, 1}=\frac{\frac{2}{3} \dot{q} J}{k_{i, 1} \sqrt{\gamma}}+\frac{1}{3}\left(\frac{4 \gamma T_{i, 2}-\gamma T_{i, 3}-\beta T_{i+1,1}+\beta T_{i-1,1}}{\gamma}\right)
$$

By considering the exponential material gradation (quadratic material gradation case can be treated in a similar fashion), $k=a_{1} e^{\left(1+a_{2} x\right)} e^{\left(1+a_{3} y\right)}$, Eq. (86) is written as 


$$
T_{i, 1}=\frac{\frac{2}{3} \dot{q} J}{a_{1} e^{\left(1+a_{2} x_{i, 1}\right)} e^{\left(1+a_{3} y_{i, 1}\right)} \sqrt{\gamma}}+\frac{1}{3}\left(\frac{4 \gamma T_{i, 2}-\gamma T_{i, 3}-\beta T_{i+1,1}+\beta T_{i-1,1}}{\gamma}\right)
$$

Thus the sensitivity coefficients $C \frac{\partial T_{i, 1}}{\partial a_{l}}(l=1,2,3)$ can be explicitly expressed by differentiating the obtained expression for $T_{i, 1}$ with respect to $a_{l}, l=1,2,3$, as follows

$$
\begin{gathered}
\frac{\partial T_{i, 1}}{\partial a_{1}}=-\frac{\frac{2}{3} \dot{q} J e^{\left(-2-a_{2} x_{i, 1}-a_{3} y_{i, 1}\right)}}{\sqrt{\gamma} a_{1}^{2}} \\
\frac{\partial T_{i, 1}}{\partial a_{2}}=-\frac{\frac{2}{3} x_{i, 1} \dot{q} J e^{\left(-2-a_{2} x_{i, 1}-a_{3} y_{i, 1}\right)}}{\sqrt{\gamma} a_{1}} \\
\frac{\partial T_{i, 1}}{\partial a_{3}}=-\frac{\frac{2}{3} y_{i, 1} \dot{q} J e^{\left(-2-a_{2} x_{i, 1}-a_{3} y_{i, 1}\right)}}{\sqrt{\gamma} a_{1}}
\end{gathered}
$$

As can be seen in the above equations, the parameter $a_{1}$ should not be zero. In order to evaluate the accuracy of the above sensitivity coefficients, Test case 2 with initial 2 (for both cases of no measurement error and the measurement error of $\sigma=0.5$ ) is repeated. The results are given in Table 5 and the statistical error analysis is given in Table 6 . As can be seen, the unknown parameters are again recovered accurately representing the accuracy of the sensitivity coefficients at the boundary surface $\Gamma_{1}$ even in the presence of a measurement error of $\sigma=0.5$. 


\begin{tabular}{|c|c|c|c|c|c|c|c|}
\hline $\begin{array}{l}\text { Test case } \\
\qquad \& \\
\text { Grid size }\end{array}$ & $\begin{array}{l}\text { Desired } \\
\text { value }\end{array}$ & $\begin{array}{l}\text { Initial } \\
\text { (guess) } \\
\text { value }\end{array}$ & $\begin{array}{l}\text { Final } \\
\text { value }\end{array}$ & $\begin{array}{c}\text { Tempcrature } \\
\text { measurement } \\
\text { error }\end{array}$ & $\begin{array}{c}\text { Initial value } \\
\text { of } \mathcal{J}\end{array}$ & $\begin{array}{l}\text { Minimum } \\
\text { value of } \mathfrak{g}\end{array}$ & $\begin{array}{c}\text { Reduction } \\
\text { in objective } \\
\text { function \& } \\
\text { computation } \\
\text { time }\end{array}$ \\
\hline $\begin{array}{c}2 \\
\text { (initial 2) } \\
80 \times 60\end{array}$ & $\begin{array}{l}a_{1}=6.0 \\
a_{2}=0.025 \\
a_{3}=0.055\end{array}$ & $\begin{array}{l}a_{1}=25.0 \\
a_{2}=0.00001 \\
a_{3}=0.00001\end{array}$ & $\begin{array}{l}a_{1}=6.0 \\
a_{2}=0.025 \\
a_{3}=0.055\end{array}$ & $\sigma=0$ & $\begin{array}{l}331904235.4 \\
(C=1000)\end{array}$ & $\begin{array}{l}9.979 \times 10^{-5} \\
(C=1000)\end{array}$ & $\begin{array}{l}100 \% \\
\text { (4722 iterations) }\end{array}$ \\
\hline $\begin{array}{c}2 \\
(\text { initial 2) } \\
80 \times 60\end{array}$ & $\begin{array}{l}a_{1}=6.0 \\
a_{2}=0.025 \\
a_{3}=0.055\end{array}$ & $\begin{array}{l}a_{1}=25.0 \\
a_{2}=0.00001 \\
a_{3}=0.00001\end{array}$ & $\begin{array}{l}a_{1}=6.027 \\
(\text { error }=0.45 \%) \\
a_{2}=0.0253 \\
(\text { error }=1.2 \%) \\
a_{3}=0.0542 \\
(\text { error }=1.45 \%)\end{array}$ & $\sigma=0.5$ & $\begin{array}{l}332033244.7 \\
(C=1000)\end{array}$ & $\begin{array}{l}19496.44 \\
(C=1000)\end{array}$ & $\begin{array}{l}\sim 100 \% \\
(1900 \text { iterations })\end{array}$ \\
\hline
\end{tabular}

Table 5 A summary of results for the estimation of the spatially varying thermal conductivity components $a_{1}, a_{2}$, and $a_{3}$ by considering the boundary surface $\Gamma_{1}$ as the temperature measurement place.

\begin{tabular}{|c|c|c|c|c|c|c|}
\hline $\begin{array}{l}\text { Test case } \\
\qquad \& \\
\text { grid size }\end{array}$ & $\begin{array}{c}\text { Desired } \\
\text { value for } \\
\text { parameter }\end{array}$ & $\begin{array}{c}\text { Initial } \\
\text { (guess) } \\
\text { value for } \\
\text { parameter }\end{array}$ & $\begin{array}{c}\text { Final } \\
\text { value for } \\
\text { parameter }\end{array}$ & $\begin{array}{c}\text { Standard } \\
\text { deviation of } \\
\text { temperature } \\
\text { measurement } \\
\text { errors }\end{array}$ & $\begin{array}{c}\text { Standard } \\
\text { deviation of } \\
\text { estimated } \\
\text { parameter, } \\
\text { (Eq. (77)) }\end{array}$ & $\begin{array}{c}\text { Confidence Interval } \\
(\text { Eq. }(73))\end{array}$ \\
\hline $\begin{array}{c}2 \\
(\text { initial 2) } \\
80 \times 60\end{array}$ & $\begin{array}{l}a_{1}=6.0 \\
a_{2}=0.025 \\
a_{3}=0.055\end{array}$ & $\begin{array}{l}a_{1}=25.0 \\
a_{2}=0.00001 \\
a_{3}=0.00001\end{array}$ & $\begin{array}{l}a_{1}=6.027 \\
(\text { error }=0.45 \%) \\
a_{2}=0.0253 \\
(\text { error }=1.2 \%) \\
a_{3}=0.0542 \\
(\text { error }=1.45 \%)\end{array}$ & $\sigma=0.5$ & $\begin{array}{l}\sigma_{a_{1}}=0.2005 \\
\sigma_{a_{2}}=0.0090 \\
\sigma_{a_{3}}=0.0099\end{array}$ & $\begin{array}{l}5.511 \leq a_{1} \leq 6.544 \\
0.0021 \leq a_{2} \leq 0.0484 \\
0.0287 \leq a_{3} \leq 0.0797\end{array}$ \\
\hline
\end{tabular}

Table 6 Statistical analysis results for the estimation of parameters $a_{1}, a_{2}$, and $a_{3}$ in the presence of measurement error by considering the boundary surface $\Gamma_{1}$ as the temperature measurement place.

Test case 2 (Initial guess 2: $a_{1_{\text {initial }_{2}}}=25.0, \quad a_{2_{\text {initial }_{2}}}=0.00001, \quad a_{3_{\text {initial }_{2}}}=0.00001$ ); the boundary surface $\Gamma_{1}$ is the temperature measurement place. The results for the case of no measurement error are shown in Fig. 45 to Fig. 47 and for the case of the measurement error of $\sigma=0.5$ are shown in Fig. 48 to Fig. 50. 


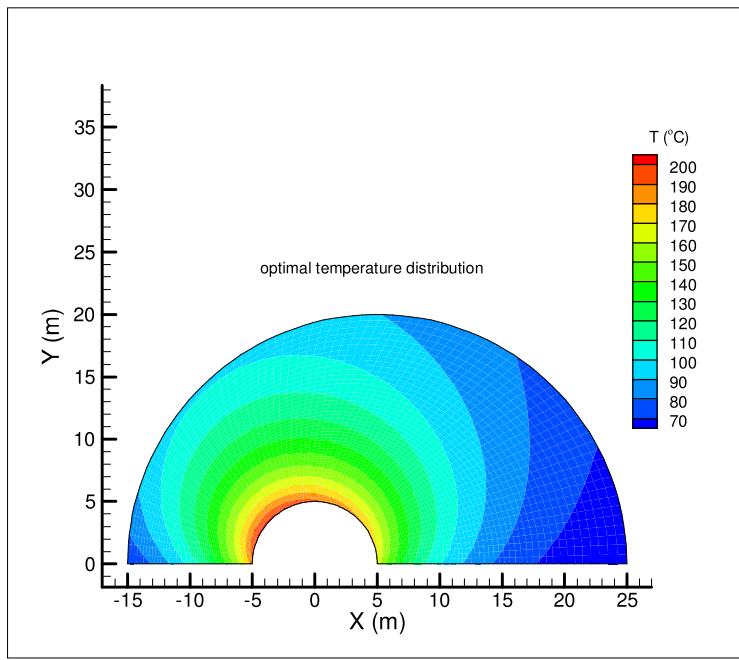

a)

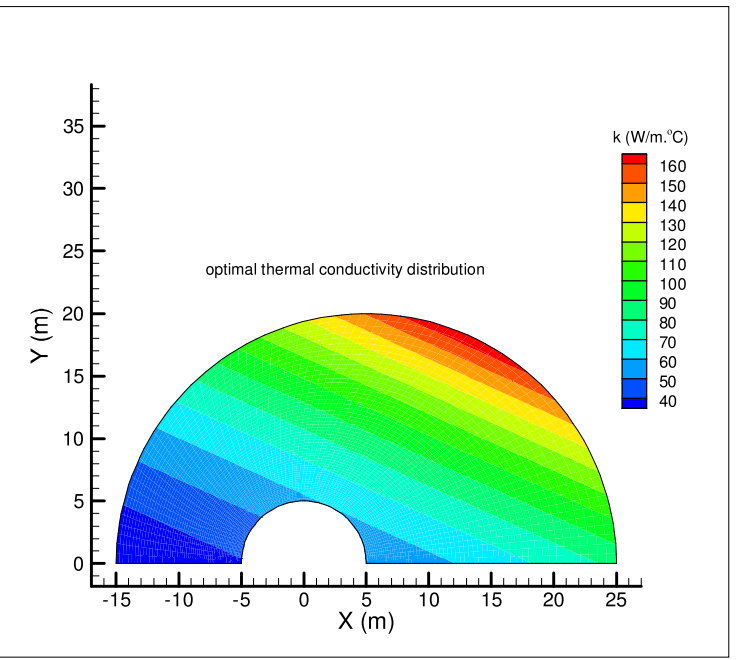

b)

Fig. 45 Optimal temperature distribution (a) and thermal conductivity (b).

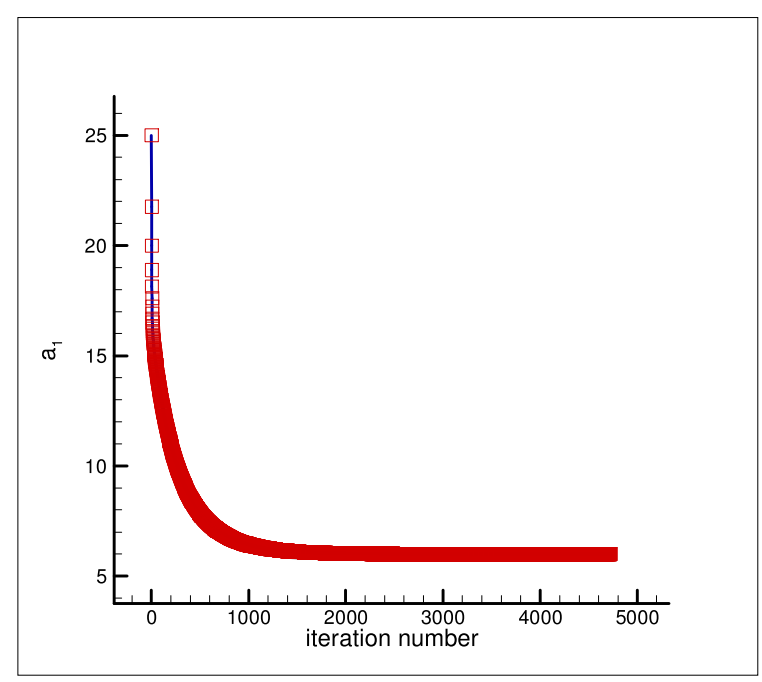

a)

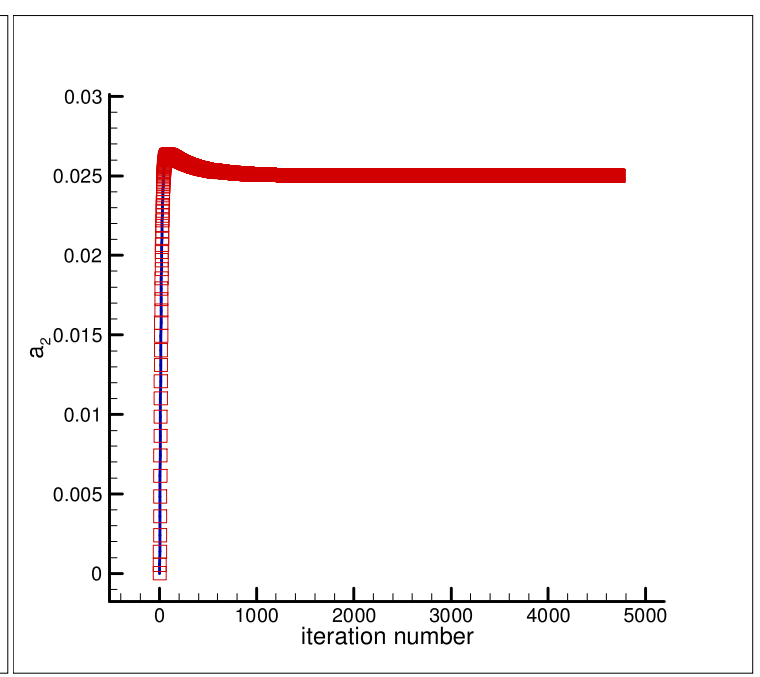

b) 


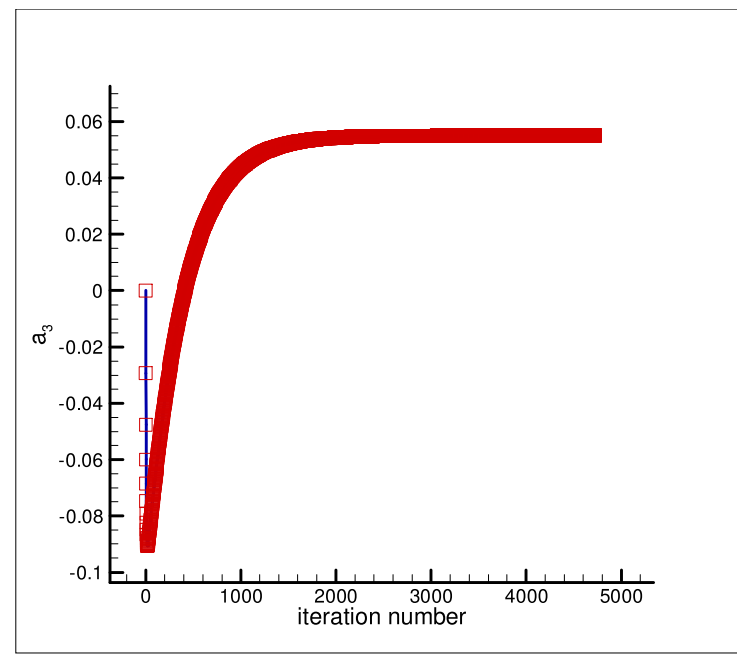

c)

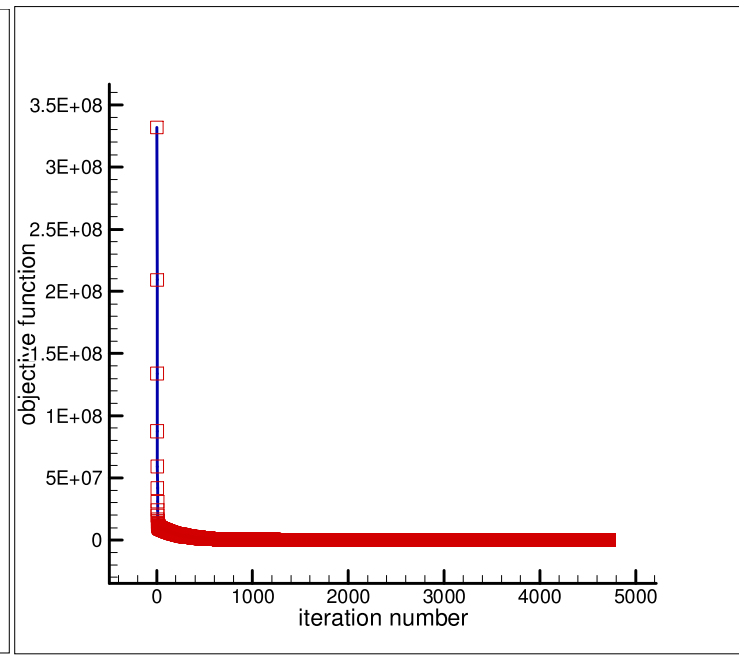

d)

Fig. 46 Estimation of $a_{1}, a_{2}, a_{3}\left(k=a_{1} e^{\left(1+a_{2} x\right)} e^{\left(1+a_{3} y\right)}\right)(\mathrm{a}-\mathrm{c})$ and objective function versus iteration number (d) for initial thermal conductivity $k_{\text {initial }_{2}}=25 e^{\left(1+0.00001 x_{i, j}\right)} e^{\left(1+0.00001 y_{i, j}\right)}\left(\frac{\mathrm{W}}{\mathrm{m} \cdot{ }^{\circ} \mathrm{C}}\right)$ by considering the boundary surface $\Gamma_{1}$ as the temperature measurement place.

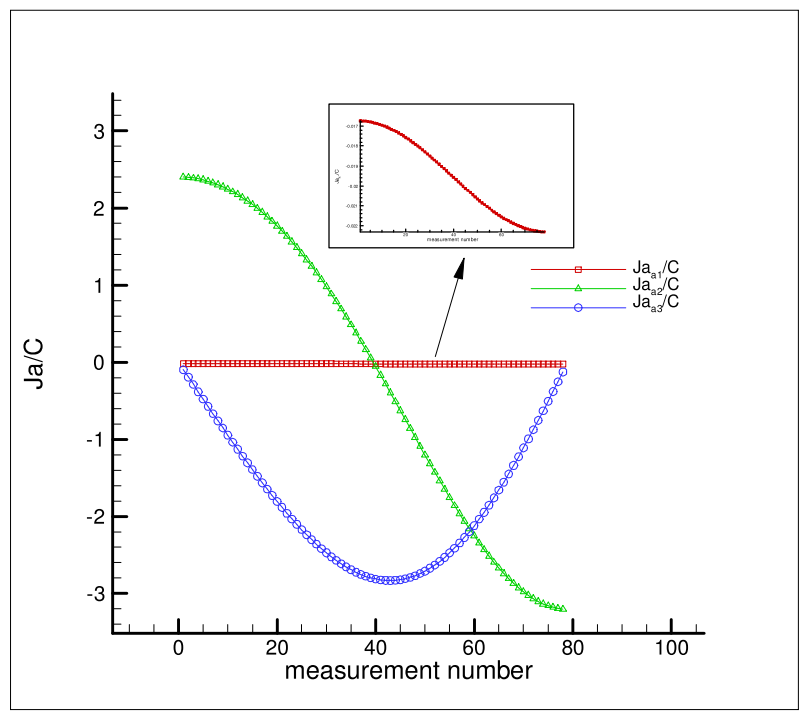

Fig. 47 Sensitivity coefficients given by Eq. (37). 


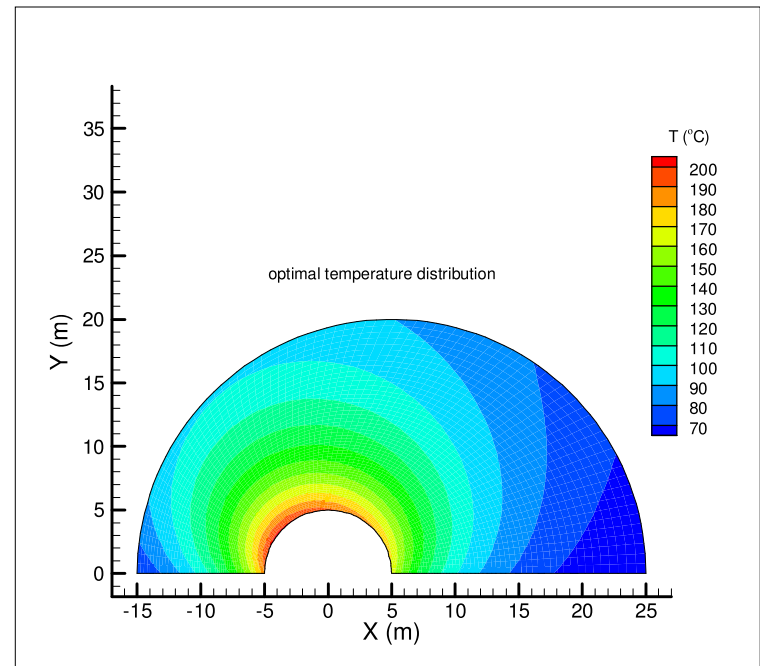

a)

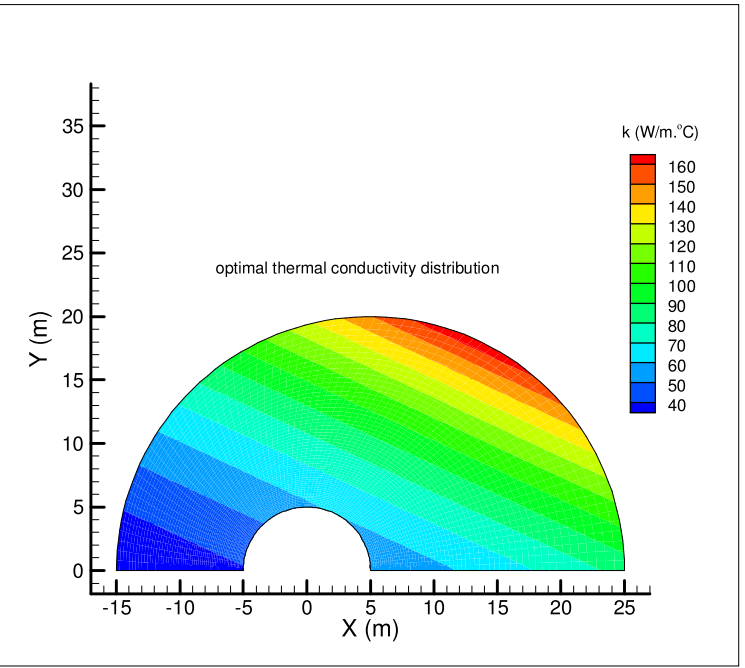

b)

Fig. 48 Optimal temperature distribution (a) and thermal conductivity (b).

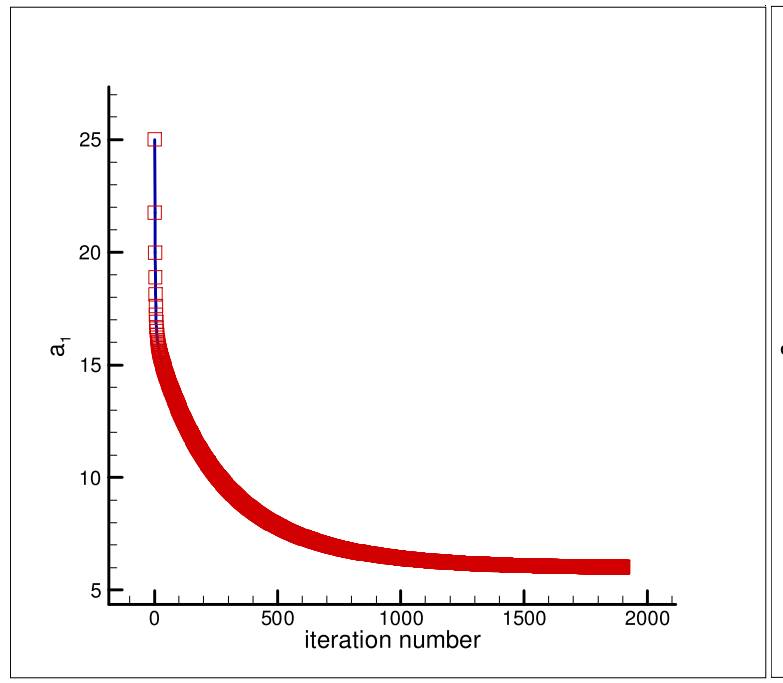

a)

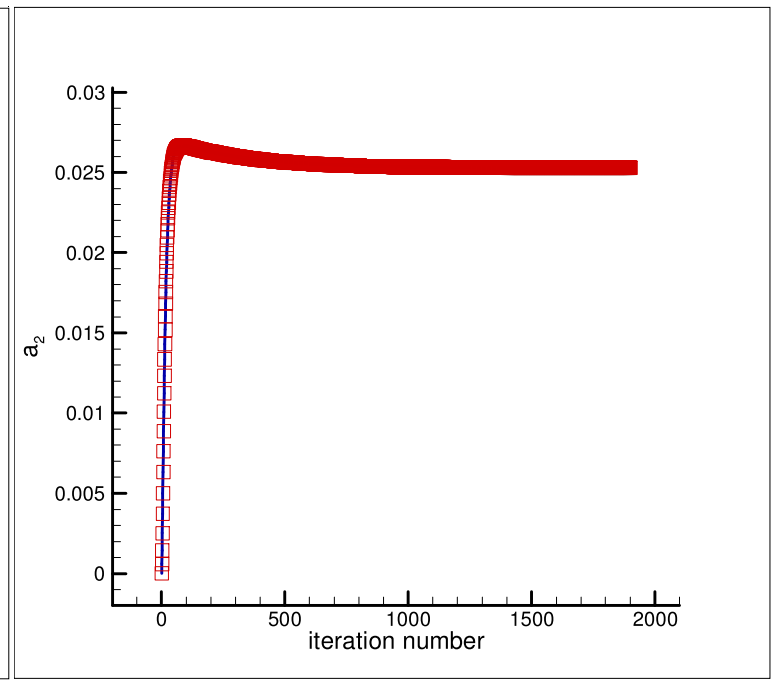

b) 


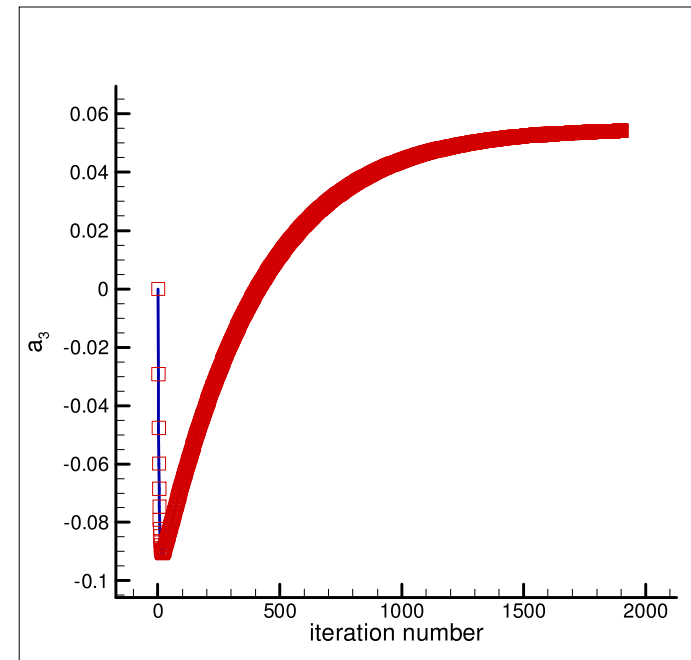

c)

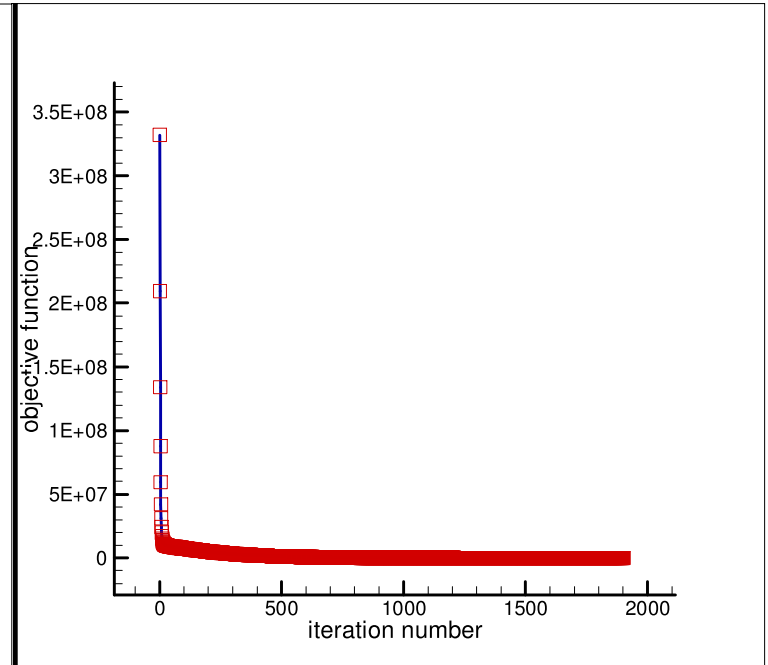

d)

Fig. 49 Estimation of $a_{1}, a_{2}, a_{3}\left(k=a_{1} e^{\left(1+a_{2} x\right)} e^{\left(1+a_{3} y\right)}\right)$ (a-c) and objective function versus iteration number (d) for initial thermal conductivity $k_{\text {initial }_{2}}=25 e^{\left(1+0.00001 x_{i, j}\right)} e^{\left(1+0.00001 y_{i, j}\right)}\left(\frac{\mathrm{W}}{\mathrm{m} .{ }^{\circ} \mathrm{C}}\right)$ by considering the boundary surface $\Gamma_{1}$ as the temperature measurement place and the measurement error $(\sigma=0.5)$.

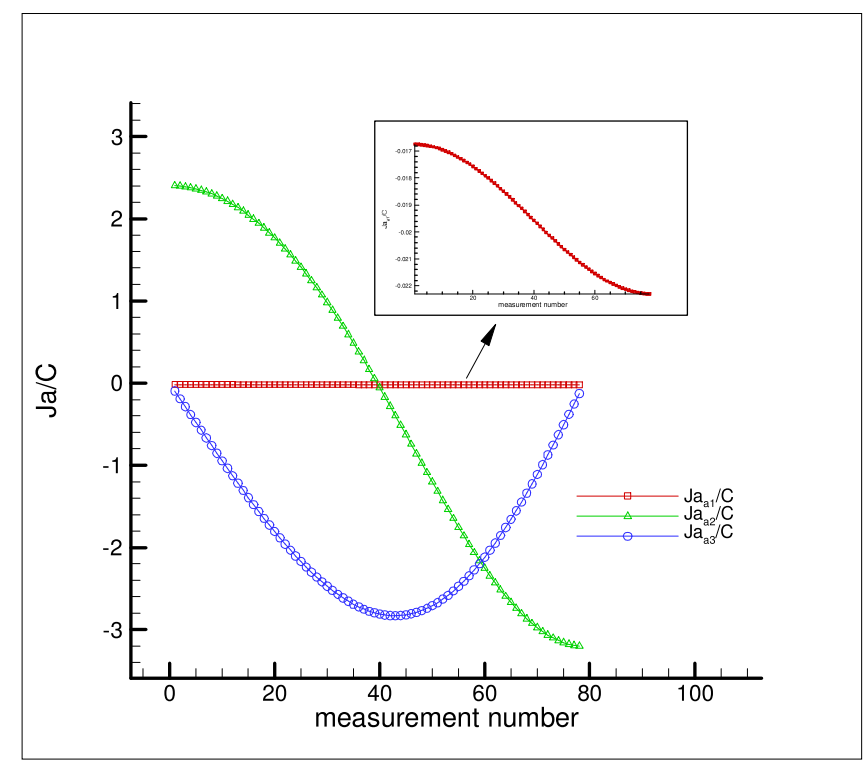

Fig. 50 Sensitivity coefficients given by Eq. (37). 


\section{Conclusion}

This study presented an accurate numerical approach to solve two-dimensional steadystate heat conduction problems in functionally graded materials. The two-dimensional irregular heat-conducting body was transformed into a regular computational domain to perform all computations related to the direct and inverse heat conduction solution. The discretization of the physical domain was performed by the elliptic grid generation and the approximation of the derivatives of the field variable (temperature) at the grid nodes by algebraic ones was performed by using the finite-difference method, a method chosen for the ease of implementation. The proposed numerical method treats the heat conduction equation with the spatially varying thermal conductivity over a general irregular body shape with Dirichlet, Neumann, and Robin boundary conditions as long as the irregular shape can be mapped onto a regular computational domain. Two different types of material gradations are considered for spatially varying thermal conductivity of functionally graded materials, namely, quadratic and exponential material gradations. One of the novelties of this study is to identify the constant parameters in the material gradations accurately by an inverse analysis thereby determining the accurate form of gradation. The novelty of the inverse analysis lies in proposing an accurate and efficient explicit sensitivity analysis scheme with an advantage that it is not involved with an adjoint equation and all the sensitivity coefficients can be computed in only one direct solution, without the need for the solution of the adjoint equation. The conjugate gradient method was used to minimize the objective function and recover the variable thermal conductivity accurately. The accuracy, efficiency, and robustness of the proposed numerical approach were demonstrated through presenting two test cases. Moreover, the results revealed that the retrieved thermal conductivity was not too much affected by introduction of a significant measurement error.

\section{Acknowledgement}

This research was supported by funding from the European Union's Horizon 2020 research and innovation programme under the Marie Skłodowska-Curie grant agreement No 663830 . 


\section{References}

[1] H.S. Shen, Functionally Graded Materials: Nonlinear Analysis of Plates and Shells, CRC Press, 2016.

[2] E.I. E, P. Demetris, G. Cristina, Mechanics Of Functionally Graded Material Structures, World Scientific Publishing Company, 2015.

[3] V. Birman, L.W. Byrd, Modeling and analysis of functionally graded materials and structures, Applied mechanics reviews, 60 (2007) 195-216.

[4] X. Wang, Z. Wang, T. Zeng, S. Cheng, F. Yang, Exact analytical solution for steady-state heat transfer in functionally graded sandwich slabs with convectiveradiative boundary conditions, Composite Structures, 192 (2018) 379-386.

[5] L. Gray, T. Kaplan, J. Richardson, G.H. Paulino, Green's functions and boundary integral analysis for exponentially graded materials: heat conduction, Journal of Applied Mechanics, 70 (2003) 543-549.

[6] A. Sutradhar, G.H. Paulino, The simple boundary element method for transient heat conduction in functionally graded materials, Computer Methods in Applied Mechanics and Engineering, 193 (2004) 4511-4539.

[7] W. Qu, C.-M. Fan, Y. Zhang, Analysis of three-dimensional heat conduction in functionally graded materials by using a hybrid numerical method, International Journal of Heat and Mass Transfer, 145 (2019) 118771.

[8] E. Artyukhin, Reconstruction of the thermal conductivity coefficient from the solution of the nonlinear inverse problem, Journal of Engineering Physics and Thermophysics, 41 (1981) 1054-1058.

[9] O.M. Alifanov, A.P. Tryanin, Determination of the coefficient of internal heat exchange and the effective thermal conductivity of a porous solid on the basis of a nonstationary experiment, Journal of Engineering Physics, 48 (1985) 356-365.

[10] L. Dantas, H. Orlande, A function estimation approach for determining temperature-dependent thermophysical properties, Inverse Problems in Engineering, 3 (1996) 261-279.

[11] T. Jurkowski, Y. Jarny, D. Delaunay, Estimation of thermal conductivity of thermoplastics under moulding conditions: an apparatus and an inverse algorithm, International Journal of Heat and Mass Transfer, 40 (1997) 4169-4181.

[12] C.-y. Yang, A linear inverse model for the temperature-dependent thermal conductivity determination in one-dimensional problems, Applied Mathematical Modelling, 22 (1998) 1-9.

[13] B. Sawaf, M.N. Ozisik, Y. Jarny, An inverse analysis to estimate linearly temperature dependent thermal conductivity components and heat capacity of an 
orthotropic medium, International Journal of Heat and Mass Transfer, 38 (1995) 30053010 .

[14] E. Divo, A.J. Kassab, J.S. Kapat, M.-K. Chyu, Retrieval of multidimensional heat transfer coefficient distributions using an inverse BEM-based regularized algorithm: numerical and experimental results, Engineering Analysis with Boundary Elements, 29 (2005) 150-160.

[15] J. Zhang, M.A. Delichatsios, Determination of the convective heat transfer coefficient in three-dimensional inverse heat conduction problems, Fire Safety Journal, 44 (2009) 681-690.

[16] W.-L. Chen, Y.-C. Yang, H.-L. Lee, Inverse problem in determining convection heat transfer coefficient of an annular fin, Energy Conversion and Management, 48 (2007) 1081-1088.

[17] M. Mierzwiczak, J.A. Kołodziej, The determination temperature-dependent thermal conductivity as inverse steady heat conduction problem, International Journal of Heat and Mass Transfer, 54 (2011) 790-796.

[18] B. Czél, G. Gróf, Inverse identification of temperature-dependent thermal conductivity via genetic algorithm with cost function-based rearrangement of genes, International Journal of Heat and Mass Transfer, 55 (2012) 4254-4263.

[19] C.-H. Huang, Y. Jan-Yuan, An inverse problem in simultaneously measuring temperature-dependent thermal conductivity and heat capacity, International Journal of Heat and Mass Transfer, 38 (1995) 3433-3441.

[20] F. Mohebbi, M. Sellier, Parameter estimation in heat conduction using a twodimensional inverse analysis, International Journal for Computational Methods in Engineering Science and Mechanics, 17 (2016) 274-287.

[21] F. Mohebbi, M. Sellier, T. Rabczuk, Estimation of linearly temperature-dependent thermal conductivity using an inverse analysis, International Journal of Thermal Sciences, 117 (2017) 68-76.

[22] F. Mohebbi, M. Sellier, Identification of space- and temperature-dependent heat transfer coefficient, International Journal of Thermal Sciences, 128 (2018) 28-37.

[23] F. Mohebbi, M. Sellier, Estimation of thermal conductivity, heat transfer coefficient, and heat flux using a three dimensional inverse analysis, International Journal of Thermal Sciences, 99 (2016) 258-270.

[24] P. Tervola, A method to determine the thermal conductivity from measured temperature profiles, International Journal of Heat and Mass Transfer, 32 (1989) 14251430.

[25] S. Kim, A simple direct estimation of temperature-dependent thermal conductivity with kirchhoff transformation, International Communications in Heat and Mass Transfer, 28 (2001) 537-544. 
[26] J.-H. Lin, C.-K. Chen, Y.-T. Yang, Inverse method for estimating thermal conductivity in one-dimensional heat conduction problems, Journal of Thermophysics and Heat Transfer, 15 (2001) 34-41.

[27] S. Chantasiriwan, Steady-state determination of temperature-dependent thermal conductivity, International Communications in Heat and Mass Transfer, 29 (2002) 811819 .

[28] B. Sawaf, M.N. Özisik, Determining the constant thermal conductivities of orthotropic materials by inverse analysis, International Communications in Heat and Mass Transfer, 22 (1995) 201-211.

[29] T.T. Lam, W.K. Yeung, Inverse determination of thermal conductivity for onedimensional problems, Journal of Thermophysics and Heat Transfer, 9 (1995) 335-344.

[30] D. Lesnic, L. Elliott, D.B. Ingham, Identification of the thermal conductivity and heat capacity in unsteady nonlinear heat conduction problems using the boundary element method, Journal of Computational Physics, 126 (1996) 410-420.

[31] B. Konda Reddy, C. Balaji, Estimation of temperature dependent heat transfer coefficient in a vertical rectangular fin using liquid crystal thermography, International Journal of Heat and Mass Transfer, 55 (2012) 3686-3693.

[32] H.R. Orlande, O. Fudym, D. Maillet, R.M. Cotta, Thermal measurements and inverse techniques, CRC Press, 2011.

[33] J. Taler, Determination of local heat transfer coefficient from the solution of the inverse heat conduction problem, Forschung im Ingenieurwesen, 71 (2007) 69-78.

[34] J. Taler, Nonlinear steady-state inverse heat conduction problem with spacevariable boundary conditions, Journal of Heat Transfer (Transactions of the ASME (American Society of Mechanical Engineers), Series C);(United States), 114 (1992).

[35] A.J. Kassab, E. Divo, A generalized boundary integral equation for isotropic heat conduction with spatially varying thermal conductivity, Engineering Analysis with Boundary Elements, 18 (1996) 273-286.

[36] G. Flach, M. Özişik, Inverse heat conduction problem of simultaneously estimating spatially varying thermal conductivity and heat capacity per unit volume, Numerical Heat Transfer, 16 (1989) 249-266.

[37] S.R. Reddy, G.S. Dulikravich, S.J. Zeidi, Non-destructive estimation of spatially varying thermal conductivity in $3 \mathrm{D}$ objects using boundary thermal measurements, International Journal of Thermal Sciences, 118 (2017) 488-496.

[38] M.I.P. Hidayat, B.A. Wahjoedi, S. Parman, P.S.M. Yusoff, Meshless local B-splineFD method and its application for $2 \mathrm{D}$ heat conduction problems with spatially varying thermal conductivity, Applied Mathematics and Computation, 242 (2014) 236-254.

[39] J.V. Beck, Surface heat flux determination using an integral method, Nuclear Engineering and Design, 7 (1968) 170-178. 
[40] C.H. Huang, S.P. Wang, A three-dimensional inverse heat conduction problem in estimating surface heat flux by conjugate gradient method, International Journal of Heat and Mass transfer, 42 (1999) 3387-3403.

[41] F. Mohebbi, B. Evans, A. Shaw, M. Sellier, An inverse analysis for determination of space-dependent heat flux in heat conduction problems in the presence of variable thermal conductivity, International Journal for Computational Methods in Engineering Science and Mechanics, 20 (2019) 229-241.

[42] F. Mohebbi, B. Evans, Simultaneous estimation of heat flux and heat transfer coefficient in irregular geometries made of functionally graded materials, International Journal of Thermofluids, 1-2 (2020) 100009.

[43] F. Mohebbi, Function Estimation in Inverse Heat Transfer Problems Based on Parameter Estimation Approach, Energies, 13 (2020) 4410.

[44] C.K. Hsieh, A.J. Kassab, A general method for the solution of inverse heat conduction problems with partially unknown system geometries, International Journal of Heat and Mass Transfer, 29 (1986) 47-58.

[45] F. Mohebbi, M. Sellier, Optimal shape design in heat transfer based on body-fitted grid generation, International Journal for Computational Methods in Engineering Science and Mechanics, 14 (2013) 227-243.

[46] F. Mohebbi, M. Sellier, Three-dimensional optimal shape design in heat transfer based on body-fitted grid generation, International Journal for Computational Methods in Engineering Science and Mechanics, 14 (2013) 473-490.

[47] F. Mohebbi, M. Sellier, T. Rabczuk, Inverse problem of simultaneously estimating the thermal conductivity and boundary shape, International Journal for Computational Methods in Engineering Science and Mechanics, (2017) 1-16.

[48] S.M.H. Sarvari, Optimal Geometry Design of Radiative Enclosures Using the Genetic Algorithm, Numerical Heat Transfer, Part A: Applications, 52 (2007) 127-143.

[49] O.M. Alifanov, Inverse heat transfer problems, Springer-Verlag, 1994.

[50] M. Özisik, H. Orlande, Inverse heat transfer: fundamentals and applications, Taylor \& Francis, 2000.

[51] J.V. Beck, B. Blackwell, C.R.S. Clair, Inverse Heat Conduction: Ill-Posed Problems, Wiley, 1985.

[52] L. Marin, Numerical solution of the Cauchy problem for steady-state heat transfer in two-dimensional functionally graded materials, International Journal of Solids and Structures, 42 (2005) 4338-4351.

[53] W.-L. Chen, H.-M. Chou, Y.-C. Yang, An inverse problem in estimating the spacedependent thermal conductivity of a functionally graded hollow cylinder, Composites Part B: Engineering, 50 (2013) 112-119. 
[54] G.S. Dulikravich, S.R. Reddy, M.A. Pasqualette, M.J. Colaço, H.R. Orlande, J. Coverston, Inverse determination of spatially varying material coefficients in solid objects, Journal of Inverse and Ill-posed Problems, 24 (2016) 181-194.

[55] H.-L. Lee, W.-J. Chang, S.-H. Sun, Y.-C. Yang, Estimation of temperature distributions and thermal stresses in a functionally graded hollow cylinder simultaneously subjected to inner-and-outer boundary heat fluxes, Composites Part B: Engineering, 43 (2012) 786-792.

[56] M.G. Haghighi, P. Malekzadeh, M. Afshari, Inverse estimation of heat flux and pressure in functionally graded cylinders with finite length, Composite Structures, 121 (2015) 1-15.

[57] F. Mohebbi, Optimal shape design based on body-fitted grid generation, University of Canterbury, (2014).

[58] M.N. Özişik, H.R.B. Orlande, M.J. Colaço, R.M. Cotta, Finite Difference Methods in Heat Transfer, CRC Press, 2017.

[59] E. Polak, G. Ribiere, Note sur la convergence de méthodes de directions conjuguées, Revue Française d'Informatique et de Recherche Opérationnelle, 16 (1969) 35-43.

[60] J.V. Beck, K.J. Arnold, Parameter estimation in engineering and science, Wiley, 1977. 